The Importance of How the Pieces Fit Together: The Microwave Spectrum and Molecular

\title{
Structure of 2-Chloro-1,1-Difluoroethylene-Acetylene
}

\author{
Helen O. Leung ${ }^{*}$ and Mark D. Marshall* \\ Department of Chemistry, Amherst College, P.O. Box 5000, Amherst, MA 01002-5000 \\ Supporting Information
}

Address for correspondence: Prof. Mark D. Marshall

Department of Chemistry

Amherst College

P.O. Box 5000

Amherst, MA 01002-5000

Telephone: (413) 542-2006

Fax: (413) 542-2735

E-mail: mdmarshall@amherst.edu

*Corresponding authors. Fax: +1-413-542-2735; e-mail addresses: hleung@amherst.edu (H.O. Leung), mdmarshall@amherst.edu (M.D. Marshall).

The authors declare no competing financial interest. 
Table S1 presents the atomic coordinates in the appropriate principal inertial axis system for the four theoretical structures presented in Figure 1 of the manuscript.

Tables S2, S3, S4, and S5 contain the quantum number assignments, observed transition frequencies (in MHz), and the residuals (obs. - calc., also in MHz) from the least squares fits.

The quantum numbers are $J, K_{a}$, and $K_{c}$, the usual asymmetric top rotational quantum numbers for the initial and final levels of the transition. $F=J+I$ is the total angular momentum, where $I$ $=3 / 2$ is the nuclear spin angular momentum of the chlorine nucleus.

\section{The complete reference for Gaussian 16 (reference 22) is given here.}

Frisch, M. J.; Trucks, G. W.; Schlegel, H. B.; Scuseria, G. E.; Robb, M. A.; Cheeseman, J. R.;

Scalmani, G.; Barone, V.; Petersson, G. A.; Nakatsuji, H.; Li, X.; Caricato, M.; Marenich, A. V.;

Bloino, J.; Janesko, B. G.; Gomperts, R.; Mennucci, B.; Hratchian, H. P.; Ortiz, J. V.; Izmaylov, A. F.; Sonnenberg, J. L.; Williams-Young, F.; Ding, F.; Lipparini, F.; Egidi, F.; Goings, J.; Peng, B.; Petrone, A.; Henderson, T.; Ranasinghe, D.; Zakrzewski, V. G.; Gao, J.; Rega, N.; Zheng, G.; Liang, W.; Hada, M.; Ehara, M.; Toyota, K.; Fukuda, R.; Hasegawa, J.; Ishida, M.; Nakajima, T.; Honda, Y.; Kitao, O.; Nakai, H.; Vreven, T.; Throssell, K.; Montgomery Jr., J. A.; Peralta, J. E.; Ogliaro, F.; Bearpark, M. J.; Heyd, J. J.; Brothers, E. N.; Kudin, K. N.; Staroverov, V. N.; Keith, T. A.; Kobayashi, R.; Normand, J.; Raghavachari, K.; Rendell, A. P.; Burant, J. C.; Iyengar, S. S.; Tomasi, J.; Cossi, M.; Millam, J. M.; Klene, M.; Adamo, C.; Cammi, R.; Ochterski, J. W.; Martin, R. L.; Morokuma, K.; Farkas, O.; Foresman, J. B.; Fox, D. J. Gaussian 16, Revision A.03; Wallingford, CT, 2016. 
Table S1: Principal inertial axis system coordinates for the 4 theoretical structures in Fig. 1.

\section{No BSSE Correction}

\begin{tabular}{cccccc} 
& Structure (a) & & \multicolumn{3}{c}{ Structure (b) } \\
$a$ & $b$ & $c$ & $a$ & $b$ & $c$
\end{tabular}

$\begin{array}{lcccccc}\mathrm{CF}_{2} \mathrm{CHCl} \text { subunit } & & & & & \\ \mathrm{C} & -0.1412 & -0.0684 & 0.0000 & -0.8505 & -0.2783 & -0.7621 \\ \mathrm{H} & 0.7843 & -0.6301 & 0.0000 & -1.0079 & -0.3128 & -1.8327 \\ \mathrm{Cl} & 0.1853 & 1.6316 & 0.0000 & -1.8252 & 0.9620 & -0.0491 \\ \mathrm{C} & -1.3622 & -0.5232 & 0.0000 & -0.0639 & -1.0218 & -0.0369 \\ \mathrm{~F} & -2.4633 & 0.2039 & 0.0000 & 0.0949 & -0.9420 & 1.2706 \\ \mathrm{~F} & -1.6595 & -1.8103 & 0.0000 & 0.6973 & -1.9791 & -0.5362 \\ \mathrm{HCCH} & \text { subunit } & & & & & \\ \mathrm{H} & 3.2047 & 0.9243 & 0.0000 & 3.6934 & 1.0493 & -0.9000 \\ \mathrm{C} & 3.3448 & -0.1239 & 0.0000 & 2.8292 & 1.3054 & -0.3468 \\ \mathrm{C} & 3.5049 & -1.3216 & 0.0000 & 1.8419 & 1.5981 & 0.2853 \\ \mathrm{H} & 3.6449 & -2.3698 & 0.0000 & 0.9778 & 1.8543 & 0.8386\end{array}$

\begin{tabular}{|c|c|c|c|c|c|}
\hline \multicolumn{3}{|c|}{ Structure (c) } & \multicolumn{3}{|c|}{ Structure (d) } \\
\hline$a$ & $b$ & $c$ & $a$ & $b$ & $c$ \\
\hline \multicolumn{6}{|l|}{$\mathrm{CF}_{2} \mathrm{CHCl}$ subunit } \\
\hline 0.2982 & 0.3920 & 0.0001 & 0.9823 & 0.8765 & 0.0001 \\
\hline-0.6732 & 0.8699 & 0.0003 & 1.0953 & 1.9532 & 0.0002 \\
\hline 1.5555 & 1.5818 & 0.0000 & -0.6944 & 0.4462 & 0.0000 \\
\hline 0.5716 & -0.8819 & 0.0001 & 1.9147 & -0.0335 & 0.0000 \\
\hline 1.7769 & -1.4190 & -0.0001 & 1.7272 & -1.3397 & -0.0001 \\
\hline-0.3534 & -1.8250 & 0.0002 & 3.2056 & 0.2469 & 0.0000 \\
\hline \multicolumn{6}{|l|}{$\mathrm{HCCH}$ subunit } \\
\hline-4.2061 & 1.9419 & 0.0004 & -4.0478 & -0.2657 & -1.6617 \\
\hline-3.7602 & 0.9829 & 0.0001 & -4.0476 & -0.2661 & -0.6042 \\
\hline-3.2508 & -0.1128 & -0.0003 & -4.0475 & -0.2665 & 0.6041 \\
\hline-2.8050 & -1.0717 & -0.0005 & -4.0474 & -0.2668 & 1.6617 \\
\hline
\end{tabular}

\section{With BSSE Correction}

\begin{tabular}{|c|c|c|c|c|c|c|}
\hline & \multicolumn{3}{|c|}{ Structure (a) } & \multicolumn{3}{|c|}{ Structure (b) } \\
\hline & $a$ & $b$ & $c$ & $a$ & $b$ & $c$ \\
\hline \multicolumn{7}{|c|}{$\mathrm{CF}_{2} \mathrm{CHCl}$ subunit } \\
\hline $\mathrm{C}$ & -0.1599 & -0.0672 & 0.0000 & -0.8835 & -0.1661 & -0.7680 \\
\hline $\mathrm{H}$ & 0.7705 & -0.6208 & 0.0001 & -1.0213 & -0.1570 & -1.8418 \\
\hline $\mathrm{Cl}$ & 0.1518 & 1.6356 & 0.0000 & -1.6647 & 1.2007 & -0.0482 \\
\hline $\mathrm{C}$ & -1.3768 & -0.5326 & 0.0000 & -0.2401 & -1.0372 & -0.0436 \\
\hline $\mathrm{F}$ & -2.4843 & 0.1848 & 0.0000 & -0.0988 & -1.0060 & 1.2680 \\
\hline $\mathrm{F}$ & -1.6629 & -1.8223 & 0.0000 & 0.3706 & -2.0945 & -0.5480 \\
\hline \multicolumn{7}{|c|}{ HCCH subunit } \\
\hline $\mathrm{H}$ & 3.2690 & 0.9432 & -0.0002 & 3.9039 & 0.6214 & -0.8550 \\
\hline $\mathrm{C}$ & 3.4182 & -0.1038 & -0.0001 & 3.0721 & 0.9969 & -0.3206 \\
\hline
\end{tabular}


Table S1: Principal inertial axis system coordinates for the 4 theoretical structures in Fig. 1.

\begin{tabular}{|c|c|c|c|c|c|c|}
\hline $\mathrm{C}$ & 3.5887 & -1.3000 & 0.0000 & 2.1218 & 1.4259 & 0.2900 \\
\hline $\mathrm{H}$ & 3.7380 & -2.3470 & 0.0002 & 1.2900 & 1.8013 & 0.8244 \\
\hline & \multicolumn{3}{|c|}{ Structure (c) } & \multicolumn{3}{|c|}{ Structure (d) } \\
\hline & $a$ & $b$ & $c$ & $a$ & $b$ & $c$ \\
\hline \multicolumn{7}{|c|}{$\mathrm{CF}_{2} \mathrm{CHCl}$ subunit } \\
\hline $\mathrm{C}$ & 0.3215 & 0.3961 & -0.0021 & 1.0114 & 0.8774 & 0.0084 \\
\hline $\mathrm{H}$ & -0.6476 & 0.8788 & -0.0045 & 1.1312 & 1.9534 & 0.0199 \\
\hline $\mathrm{Cl}$ & 1.5847 & 1.5796 & 0.0003 & -0.6680 & 0.4579 & 0.0024 \\
\hline $\mathrm{C}$ & 0.5887 & -0.8791 & -0.0009 & 1.9380 & -0.0385 & -0.0004 \\
\hline $\mathrm{F}$ & 1.7913 & -1.4222 & 0.0022 & 1.7422 & -1.3433 & -0.0144 \\
\hline $\mathrm{F}$ & -0.3410 & -1.8177 & -0.0026 & 3.2307 & 0.2338 & 0.0037 \\
\hline \multicolumn{7}{|c|}{ HCCH subunit } \\
\hline $\mathrm{H}$ & -4.2770 & 1.9417 & -0.0064 & -4.1138 & -0.3317 & 1.6604 \\
\hline $\mathrm{C}$ & -3.8359 & 0.9805 & -0.0015 & -4.1291 & -0.2911 & 0.6038 \\
\hline $\mathrm{C}$ & -3.3319 & -0.1176 & 0.0042 & -4.1465 & -0.2447 & -0.6035 \\
\hline $\mathrm{H}$ & -2.8908 & -1.0788 & 0.0091 & -4.1618 & -0.2040 & -1.6602 \\
\hline
\end{tabular}


Table S2: Observed Transition Frequencies (in $\mathrm{MHz}$ ) for $\mathrm{CF}_{2} \mathrm{CH}^{35} \mathrm{Cl}-\mathrm{HCCH}$

\begin{tabular}{|c|c|c|c|c|c|c|c|c|c|}
\hline$J^{\prime}$ & $K_{a}^{\prime}$ & $K_{c}{ }^{\prime}$ & $F^{\prime}$ & $J^{\prime \prime}$ & $K_{a}^{\prime \prime}$ & $K_{c}^{\prime \prime}$ & $F^{\prime \prime}$ & Observed & Obs - Calc \\
\hline 3 & 2 & 2 & 4.5 & 2 & 2 & 1 & 3.5 & 5126.4133 & -0.0004 \\
\hline 3 & 2 & 2 & 2.5 & 2 & 2 & 1 & 1.5 & 5129.8194 & -0.0003 \\
\hline 3 & 2 & 2 & 3.5 & 2 & 2 & 1 & 2.5 & 5135.1422 & 0.0007 \\
\hline 3 & 2 & 1 & 4.5 & 2 & 2 & 0 & 3.5 & 5239.6599 & -0.0001 \\
\hline 3 & 2 & 1 & 2.5 & 2 & 2 & 0 & 1.5 & 5244.3220 & 0.0013 \\
\hline 3 & 2 & 1 & 3.5 & 2 & 2 & 0 & 2.5 & 5251.6375 & -0.0006 \\
\hline 4 & 0 & 4 & 2.5 & 3 & 1 & 3 & 1.5 & 5415.0010 & 0.0005 \\
\hline 4 & 0 & 4 & 5.5 & 3 & 1 & 3 & 4.5 & 5418.8634 & 0.0003 \\
\hline 4 & 0 & 4 & 3.5 & 3 & 1 & 3 & 2.5 & 5423.2159 & -0.0004 \\
\hline 4 & 0 & 4 & 4.5 & 3 & 1 & 3 & 3.5 & 5426.8490 & -0.0008 \\
\hline 3 & 1 & 2 & 1.5 & 2 & 1 & 1 & 1.5 & 5493.5702 & 0.0012 \\
\hline 3 & 1 & 2 & 2.5 & 2 & 1 & 1 & 2.5 & 5500.2071 & -0.0004 \\
\hline 3 & 1 & 2 & 4.5 & 2 & 1 & 1 & 3.5 & 5507.8572 & -0.0002 \\
\hline 3 & 1 & 2 & 3.5 & 2 & 1 & 1 & 2.5 & 5509.4364 & -0.0001 \\
\hline 3 & 1 & 2 & 1.5 & 2 & 1 & 1 & 0.5 & 5511.6448 & 0.0011 \\
\hline 3 & 1 & 2 & 2.5 & 2 & 1 & 1 & 1.5 & 5513.2032 & 0.0003 \\
\hline 3 & 1 & 2 & 3.5 & 2 & 1 & 1 & 3.5 & 5527.5903 & -0.0009 \\
\hline 6 & 1 & 5 & 4.5 & 6 & 0 & 6 & 4.5 & 5773.9292 & -0.0011 \\
\hline 6 & 1 & 5 & 7.5 & 6 & 0 & 6 & 7.5 & 5779.1690 & -0.0019 \\
\hline 6 & 1 & 5 & 5.5 & 6 & 0 & 6 & 5.5 & 5795.7457 & -0.0011 \\
\hline 6 & 1 & 5 & 6.5 & 6 & 0 & 6 & 6.5 & 5800.8487 & -0.0025 \\
\hline 2 & 2 & 1 & 1.5 & 2 & 1 & 2 & 0.5 & 5839.9179 & -0.0015 \\
\hline 2 & 2 & 1 & 2.5 & 2 & 1 & 2 & 3.5 & 5840.0143 & -0.0016 \\
\hline 2 & 2 & 1 & 2.5 & 2 & 1 & 2 & 1.5 & 5842.5185 & -0.0005 \\
\hline 2 & 2 & 1 & 2.5 & 2 & 1 & 2 & 2.5 & 5849.0397 & -0.0043 \\
\hline 2 & 2 & 1 & 3.5 & 2 & 1 & 2 & 3.5 & 5849.0632 & 0.0023 \\
\hline 2 & 2 & 1 & 1.5 & 2 & 1 & 2 & 2.5 & 5855.5416 & -0.0010 \\
\hline 2 & 2 & 1 & 0.5 & 2 & 1 & 2 & 1.5 & 5858.0545 & -0.0041 \\
\hline 2 & 2 & 1 & 3.5 & 2 & 1 & 2 & 2.5 & 5858.0935 & 0.0045 \\
\hline 3 & 1 & 3 & 3.5 & 2 & 0 & 2 & 2.5 & 6146.6230 & 0.0001 \\
\hline 3 & 1 & 3 & 2.5 & 2 & 0 & 2 & 2.5 & 6150.0956 & -0.0012 \\
\hline 3 & 1 & 3 & 3.5 & 2 & 0 & 2 & 3.5 & 6153.6278 & -0.0003 \\
\hline 3 & 1 & 3 & 2.5 & 2 & 0 & 2 & 1.5 & 6155.0553 & -0.0006 \\
\hline 3 & 1 & 3 & 4.5 & 2 & 0 & 2 & 3.5 & 6161.0108 & 0.0008 \\
\hline 3 & 1 & 3 & 1.5 & 2 & 0 & 2 & 1.5 & 6162.4851 & -0.0025 \\
\hline 3 & 1 & 3 & 1.5 & 2 & 0 & 2 & 0.5 & 6169.5281 & -0.0007 \\
\hline 4 & 1 & 4 & 4.5 & 3 & 1 & 3 & 4.5 & 6244.0470 & -0.0011 \\
\hline 4 & 1 & 4 & 2.5 & 3 & 1 & 3 & 1.5 & 6249.6538 & 0.0007 \\
\hline 4 & 1 & 4 & 3.5 & 3 & 1 & 3 & 2.5 & 6250.8232 & 0.0000 \\
\hline 4 & 1 & 4 & 4.5 & 3 & 1 & 3 & 3.5 & 6251.4298 & -0.0002 \\
\hline 4 & 1 & 4 & 5.5 & 3 & 1 & 3 & 4.5 & 6251.7638 & -0.0010 \\
\hline 4 & 1 & 4 & 3.5 & 3 & 1 & 3 & 3.5 & 6254.2955 & -0.0015 \\
\hline 4 & 1 & 4 & 2.5 & 3 & 1 & 3 & 2.5 & 6257.0838 & -0.0009 \\
\hline
\end{tabular}


Table S2: Observed Transition Frequencies (in $\mathrm{MHz}$ ) for $\mathrm{CF}_{2} \mathrm{CH}^{35} \mathrm{Cl}-\mathrm{HCCH}$

\begin{tabular}{|c|c|c|c|c|c|c|c|c|c|}
\hline$J^{\prime}$ & $K_{a}^{\prime}$ & $K_{c}{ }^{\prime}$ & $F^{\prime}$ & $J^{\prime \prime}$ & $K_{a}^{\prime \prime}$ & $K_{c}^{\prime \prime}$ & $F^{\prime \prime}$ & Observed & Obs - Calc \\
\hline 3 & 2 & 2 & 1.5 & 3 & 1 & 3 & 1.5 & 6260.5960 & 0.0002 \\
\hline 3 & 2 & 2 & 2.5 & 3 & 1 & 3 & 1.5 & 6261.8930 & 0.0000 \\
\hline 3 & 2 & 2 & 3.5 & 3 & 1 & 3 & 4.5 & 6264.2360 & -0.0038 \\
\hline 3 & 2 & 2 & 4.5 & 3 & 1 & 3 & 4.5 & 6264.5573 & 0.0003 \\
\hline 3 & 2 & 2 & 1.5 & 3 & 1 & 3 & 2.5 & 6268.0263 & -0.0011 \\
\hline 3 & 2 & 2 & 3.5 & 3 & 1 & 3 & 2.5 & 6268.1482 & 0.0004 \\
\hline 3 & 2 & 2 & 2.5 & 3 & 1 & 3 & 2.5 & 6269.3235 & -0.0012 \\
\hline 3 & 2 & 2 & 3.5 & 3 & 1 & 3 & 3.5 & 6271.6209 & -0.0008 \\
\hline 3 & 2 & 2 & 2.5 & 3 & 1 & 3 & 3.5 & 6272.7983 & -0.0002 \\
\hline 4 & 0 & 4 & 3.5 & 3 & 0 & 3 & 3.5 & 6561.8587 & -0.0006 \\
\hline 4 & 0 & 4 & 4.5 & 3 & 0 & 3 & 3.5 & 6562.0185 & -0.0005 \\
\hline 4 & 0 & 4 & 2.5 & 3 & 0 & 3 & 2.5 & 6562.9458 & 0.0014 \\
\hline 4 & 0 & 4 & 3.5 & 3 & 0 & 3 & 2.5 & 6563.7282 & -0.0003 \\
\hline 4 & 0 & 4 & 5.5 & 3 & 0 & 3 & 4.5 & 6565.4781 & -0.0004 \\
\hline 4 & 0 & 4 & 4.5 & 3 & 0 & 3 & 4.5 & 6566.0848 & 0.0014 \\
\hline 4 & 0 & 4 & 2.5 & 3 & 0 & 3 & 1.5 & 6567.0595 & 0.0001 \\
\hline 4 & 2 & 3 & 2.5 & 3 & 2 & 2 & 2.5 & 6811.5730 & -0.0019 \\
\hline 4 & 2 & 3 & 2.5 & 3 & 2 & 2 & 1.5 & 6812.8733 & 0.0013 \\
\hline 4 & 2 & 3 & 5.5 & 3 & 2 & 2 & 4.5 & 6813.2922 & 0.0002 \\
\hline 4 & 2 & 3 & 3.5 & 3 & 2 & 2 & 2.5 & 6814.3855 & 0.0002 \\
\hline 4 & 2 & 3 & 3.5 & 3 & 2 & 2 & 3.5 & 6815.5621 & 0.0000 \\
\hline 4 & 2 & 3 & 4.5 & 3 & 2 & 2 & 4.5 & 6816.6264 & -0.0012 \\
\hline 4 & 2 & 3 & 4.5 & 3 & 2 & 2 & 3.5 & 6816.9452 & 0.0003 \\
\hline 4 & 2 & 3 & 2.5 & 4 & 1 & 4 & 2.5 & 6823.8133 & -0.0014 \\
\hline 4 & 2 & 3 & 5.5 & 4 & 1 & 4 & 5.5 & 6826.0840 & -0.0003 \\
\hline 4 & 2 & 3 & 3.5 & 4 & 1 & 4 & 3.5 & 6832.8858 & -0.0009 \\
\hline 4 & 2 & 3 & 4.5 & 4 & 1 & 4 & 4.5 & 6837.1356 & -0.0010 \\
\hline 4 & 3 & 2 & 2.5 & 3 & 3 & 1 & 1.5 & 6883.5570 & 0.0019 \\
\hline 4 & 3 & 2 & 4.5 & 3 & 3 & 1 & 4.5 & 6886.2032 & -0.0005 \\
\hline 4 & 3 & 2 & 5.5 & 3 & 3 & 1 & 4.5 & 6887.9488 & -0.0012 \\
\hline 4 & 3 & 2 & 3.5 & 3 & 3 & 1 & 2.5 & 6892.5387 & 0.0008 \\
\hline 4 & 3 & 2 & 2.5 & 3 & 3 & 1 & 2.5 & 6894.2925 & -0.0002 \\
\hline 4 & 3 & 1 & 2.5 & 3 & 3 & 0 & 1.5 & 6895.3465 & -0.0001 \\
\hline 4 & 3 & 2 & 4.5 & 3 & 3 & 1 & 3.5 & 6896.9180 & 0.0010 \\
\hline 4 & 3 & 2 & 3.5 & 3 & 3 & 1 & 3.5 & 6897.5437 & -0.0007 \\
\hline 4 & 3 & 1 & 4.5 & 3 & 3 & 0 & 4.5 & 6898.5453 & -0.0012 \\
\hline 4 & 3 & 1 & 5.5 & 3 & 3 & 0 & 4.5 & 6899.8006 & -0.0004 \\
\hline 4 & 3 & 1 & 3.5 & 3 & 3 & 0 & 2.5 & 6904.5912 & 0.0014 \\
\hline 4 & 3 & 1 & 2.5 & 3 & 3 & 0 & 2.5 & 6905.9780 & 0.0004 \\
\hline 4 & 3 & 1 & 4.5 & 3 & 3 & 0 & 3.5 & 6909.1514 & 0.0009 \\
\hline 4 & 3 & 1 & 3.5 & 3 & 3 & 0 & 3.5 & 6909.5455 & -0.0004 \\
\hline 4 & 2 & 2 & 2.5 & 3 & 2 & 1 & 2.5 & 7079.4015 & 0.0027 \\
\hline 4 & 2 & 2 & 2.5 & 3 & 2 & 1 & 1.5 & 7084.3243 & 0.0013 \\
\hline
\end{tabular}


Table S2: Observed Transition Frequencies (in $\mathrm{MHz}$ ) for $\mathrm{CF}_{2} \mathrm{CH}^{35} \mathrm{Cl}-\mathrm{HCCH}$

\begin{tabular}{|c|c|c|c|c|c|c|c|c|c|}
\hline$J^{\prime}$ & $K_{a}^{\prime}$ & $K_{c}{ }^{\prime}$ & $F^{\prime}$ & $J^{\prime \prime}$ & $K_{a}^{\prime \prime}$ & $K_{c}^{\prime \prime}$ & $F^{\prime \prime}$ & Observed & Obs - Calc \\
\hline 4 & 2 & 2 & 5.5 & 3 & 2 & 1 & 4.5 & 7086.0648 & -0.0001 \\
\hline 4 & 2 & 2 & 3.5 & 3 & 2 & 1 & 3.5 & 7088.5342 & 0.0002 \\
\hline 4 & 2 & 2 & 3.5 & 3 & 2 & 1 & 2.5 & 7090.8850 & -0.0001 \\
\hline 4 & 2 & 2 & 4.5 & 3 & 2 & 1 & 3.5 & 7092.5903 & 0.0002 \\
\hline 4 & 2 & 2 & 4.5 & 3 & 2 & 1 & 4.5 & 7097.6012 & -0.0018 \\
\hline 8 & 3 & 5 & 8.5 & 8 & 2 & 6 & 8.5 & 7142.4191 & 0.0012 \\
\hline 8 & 3 & 5 & 7.5 & 8 & 2 & 6 & 7.5 & 7143.5240 & -0.0002 \\
\hline 8 & 3 & 5 & 9.5 & 8 & 2 & 6 & 9.5 & 7148.6155 & 0.0021 \\
\hline 8 & 3 & 5 & 6.5 & 8 & 2 & 6 & 6.5 & 7149.7336 & 0.0016 \\
\hline 6 & 1 & 5 & 4.5 & 5 & 2 & 4 & 3.5 & 7162.8575 & -0.0006 \\
\hline 6 & 1 & 5 & 7.5 & 5 & 2 & 4 & 6.5 & 7165.6074 & 0.0031 \\
\hline 6 & 1 & 5 & 5.5 & 5 & 2 & 4 & 4.5 & 7175.0629 & 0.0004 \\
\hline 6 & 1 & 5 & 6.5 & 5 & 2 & 4 & 5.5 & 7177.6583 & -0.0007 \\
\hline 5 & 0 & 5 & 5.5 & 4 & 1 & 4 & 5.5 & 7211.0484 & -0.0017 \\
\hline 5 & 0 & 5 & 3.5 & 4 & 1 & 4 & 2.5 & 7213.1347 & -0.0010 \\
\hline 5 & 0 & 5 & 6.5 & 4 & 1 & 4 & 5.5 & 7213.4875 & 0.0001 \\
\hline 5 & 0 & 5 & 4.5 & 4 & 1 & 4 & 3.5 & 7216.4948 & 0.0003 \\
\hline 5 & 0 & 5 & 5.5 & 4 & 1 & 4 & 4.5 & 7218.7666 & -0.0001 \\
\hline 5 & 0 & 5 & 4.5 & 4 & 1 & 4 & 4.5 & 7219.3583 & -0.0033 \\
\hline 5 & 0 & 5 & 3.5 & 4 & 1 & 4 & 3.5 & 7219.3978 & 0.0006 \\
\hline 7 & 1 & 6 & 5.5 & 7 & 0 & 7 & 5.5 & 7271.8729 & -0.0041 \\
\hline 7 & 1 & 6 & 8.5 & 7 & 0 & 7 & 8.5 & 7276.7714 & -0.0027 \\
\hline 4 & 1 & 3 & 2.5 & 3 & 1 & 2 & 2.5 & 7288.6465 & 0.0032 \\
\hline 7 & 1 & 6 & 6.5 & 7 & 0 & 7 & 6.5 & 7293.6845 & -0.0014 \\
\hline 7 & 1 & 6 & 7.5 & 7 & 0 & 7 & 7.5 & 7297.5896 & -0.0006 \\
\hline 4 & 1 & 3 & 3.5 & 3 & 1 & 2 & 3.5 & 7298.8205 & -0.0005 \\
\hline 4 & 1 & 3 & 4.5 & 3 & 1 & 2 & 3.5 & 7305.6632 & -0.0001 \\
\hline 4 & 1 & 3 & 5.5 & 3 & 1 & 2 & 4.5 & 7305.9335 & -0.0001 \\
\hline 4 & 1 & 3 & 3.5 & 3 & 1 & 2 & 2.5 & 7308.0502 & 0.0003 \\
\hline 4 & 1 & 3 & 2.5 & 3 & 1 & 2 & 1.5 & 7308.2787 & 0.0015 \\
\hline 4 & 1 & 3 & 4.5 & 3 & 1 & 2 & 4.5 & 7325.3961 & -0.0010 \\
\hline 4 & 1 & 4 & 4.5 & 3 & 0 & 3 & 3.5 & 7386.5995 & 0.0004 \\
\hline 4 & 1 & 4 & 3.5 & 3 & 0 & 3 & 3.5 & 7389.4654 & -0.0007 \\
\hline 4 & 1 & 4 & 4.5 & 3 & 0 & 3 & 4.5 & 7390.6631 & -0.0004 \\
\hline 4 & 1 & 4 & 3.5 & 3 & 0 & 3 & 2.5 & 7391.3351 & -0.0003 \\
\hline 4 & 1 & 4 & 2.5 & 3 & 0 & 3 & 2.5 & 7397.5955 & -0.0015 \\
\hline 4 & 1 & 4 & 5.5 & 3 & 0 & 3 & 4.5 & 7398.3804 & 0.0002 \\
\hline 4 & 1 & 4 & 2.5 & 3 & 0 & 3 & 1.5 & 7401.7112 & -0.0008 \\
\hline 5 & 2 & 4 & 3.5 & 5 & 1 & 5 & 3.5 & 7532.5510 & 0.0001 \\
\hline 5 & 2 & 4 & 6.5 & 5 & 1 & 5 & 6.5 & 7535.9167 & 0.0007 \\
\hline 5 & 2 & 4 & 4.5 & 5 & 1 & 5 & 4.5 & 7544.4904 & -0.0003 \\
\hline 5 & 2 & 4 & 5.5 & 5 & 1 & 5 & 5.5 & 7547.9524 & 0.0002 \\
\hline 7 & 3 & 4 & 7.5 & 7 & 2 & 5 & 7.5 & 7557.1390 & 0.0009 \\
\hline
\end{tabular}


Table S2: Observed Transition Frequencies (in $\mathrm{MHz}$ ) for $\mathrm{CF}_{2} \mathrm{CH}^{35} \mathrm{Cl}-\mathrm{HCCH}$

\begin{tabular}{|c|c|c|c|c|c|c|c|c|c|}
\hline$J^{\prime}$ & $K_{a}^{\prime}$ & $K_{c}{ }^{\prime}$ & $F^{\prime}$ & $J^{\prime \prime}$ & $K_{a}^{\prime \prime}$ & $K_{c}^{\prime \prime}$ & $F^{\prime \prime}$ & Observed & Obs - Calc \\
\hline 7 & 3 & 4 & 6.5 & 7 & 2 & 5 & 6.5 & 7559.0059 & -0.0006 \\
\hline 7 & 3 & 4 & 8.5 & 7 & 2 & 5 & 8.5 & 7566.0862 & 0.0024 \\
\hline 7 & 3 & 4 & 5.5 & 7 & 2 & 5 & 5.5 & 7567.8206 & -0.0015 \\
\hline 5 & 1 & 5 & 5.5 & 4 & 1 & 4 & 5.5 & 7764.4308 & -0.0020 \\
\hline 5 & 1 & 5 & 4.5 & 4 & 1 & 4 & 3.5 & 7771.4808 & 0.0003 \\
\hline 5 & 1 & 5 & 5.5 & 4 & 1 & 4 & 4.5 & 7772.1496 & 0.0001 \\
\hline 5 & 1 & 5 & 6.5 & 4 & 1 & 4 & 5.5 & 7772.2047 & 0.0003 \\
\hline 5 & 1 & 5 & 3.5 & 4 & 1 & 4 & 2.5 & 7773.0500 & -0.0002 \\
\hline 5 & 1 & 5 & 4.5 & 4 & 1 & 4 & 4.5 & 7774.3472 & -0.0004 \\
\hline 5 & 1 & 5 & 3.5 & 4 & 1 & 4 & 3.5 & 7779.3119 & 0.0002 \\
\hline 6 & 3 & 3 & 6.5 & 6 & 2 & 4 & 6.5 & 8025.7842 & 0.0016 \\
\hline 6 & 3 & 3 & 5.5 & 6 & 2 & 4 & 5.5 & 8028.3344 & 0.0002 \\
\hline 6 & 3 & 3 & 7.5 & 6 & 2 & 4 & 7.5 & 8036.5424 & 0.0000 \\
\hline 6 & 3 & 3 & 4.5 & 6 & 2 & 4 & 4.5 & 8039.0795 & -0.0002 \\
\hline 5 & 0 & 5 & 5.5 & 4 & 0 & 4 & 4.5 & 8043.3467 & -0.0002 \\
\hline 5 & 0 & 5 & 4.5 & 4 & 0 & 4 & 4.5 & 8043.9388 & -0.0029 \\
\hline 5 & 0 & 5 & 5.5 & 4 & 0 & 4 & 5.5 & 8043.9537 & 0.0019 \\
\hline 5 & 0 & 5 & 4.5 & 4 & 0 & 4 & 3.5 & 8044.1009 & -0.0006 \\
\hline 5 & 0 & 5 & 6.5 & 4 & 0 & 4 & 5.5 & 8046.3891 & 0.0000 \\
\hline 5 & 0 & 5 & 3.5 & 4 & 0 & 4 & 3.5 & 8047.0029 & -0.0012 \\
\hline 5 & 0 & 5 & 3.5 & 4 & 0 & 4 & 2.5 & 8047.7885 & 0.0003 \\
\hline 6 & 2 & 5 & 4.5 & 6 & 1 & 6 & 4.5 & 8386.0927 & -0.0003 \\
\hline 6 & 2 & 5 & 7.5 & 6 & 1 & 6 & 7.5 & 8389.0787 & 0.0004 \\
\hline 6 & 2 & 5 & 5.5 & 6 & 1 & 6 & 5.5 & 8398.8135 & -0.0022 \\
\hline 6 & 2 & 5 & 6.5 & 6 & 1 & 6 & 6.5 & 8401.9078 & -0.0014 \\
\hline 5 & 3 & 2 & 5.5 & 5 & 2 & 3 & 5.5 & 8449.5087 & 0.0006 \\
\hline 5 & 3 & 2 & 4.5 & 5 & 2 & 3 & 4.5 & 8452.8790 & -0.0013 \\
\hline 5 & 3 & 2 & 6.5 & 5 & 2 & 3 & 6.5 & 8461.3839 & 0.0004 \\
\hline 5 & 3 & 2 & 3.5 & 5 & 2 & 3 & 3.5 & 8464.6686 & -0.0017 \\
\hline 5 & 2 & 4 & 3.5 & 4 & 2 & 3 & 3.5 & 8478.9763 & 0.0004 \\
\hline 5 & 2 & 4 & 4.5 & 4 & 2 & 3 & 4.5 & 8481.7026 & 0.0009 \\
\hline 5 & 2 & 4 & 3.5 & 4 & 2 & 3 & 2.5 & 8481.7868 & 0.0004 \\
\hline 5 & 2 & 4 & 6.5 & 4 & 2 & 3 & 5.5 & 8482.0363 & 0.0001 \\
\hline 5 & 2 & 4 & 5.5 & 4 & 2 & 3 & 4.5 & 8482.9651 & 0.0000 \\
\hline 5 & 2 & 4 & 4.5 & 4 & 2 & 3 & 3.5 & 8483.0850 & 0.0005 \\
\hline 5 & 2 & 4 & 5.5 & 4 & 2 & 3 & 5.5 & 8486.2986 & -0.0022 \\
\hline 5 & 1 & 5 & 5.5 & 4 & 0 & 4 & 4.5 & 8596.7294 & -0.0002 \\
\hline 5 & 1 & 5 & 5.5 & 4 & 0 & 4 & 5.5 & 8597.3336 & -0.0009 \\
\hline 5 & 1 & 5 & 4.5 & 4 & 0 & 4 & 4.5 & 8598.9260 & -0.0017 \\
\hline 5 & 1 & 5 & 4.5 & 4 & 0 & 4 & 3.5 & 8599.0876 & 0.0002 \\
\hline 5 & 1 & 5 & 6.5 & 4 & 0 & 4 & 5.5 & 8605.1057 & -0.0003 \\
\hline 5 & 4 & 2 & 3.5 & 4 & 4 & 1 & 2.5 & 8606.3017 & -0.0013 \\
\hline 5 & 1 & 5 & 3.5 & 4 & 0 & 4 & 3.5 & 8606.9170 & -0.0017 \\
\hline
\end{tabular}


Table S2: Observed Transition Frequencies (in $\mathrm{MHz}$ ) for $\mathrm{CF}_{2} \mathrm{CH}^{35} \mathrm{Cl}-\mathrm{HCCH}$

\begin{tabular}{|c|c|c|c|c|c|c|c|c|c|}
\hline$J^{\prime}$ & $K_{a}^{\prime}$ & $K_{c}^{\prime}$ & $F^{\prime}$ & $J^{\prime \prime}$ & $K_{a}^{\prime \prime}$ & $K_{c}^{\prime \prime}$ & $F^{\prime \prime}$ & Observed & Obs - Calc \\
\hline 5 & 4 & 1 & 3.5 & 4 & 4 & 0 & 2.5 & 8607.2062 & -0.0003 \\
\hline 5 & 1 & 5 & 3.5 & 4 & 0 & 4 & 2.5 & 8607.7029 & 0.0001 \\
\hline 5 & 4 & 2 & 6.5 & 4 & 4 & 1 & 5.5 & 8609.4071 & -0.0017 \\
\hline 5 & 4 & 1 & 6.5 & 4 & 4 & 0 & 5.5 & 8610.3001 & -0.0008 \\
\hline 5 & 4 & 2 & 5.5 & 4 & 4 & 1 & 4.5 & 8617.4042 & 0.0013 \\
\hline 5 & 4 & 1 & 5.5 & 4 & 4 & 0 & 4.5 & 8618.3246 & -0.0001 \\
\hline 5 & 3 & 3 & 3.5 & 4 & 3 & 2 & 2.5 & 8625.2172 & 0.0006 \\
\hline 5 & 3 & 3 & 6.5 & 4 & 3 & 2 & 5.5 & 8626.6373 & -0.0001 \\
\hline 5 & 3 & 3 & 3.5 & 4 & 3 & 2 & 3.5 & 8626.9719 & 0.0005 \\
\hline 5 & 3 & 3 & 5.5 & 4 & 3 & 2 & 5.5 & 8629.5070 & -0.0020 \\
\hline 5 & 3 & 3 & 4.5 & 4 & 3 & 2 & 3.5 & 8629.8215 & 0.0007 \\
\hline 5 & 3 & 3 & 4.5 & 4 & 3 & 2 & 4.5 & 8630.4470 & -0.0012 \\
\hline 5 & 3 & 3 & 5.5 & 4 & 3 & 2 & 4.5 & 8631.2558 & 0.0005 \\
\hline 5 & 3 & 2 & 3.5 & 4 & 3 & 1 & 2.5 & 8666.1275 & 0.0005 \\
\hline 5 & 3 & 2 & 3.5 & 4 & 3 & 1 & 3.5 & 8667.5139 & -0.0009 \\
\hline 5 & 3 & 2 & 6.5 & 4 & 3 & 1 & 5.5 & 8667.7624 & -0.0003 \\
\hline 5 & 3 & 2 & 4.5 & 4 & 3 & 1 & 3.5 & 8671.5214 & 0.0001 \\
\hline 5 & 3 & 2 & 5.5 & 4 & 3 & 1 & 5.5 & 8671.8072 & -0.0030 \\
\hline 5 & 3 & 2 & 4.5 & 4 & 3 & 1 & 4.5 & 8671.9152 & -0.0016 \\
\hline 5 & 3 & 2 & 5.5 & 4 & 3 & 1 & 4.5 & 8673.0645 & -0.0001 \\
\hline 2 & 2 & 1 & 2.5 & 1 & 1 & 0 & 1.5 & 8720.9569 & -0.0003 \\
\hline 2 & 2 & 1 & 1.5 & 1 & 1 & 0 & 1.5 & 8727.4549 & -0.0010 \\
\hline 2 & 2 & 1 & 2.5 & 1 & 1 & 0 & 2.5 & 8730.0069 & -0.0015 \\
\hline 2 & 2 & 1 & 1.5 & 1 & 1 & 0 & 2.5 & 8736.5089 & 0.0018 \\
\hline 2 & 2 & 1 & 3.5 & 1 & 1 & 0 & 2.5 & 8739.0543 & 0.0008 \\
\hline 2 & 2 & 1 & 1.5 & 1 & 1 & 0 & 0.5 & 8743.8154 & 0.0006 \\
\hline 2 & 2 & 1 & 0.5 & 1 & 1 & 0 & 0.5 & 8752.8564 & 0.0007 \\
\hline 4 & 3 & 1 & 4.5 & 4 & 2 & 2 & 4.5 & 8762.4983 & -0.0006 \\
\hline 4 & 3 & 1 & 3.5 & 4 & 2 & 2 & 4.5 & 8762.8939 & -0.0006 \\
\hline 4 & 3 & 1 & 5.5 & 4 & 2 & 2 & 4.5 & 8763.7549 & 0.0015 \\
\hline 4 & 3 & 1 & 4.5 & 4 & 2 & 2 & 3.5 & 8766.5544 & -0.0006 \\
\hline 4 & 3 & 1 & 3.5 & 4 & 2 & 2 & 3.5 & 8766.9491 & -0.0013 \\
\hline 4 & 3 & 1 & 2.5 & 4 & 2 & 2 & 3.5 & 8768.3391 & 0.0010 \\
\hline 4 & 3 & 1 & 5.5 & 4 & 2 & 2 & 5.5 & 8775.2928 & 0.0013 \\
\hline 4 & 3 & 1 & 3.5 & 4 & 2 & 2 & 2.5 & 8778.4348 & -0.0020 \\
\hline 4 & 3 & 1 & 2.5 & 4 & 2 & 2 & 2.5 & 8779.8249 & 0.0004 \\
\hline 8 & 1 & 7 & 9.5 & 8 & 0 & 8 & 9.5 & 8903.9965 & 0.0002 \\
\hline 6 & 0 & 6 & 6.5 & 5 & 1 & 5 & 6.5 & 8916.9861 & -0.0024 \\
\hline 8 & 1 & 7 & 7.5 & 8 & 0 & 8 & 7.5 & 8920.2712 & -0.0008 \\
\hline 6 & 0 & 6 & 4.5 & 5 & 1 & 5 & 3.5 & 8921.4787 & 0.0000 \\
\hline 6 & 0 & 6 & 7.5 & 5 & 1 & 5 & 6.5 & 8922.3496 & 0.0002 \\
\hline 6 & 0 & 6 & 5.5 & 5 & 1 & 5 & 4.5 & 8923.8058 & -0.0005 \\
\hline 6 & 0 & 6 & 6.5 & 5 & 1 & 5 & 5.5 & 8924.7599 & -0.0001 \\
\hline
\end{tabular}


Table S2: Observed Transition Frequencies (in $\mathrm{MHz}$ ) for $\mathrm{CF}_{2} \mathrm{CH}^{35} \mathrm{Cl}-\mathrm{HCCH}$

\begin{tabular}{|c|c|c|c|c|c|c|c|c|c|}
\hline$J^{\prime}$ & $K_{a}^{\prime}$ & $K_{c}{ }^{\prime}$ & $F^{\prime}$ & $J^{\prime \prime}$ & $K_{a}^{\prime \prime}$ & $K_{c}^{\prime \prime}$ & $F^{\prime \prime}$ & Observed & Obs - Calc \\
\hline 6 & 0 & 6 & 5.5 & 5 & 1 & 5 & 5.5 & 8926.0029 & -0.0015 \\
\hline 6 & 0 & 6 & 4.5 & 5 & 1 & 5 & 4.5 & 8929.3116 & 0.0017 \\
\hline 3 & 3 & 0 & 3.5 & 3 & 2 & 1 & 3.5 & 8945.9358 & -0.0027 \\
\hline 3 & 3 & 0 & 3.5 & 3 & 2 & 1 & 2.5 & 8948.2890 & -0.0005 \\
\hline 3 & 3 & 0 & 2.5 & 3 & 2 & 1 & 3.5 & 8950.8918 & -0.0028 \\
\hline 3 & 3 & 0 & 3.5 & 3 & 2 & 1 & 4.5 & 8950.9492 & -0.0023 \\
\hline 3 & 3 & 0 & 2.5 & 3 & 2 & 1 & 2.5 & 8953.2443 & -0.0013 \\
\hline 3 & 3 & 0 & 4.5 & 3 & 2 & 1 & 3.5 & 8956.5436 & 0.0011 \\
\hline 3 & 3 & 0 & 2.5 & 3 & 2 & 1 & 1.5 & 8958.1697 & -0.0003 \\
\hline 3 & 3 & 0 & 4.5 & 3 & 2 & 1 & 4.5 & 8961.5564 & 0.0009 \\
\hline 3 & 3 & 0 & 1.5 & 3 & 2 & 1 & 2.5 & 8963.8788 & 0.0023 \\
\hline 3 & 3 & 0 & 1.5 & 3 & 2 & 1 & 1.5 & 8968.8031 & 0.0023 \\
\hline 5 & 2 & 3 & 3.5 & 4 & 2 & 2 & 3.5 & 8969.7945 & -0.0004 \\
\hline 5 & 2 & 3 & 3.5 & 4 & 2 & 2 & 2.5 & 8981.2817 & 0.0004 \\
\hline 5 & 2 & 3 & 4.5 & 4 & 2 & 2 & 4.5 & 8981.5347 & -0.0008 \\
\hline 5 & 2 & 3 & 6.5 & 4 & 2 & 2 & 5.5 & 8981.6707 & 0.0000 \\
\hline 5 & 2 & 3 & 4.5 & 4 & 2 & 2 & 3.5 & 8985.5916 & 0.0002 \\
\hline 5 & 2 & 3 & 5.5 & 4 & 2 & 2 & 4.5 & 8986.0550 & -0.0004 \\
\hline 5 & 2 & 3 & 5.5 & 4 & 2 & 2 & 5.5 & 8997.5917 & -0.0019 \\
\hline 2 & 2 & 0 & 1.5 & 1 & 1 & 1 & 0.5 & 9012.4185 & 0.0008 \\
\hline 2 & 2 & 0 & 0.5 & 1 & 1 & 1 & 0.5 & 9019.4982 & 0.0009 \\
\hline 2 & 2 & 0 & 2.5 & 1 & 1 & 1 & 2.5 & 9022.0015 & -0.0012 \\
\hline 2 & 2 & 0 & 1.5 & 1 & 1 & 1 & 2.5 & 9026.9665 & -0.0025 \\
\hline 2 & 2 & 0 & 3.5 & 1 & 1 & 1 & 2.5 & 9028.9689 & 0.0010 \\
\hline 2 & 2 & 0 & 2.5 & 1 & 1 & 1 & 1.5 & 9040.1122 & -0.0004 \\
\hline 2 & 2 & 0 & 1.5 & 1 & 1 & 1 & 1.5 & 9045.0784 & -0.0005 \\
\hline 5 & 1 & 4 & 3.5 & 4 & 1 & 3 & 3.5 & 9046.3112 & 0.0005 \\
\hline 2 & 2 & 0 & 0.5 & 1 & 1 & 1 & 1.5 & 9052.1604 & 0.0019 \\
\hline 5 & 1 & 4 & 4.5 & 4 & 1 & 3 & 4.5 & 9057.6912 & -0.0011 \\
\hline 5 & 1 & 4 & 5.5 & 4 & 1 & 3 & 4.5 & 9062.8552 & -0.0001 \\
\hline 5 & 1 & 4 & 6.5 & 4 & 1 & 3 & 5.5 & 9064.0570 & 0.0002 \\
\hline 5 & 1 & 4 & 4.5 & 4 & 1 & 3 & 3.5 & 9064.5345 & -0.0001 \\
\hline 5 & 1 & 4 & 3.5 & 4 & 1 & 3 & 2.5 & 9065.7175 & 0.0002 \\
\hline 5 & 1 & 4 & 5.5 & 4 & 1 & 3 & 5.5 & 9082.3170 & -0.0019 \\
\hline 3 & 3 & 1 & 3.5 & 3 & 2 & 2 & 2.5 & 9089.8808 & -0.0008 \\
\hline 3 & 3 & 1 & 3.5 & 3 & 2 & 2 & 4.5 & 9090.7378 & -0.0035 \\
\hline 3 & 3 & 1 & 3.5 & 3 & 2 & 2 & 3.5 & 9091.0559 & -0.0026 \\
\hline 3 & 3 & 1 & 2.5 & 3 & 2 & 2 & 2.5 & 9094.8865 & -0.0017 \\
\hline 3 & 3 & 1 & 2.5 & 3 & 2 & 2 & 3.5 & 9096.0633 & -0.0018 \\
\hline 3 & 3 & 1 & 2.5 & 3 & 2 & 2 & 1.5 & 9096.1860 & 0.0006 \\
\hline 3 & 3 & 1 & 4.5 & 3 & 2 & 2 & 4.5 & 9101.4563 & 0.0016 \\
\hline 3 & 3 & 1 & 4.5 & 3 & 2 & 2 & 3.5 & 9101.7761 & 0.0043 \\
\hline 3 & 3 & 1 & 1.5 & 3 & 2 & 2 & 2.5 & 9105.6251 & -0.0008 \\
\hline
\end{tabular}


Table S2: Observed Transition Frequencies (in $\mathrm{MHz}$ ) for $\mathrm{CF}_{2} \mathrm{CH}^{35} \mathrm{Cl}-\mathrm{HCCH}$

\begin{tabular}{|c|c|c|c|c|c|c|c|c|c|}
\hline$J^{\prime}$ & $K_{a}^{\prime}$ & $K_{c}^{\prime}$ & $F^{\prime}$ & $J^{\prime \prime}$ & $K_{a}^{\prime \prime}$ & $K_{c}^{\prime \prime}$ & $F^{\prime \prime}$ & Observed & Obs - Calc \\
\hline 3 & 3 & 1 & 1.5 & 3 & 2 & 2 & 1.5 & 9106.9254 & 0.0024 \\
\hline 4 & 3 & 2 & 4.5 & 4 & 2 & 3 & 4.5 & 9171.0297 & -0.0009 \\
\hline 4 & 3 & 2 & 3.5 & 4 & 2 & 3 & 4.5 & 9171.6555 & -0.0026 \\
\hline 4 & 3 & 2 & 4.5 & 4 & 2 & 3 & 3.5 & 9172.4144 & 0.0010 \\
\hline 4 & 3 & 2 & 5.5 & 4 & 2 & 3 & 4.5 & 9172.7791 & 0.0022 \\
\hline 4 & 3 & 2 & 3.5 & 4 & 2 & 3 & 3.5 & 9173.0392 & -0.0016 \\
\hline 4 & 3 & 2 & 4.5 & 4 & 2 & 3 & 5.5 & 9174.3641 & -0.0022 \\
\hline 4 & 3 & 2 & 2.5 & 4 & 2 & 3 & 3.5 & 9174.7951 & -0.0006 \\
\hline 4 & 3 & 2 & 3.5 & 4 & 2 & 3 & 2.5 & 9175.8517 & 0.0004 \\
\hline 4 & 3 & 2 & 5.5 & 4 & 2 & 3 & 5.5 & 9176.1133 & 0.0007 \\
\hline 4 & 3 & 2 & 2.5 & 4 & 2 & 3 & 2.5 & 9177.6051 & -0.0010 \\
\hline 6 & 1 & 6 & 5.5 & 5 & 1 & 5 & 4.5 & 9271.9131 & -0.0009 \\
\hline 6 & 1 & 6 & 6.5 & 5 & 1 & 5 & 5.5 & 9272.1003 & 0.0001 \\
\hline 6 & 1 & 6 & 4.5 & 5 & 1 & 5 & 3.5 & 9272.6005 & -0.0006 \\
\hline 6 & 1 & 6 & 5.5 & 5 & 1 & 5 & 5.5 & 9274.1112 & -0.0008 \\
\hline 6 & 1 & 6 & 4.5 & 5 & 1 & 5 & 4.5 & 9280.4323 & 0.0000 \\
\hline 5 & 3 & 3 & 3.5 & 5 & 2 & 4 & 4.5 & 9316.9295 & 0.0017 \\
\hline 5 & 3 & 3 & 4.5 & 5 & 2 & 4 & 5.5 & 9318.5135 & -0.0002 \\
\hline 5 & 3 & 3 & 5.5 & 5 & 2 & 4 & 5.5 & 9319.3206 & -0.0002 \\
\hline 5 & 3 & 3 & 4.5 & 5 & 2 & 4 & 4.5 & 9319.7758 & -0.0013 \\
\hline 5 & 3 & 3 & 5.5 & 5 & 2 & 4 & 4.5 & 9320.5865 & 0.0023 \\
\hline 5 & 3 & 3 & 6.5 & 5 & 2 & 4 & 6.5 & 9320.7141 & 0.0003 \\
\hline 5 & 3 & 3 & 3.5 & 5 & 2 & 4 & 3.5 & 9321.0367 & 0.0004 \\
\hline 5 & 3 & 3 & 5.5 & 5 & 2 & 4 & 6.5 & 9323.5839 & -0.0015 \\
\hline 5 & 3 & 3 & 4.5 & 5 & 2 & 4 & 3.5 & 9323.8864 & 0.0007 \\
\hline 7 & 2 & 6 & 5.5 & 7 & 1 & 7 & 5.5 & 9373.3653 & -0.0002 \\
\hline 7 & 2 & 6 & 8.5 & 7 & 1 & 7 & 8.5 & 9376.0257 & 0.0009 \\
\hline 7 & 2 & 6 & 6.5 & 7 & 1 & 7 & 6.5 & 9386.4190 & -0.0001 \\
\hline 7 & 2 & 6 & 7.5 & 7 & 1 & 7 & 7.5 & 9389.1547 & 0.0023 \\
\hline 7 & 1 & 6 & 5.5 & 6 & 2 & 5 & 4.5 & 9432.7021 & 0.0011 \\
\hline 7 & 1 & 6 & 8.5 & 6 & 2 & 5 & 7.5 & 9435.0589 & 0.0000 \\
\hline 7 & 1 & 6 & 6.5 & 6 & 2 & 5 & 5.5 & 9442.7107 & 0.0003 \\
\hline 7 & 1 & 6 & 7.5 & 6 & 2 & 5 & 6.5 & 9444.0185 & -0.0010 \\
\hline 6 & 0 & 6 & 6.5 & 5 & 0 & 5 & 6.5 & 9475.7043 & -0.0011 \\
\hline 6 & 0 & 6 & 6.5 & 5 & 0 & 5 & 5.5 & 9478.1426 & -0.0001 \\
\hline 6 & 0 & 6 & 5.5 & 5 & 0 & 5 & 4.5 & 9478.7919 & -0.0004 \\
\hline 6 & 0 & 6 & 5.5 & 5 & 0 & 5 & 5.5 & 9479.3862 & -0.0010 \\
\hline 6 & 0 & 6 & 7.5 & 5 & 0 & 5 & 6.5 & 9481.0663 & 0.0000 \\
\hline 6 & 0 & 6 & 4.5 & 5 & 0 & 5 & 3.5 & 9481.3930 & -0.0002 \\
\hline 6 & 0 & 6 & 4.5 & 5 & 0 & 5 & 4.5 & 9484.2950 & -0.0009 \\
\hline 6 & 3 & 4 & 4.5 & 6 & 2 & 5 & 4.5 & 9560.4106 & -0.0014 \\
\hline 6 & 3 & 4 & 7.5 & 6 & 2 & 5 & 7.5 & 9561.2206 & -0.0005 \\
\hline 6 & 3 & 4 & 6.5 & 6 & 2 & 5 & 6.5 & 9561.9655 & -0.0012 \\
\hline
\end{tabular}


Table S2: Observed Transition Frequencies (in $\mathrm{MHz}$ ) for $\mathrm{CF}_{2} \mathrm{CH}^{35} \mathrm{Cl}-\mathrm{HCCH}$

\begin{tabular}{|c|c|c|c|c|c|c|c|c|c|}
\hline$J^{\prime}$ & $K_{a}^{\prime}$ & $K_{c}^{\prime}$ & $F^{\prime}$ & $J^{\prime \prime}$ & $K_{a}^{\prime \prime}$ & $K_{c}^{\prime \prime}$ & $F^{\prime \prime}$ & Observed & Obs - Calc \\
\hline 6 & 3 & 4 & 5.5 & 6 & 2 & 5 & 5.5 & 9562.2187 & -0.0008 \\
\hline 6 & 1 & 6 & 6.5 & 5 & 0 & 5 & 6.5 & 9823.0435 & -0.0021 \\
\hline 6 & 1 & 6 & 6.5 & 5 & 0 & 5 & 5.5 & 9825.4826 & -0.0003 \\
\hline 6 & 1 & 6 & 5.5 & 5 & 0 & 5 & 4.5 & 9826.8995 & -0.0004 \\
\hline 6 & 1 & 6 & 5.5 & 5 & 0 & 5 & 5.5 & 9827.4937 & -0.0011 \\
\hline 6 & 1 & 6 & 7.5 & 5 & 0 & 5 & 6.5 & 9831.5208 & -0.0001 \\
\hline 6 & 1 & 6 & 4.5 & 5 & 0 & 5 & 3.5 & 9832.5153 & -0.0003 \\
\hline 6 & 1 & 6 & 4.5 & 5 & 0 & 5 & 4.5 & 9835.4177 & -0.0006 \\
\hline 7 & 3 & 5 & 5.5 & 7 & 2 & 6 & 5.5 & 9917.3524 & -0.0005 \\
\hline 7 & 3 & 5 & 8.5 & 7 & 2 & 6 & 8.5 & 9918.0247 & 0.0006 \\
\hline 7 & 3 & 5 & 6.5 & 7 & 2 & 6 & 6.5 & 9920.6158 & 0.0002 \\
\hline 7 & 3 & 5 & 7.5 & 7 & 2 & 6 & 7.5 & 9921.2495 & 0.0001 \\
\hline 6 & 2 & 5 & 4.5 & 5 & 2 & 4 & 4.5 & 10122.0346 & 0.0000 \\
\hline 6 & 2 & 5 & 5.5 & 5 & 2 & 4 & 5.5 & 10124.9740 & -0.0016 \\
\hline 6 & 2 & 5 & 7.5 & 5 & 2 & 4 & 6.5 & 10125.9668 & 0.0005 \\
\hline 6 & 2 & 5 & 6.5 & 5 & 2 & 4 & 5.5 & 10126.0567 & -0.0004 \\
\hline 6 & 2 & 5 & 4.5 & 5 & 2 & 4 & 3.5 & 10126.1430 & -0.0002 \\
\hline 6 & 2 & 5 & 5.5 & 5 & 2 & 4 & 4.5 & 10126.2385 & -0.0005 \\
\hline 6 & 2 & 5 & 6.5 & 5 & 2 & 4 & 6.5 & 10130.3206 & -0.0011 \\
\hline 3 & 2 & 2 & 3.5 & 2 & 1 & 1 & 2.5 & 10164.5416 & -0.0010 \\
\hline 3 & 2 & 2 & 2.5 & 2 & 1 & 1 & 2.5 & 10165.7179 & -0.0015 \\
\hline 3 & 2 & 2 & 1.5 & 2 & 1 & 1 & 1.5 & 10177.4181 & 0.0004 \\
\hline 3 & 2 & 2 & 2.5 & 2 & 1 & 1 & 1.5 & 10178.7137 & -0.0012 \\
\hline 3 & 2 & 2 & 3.5 & 2 & 1 & 1 & 3.5 & 10182.6966 & -0.0008 \\
\hline 3 & 2 & 2 & 4.5 & 2 & 1 & 1 & 3.5 & 10183.0150 & 0.0004 \\
\hline 3 & 2 & 2 & 1.5 & 2 & 1 & 1 & 0.5 & 10195.4928 & 0.0005 \\
\hline 6 & 5 & 2 & 4.5 & 5 & 5 & 1 & 3.5 & 10325.0849 & 0.0008 \\
\hline 6 & 5 & 1 & 4.5 & 5 & 5 & 0 & 3.5 & 10325.1400 & -0.0027 \\
\hline 6 & 5 & 2 & 7.5 & 5 & 5 & 1 & 6.5 & 10327.3598 & -0.0015 \\
\hline 6 & 5 & 1 & 7.5 & 5 & 5 & 0 & 6.5 & 10327.4169 & -0.0035 \\
\hline 6 & 5 & 2 & 5.5 & 5 & 5 & 1 & 4.5 & 10332.1801 & 0.0022 \\
\hline 6 & 5 & 1 & 5.5 & 5 & 5 & 0 & 4.5 & 10332.2370 & -0.0009 \\
\hline 6 & 5 & 2 & 6.5 & 5 & 5 & 1 & 5.5 & 10334.4653 & 0.0010 \\
\hline 6 & 5 & 1 & 6.5 & 5 & 5 & 0 & 5.5 & 10334.5237 & -0.0010 \\
\hline 6 & 4 & 3 & 4.5 & 5 & 4 & 2 & 3.5 & 10351.5296 & -0.0005 \\
\hline 6 & 4 & 3 & 7.5 & 5 & 4 & 2 & 6.5 & 10352.8478 & -0.0006 \\
\hline 6 & 4 & 3 & 6.5 & 5 & 4 & 2 & 6.5 & 10353.4000 & -0.0039 \\
\hline 6 & 4 & 2 & 4.5 & 5 & 4 & 1 & 3.5 & 10355.5823 & -0.0004 \\
\hline 6 & 4 & 3 & 4.5 & 5 & 4 & 2 & 4.5 & 10355.7483 & 0.0016 \\
\hline 6 & 4 & 3 & 5.5 & 5 & 4 & 2 & 4.5 & 10356.3137 & 0.0008 \\
\hline 6 & 4 & 2 & 7.5 & 5 & 4 & 1 & 6.5 & 10356.9771 & -0.0010 \\
\hline 6 & 4 & 3 & 6.5 & 5 & 4 & 2 & 5.5 & 10357.5976 & 0.0003 \\
\hline 6 & 4 & 2 & 4.5 & 5 & 4 & 1 & 4.5 & 10359.7703 & 0.0006 \\
\hline
\end{tabular}


Table S2: Observed Transition Frequencies (in $\mathrm{MHz}$ ) for $\mathrm{CF}_{2} \mathrm{CH}^{35} \mathrm{Cl}-\mathrm{HCCH}$

\begin{tabular}{|c|c|c|c|c|c|c|c|c|c|}
\hline$J^{\prime}$ & $K_{a}^{\prime}$ & $K_{c}^{\prime}$ & $F^{\prime}$ & $J^{\prime \prime}$ & $K_{a}^{\prime \prime}$ & $K_{c}^{\prime \prime}$ & $F^{\prime \prime}$ & Observed & Obs - Calc \\
\hline 6 & 4 & 2 & 5.5 & 5 & 4 & 1 & 4.5 & 10360.4901 & 0.0003 \\
\hline 6 & 4 & 2 & 6.5 & 5 & 4 & 1 & 5.5 & 10361.7573 & -0.0003 \\
\hline 6 & 3 & 4 & 4.5 & 5 & 3 & 3 & 4.5 & 10362.6702 & 0.0008 \\
\hline 6 & 3 & 4 & 4.5 & 5 & 3 & 3 & 3.5 & 10365.5193 & 0.0005 \\
\hline 6 & 3 & 4 & 7.5 & 5 & 3 & 3 & 6.5 & 10366.4740 & 0.0004 \\
\hline 6 & 3 & 4 & 5.5 & 5 & 3 & 3 & 5.5 & 10367.8730 & -0.0013 \\
\hline 6 & 3 & 4 & 5.5 & 5 & 3 & 3 & 4.5 & 10368.6823 & 0.0010 \\
\hline 6 & 3 & 4 & 6.5 & 5 & 3 & 3 & 5.5 & 10368.7029 & -0.0001 \\
\hline 6 & 3 & 4 & 6.5 & 5 & 3 & 3 & 6.5 & 10371.5720 & -0.0026 \\
\hline 8 & 3 & 6 & 6.5 & 8 & 2 & 7 & 6.5 & 10408.5576 & 0.0007 \\
\hline 8 & 3 & 6 & 9.5 & 8 & 2 & 7 & 9.5 & 10409.4305 & 0.0007 \\
\hline 8 & 3 & 6 & 7.5 & 8 & 2 & 7 & 7.5 & 10413.4294 & 0.0012 \\
\hline 8 & 3 & 6 & 8.5 & 8 & 2 & 7 & 8.5 & 10414.3056 & 0.0015 \\
\hline 6 & 3 & 3 & 4.5 & 5 & 3 & 2 & 4.5 & 10467.5648 & -0.0025 \\
\hline 6 & 3 & 3 & 4.5 & 5 & 3 & 2 & 3.5 & 10471.5740 & 0.0002 \\
\hline 6 & 3 & 3 & 7.5 & 5 & 3 & 2 & 6.5 & 10472.3199 & 0.0001 \\
\hline 6 & 3 & 3 & 5.5 & 5 & 3 & 2 & 5.5 & 10474.3210 & -0.0016 \\
\hline 6 & 3 & 3 & 5.5 & 5 & 3 & 2 & 4.5 & 10475.4707 & 0.0002 \\
\hline 6 & 3 & 3 & 6.5 & 5 & 3 & 2 & 5.5 & 10476.1982 & 0.0005 \\
\hline 8 & 2 & 7 & 6.5 & 8 & 1 & 8 & 6.5 & 10479.3056 & 0.0018 \\
\hline 6 & 3 & 3 & 6.5 & 5 & 3 & 2 & 6.5 & 10480.2445 & -0.0007 \\
\hline 8 & 2 & 7 & 9.5 & 8 & 1 & 8 & 9.5 & 10481.6312 & 0.0025 \\
\hline 8 & 2 & 7 & 7.5 & 8 & 1 & 8 & 7.5 & 10492.3442 & 0.0005 \\
\hline 8 & 2 & 7 & 8.5 & 8 & 1 & 8 & 8.5 & 10494.7202 & 0.0007 \\
\hline 7 & 0 & 7 & 7.5 & 6 & 1 & 6 & 7.5 & 10539.8622 & -0.0009 \\
\hline 7 & 0 & 7 & 5.5 & 6 & 1 & 6 & 4.5 & 10546.9169 & 0.0000 \\
\hline 7 & 0 & 7 & 8.5 & 6 & 1 & 6 & 7.5 & 10547.3630 & -0.0001 \\
\hline 7 & 0 & 7 & 6.5 & 6 & 1 & 6 & 5.5 & 10547.8399 & -0.0004 \\
\hline 7 & 0 & 7 & 7.5 & 6 & 1 & 6 & 6.5 & 10548.3385 & 0.0001 \\
\hline 7 & 0 & 7 & 6.5 & 6 & 1 & 6 & 6.5 & 10549.8513 & -0.0008 \\
\hline 7 & 0 & 7 & 5.5 & 6 & 1 & 6 & 5.5 & 10555.4355 & 0.0003 \\
\hline 9 & 1 & 8 & 7.5 & 9 & 0 & 9 & 7.5 & 10574.0659 & -0.0018 \\
\hline 9 & 1 & 8 & 10.5 & 9 & 0 & 9 & 10.5 & 10576.9097 & 0.0000 \\
\hline 9 & 1 & 8 & 8.5 & 9 & 0 & 9 & 8.5 & 10591.8838 & -0.0032 \\
\hline 9 & 1 & 8 & 9.5 & 9 & 0 & 9 & 9.5 & 10594.7622 & 0.0010 \\
\hline 7 & 1 & 7 & 7.5 & 6 & 1 & 6 & 7.5 & 10746.8014 & -0.0026 \\
\hline 6 & 1 & 5 & 4.5 & 5 & 1 & 4 & 4.5 & 10749.1485 & 0.0018 \\
\hline 7 & 1 & 7 & 6.5 & 6 & 1 & 6 & 5.5 & 10755.1548 & -0.0002 \\
\hline 7 & 1 & 7 & 7.5 & 6 & 1 & 6 & 6.5 & 10755.2790 & -0.0002 \\
\hline 7 & 1 & 7 & 5.5 & 6 & 1 & 6 & 4.5 & 10755.9148 & -0.0005 \\
\hline 7 & 1 & 7 & 8.5 & 6 & 1 & 6 & 7.5 & 10756.0451 & 0.0003 \\
\hline 7 & 1 & 7 & 6.5 & 6 & 1 & 6 & 6.5 & 10757.1650 & -0.0018 \\
\hline 6 & 1 & 5 & 5.5 & 5 & 1 & 4 & 5.5 & 10760.2955 & -0.0010 \\
\hline
\end{tabular}


Table S2: Observed Transition Frequencies (in $\mathrm{MHz}$ ) for $\mathrm{CF}_{2} \mathrm{CH}^{35} \mathrm{Cl}-\mathrm{HCCH}$

\begin{tabular}{|c|c|c|c|c|c|c|c|c|c|}
\hline$J^{\prime}$ & $K_{a}^{\prime}$ & $K_{c}{ }^{\prime}$ & $F^{\prime}$ & $J^{\prime \prime}$ & $K_{a}^{\prime \prime}$ & $K_{c}^{\prime \prime}$ & $F^{\prime \prime}$ & Observed & Obs - Calc \\
\hline 6 & 1 & 5 & 6.5 & 5 & 1 & 4 & 5.5 & 10764.1564 & -0.0001 \\
\hline 7 & 1 & 7 & 5.5 & 6 & 1 & 6 & 5.5 & 10764.4316 & -0.0019 \\
\hline 6 & 1 & 5 & 5.5 & 5 & 1 & 4 & 4.5 & 10765.4593 & -0.0004 \\
\hline 6 & 1 & 5 & 7.5 & 5 & 1 & 4 & 6.5 & 10766.0995 & 0.0003 \\
\hline 6 & 1 & 5 & 4.5 & 5 & 1 & 4 & 3.5 & 10767.3705 & 0.0000 \\
\hline 6 & 1 & 5 & 6.5 & 5 & 1 & 4 & 6.5 & 10782.4168 & -0.0018 \\
\hline 6 & 2 & 4 & 4.5 & 5 & 2 & 3 & 4.5 & 10881.3695 & 0.0016 \\
\hline 7 & 0 & 7 & 7.5 & 6 & 0 & 6 & 7.5 & 10890.3167 & -0.0009 \\
\hline 6 & 2 & 4 & 5.5 & 5 & 2 & 3 & 5.5 & 10895.4968 & 0.0003 \\
\hline 7 & 0 & 7 & 7.5 & 6 & 0 & 6 & 6.5 & 10895.6787 & 0.0002 \\
\hline 7 & 0 & 7 & 6.5 & 6 & 0 & 6 & 5.5 & 10895.9482 & 0.0003 \\
\hline 6 & 2 & 4 & 7.5 & 5 & 2 & 3 & 6.5 & \multirow{2}{*}{10897.1621} & \multirow{2}{*}{-0.0005} \\
\hline 6 & 2 & 4 & 4.5 & 5 & 2 & 3 & 3.5 & & \\
\hline 7 & 0 & 7 & 8.5 & 6 & 0 & 6 & 7.5 & 10897.8180 & 0.0003 \\
\hline 6 & 2 & 4 & 6.5 & 5 & 2 & 3 & 5.5 & 10899.9237 & 0.0004 \\
\hline 6 & 2 & 4 & 5.5 & 5 & 2 & 3 & 4.5 & 10900.0169 & 0.0003 \\
\hline 7 & 0 & 7 & 5.5 & 6 & 0 & 6 & 5.5 & 10903.5420 & -0.0008 \\
\hline 6 & 2 & 4 & 6.5 & 5 & 2 & 3 & 6.5 & 10915.8430 & -0.0032 \\
\hline 7 & 1 & 7 & 7.5 & 6 & 0 & 6 & 7.5 & 11097.2582 & -0.0003 \\
\hline 7 & 1 & 7 & 7.5 & 6 & 0 & 6 & 6.5 & 11102.6196 & 0.0002 \\
\hline 7 & 1 & 7 & 6.5 & 6 & 0 & 6 & 5.5 & 11103.2624 & -0.0002 \\
\hline 7 & 1 & 7 & 6.5 & 6 & 0 & 6 & 6.5 & 11104.5070 & 0.0000 \\
\hline 7 & 1 & 7 & 8.5 & 6 & 0 & 6 & 7.5 & 11106.4998 & 0.0004 \\
\hline 7 & 1 & 7 & 5.5 & 6 & 0 & 6 & 4.5 & 11107.0377 & 0.0000 \\
\hline 3 & 2 & 1 & 1.5 & 2 & 1 & 2 & 0.5 & 11108.5003 & 0.0017 \\
\hline 7 & 1 & 7 & 5.5 & 6 & 0 & 6 & 5.5 & 11112.5410 & -0.0002 \\
\hline 3 & 2 & 1 & 4.5 & 2 & 1 & 2 & 3.5 & 11117.3583 & 0.0023 \\
\hline 3 & 2 & 1 & 1.5 & 2 & 1 & 2 & 1.5 & 11117.5963 & -0.0006 \\
\hline 3 & 2 & 1 & 2.5 & 2 & 1 & 2 & 3.5 & 11120.0184 & 0.0004 \\
\hline 3 & 2 & 1 & 3.5 & 2 & 1 & 2 & 3.5 & 11122.3691 & 0.0001 \\
\hline 3 & 2 & 1 & 2.5 & 2 & 1 & 2 & 1.5 & 11122.5218 & 0.0006 \\
\hline 3 & 2 & 1 & 1.5 & 2 & 1 & 2 & 2.5 & 11124.1214 & -0.0004 \\
\hline 3 & 2 & 1 & 2.5 & 2 & 1 & 2 & 2.5 & 11129.0460 & -0.0002 \\
\hline 3 & 2 & 1 & 3.5 & 2 & 1 & 2 & 2.5 & 11131.3977 & 0.0005 \\
\hline 4 & 2 & 3 & 3.5 & 3 & 1 & 2 & 3.5 & 11470.6667 & -0.0015 \\
\hline 4 & 2 & 3 & 4.5 & 3 & 1 & 2 & 3.5 & 11472.0511 & 0.0001 \\
\hline 4 & 2 & 3 & 2.5 & 3 & 1 & 2 & 2.5 & 11477.0866 & -0.0002 \\
\hline 4 & 2 & 3 & 3.5 & 3 & 1 & 2 & 2.5 & 11479.8969 & -0.0003 \\
\hline 4 & 2 & 3 & 5.5 & 3 & 1 & 2 & 4.5 & 11488.4501 & 0.0010 \\
\hline 4 & 2 & 3 & 4.5 & 3 & 1 & 2 & 4.5 & 11491.7853 & 0.0005 \\
\hline 4 & 2 & 3 & 2.5 & 3 & 1 & 2 & 1.5 & 11496.7209 & 0.0003 \\
\hline 8 & 1 & 7 & 6.5 & 7 & 2 & 6 & 5.5 & 11632.7895 & 0.0005 \\
\hline 8 & 1 & 7 & 9.5 & 7 & 2 & 6 & 8.5 & 11633.9040 & 0.0008 \\
\hline
\end{tabular}


Table S2: Observed Transition Frequencies (in $\mathrm{MHz}$ ) for $\mathrm{CF}_{2} \mathrm{CH}^{35} \mathrm{Cl}-\mathrm{HCCH}$

\begin{tabular}{|c|c|c|c|c|c|c|c|c|c|}
\hline$J^{\prime}$ & $K_{a}^{\prime}$ & $K_{c}^{\prime}$ & $F^{\prime}$ & $J^{\prime \prime}$ & $K_{a}^{\prime \prime}$ & $K_{c}^{\prime \prime}$ & $F^{\prime \prime}$ & Observed & Obs - Calc \\
\hline 8 & 1 & 7 & 7.5 & 7 & 2 & 6 & 6.5 & 11639.6919 & -0.0003 \\
\hline 8 & 1 & 7 & 8.5 & 7 & 2 & 6 & 7.5 & 11640.8225 & -0.0001 \\
\hline 9 & 2 & 8 & 7.5 & 9 & 1 & 9 & 7.5 & 11684.7642 & 0.0013 \\
\hline 9 & 2 & 8 & 10.5 & 9 & 1 & 9 & 10.5 & 11686.7967 & 0.0009 \\
\hline 9 & 2 & 8 & 8.5 & 9 & 1 & 9 & 8.5 & 11697.5287 & 0.0007 \\
\hline 9 & 2 & 8 & 9.5 & 9 & 1 & 9 & 9.5 & 11699.6002 & 0.0011 \\
\hline 7 & 2 & 6 & 5.5 & 6 & 2 & 5 & 5.5 & 11738.9831 & -0.0003 \\
\hline 7 & 2 & 6 & 6.5 & 6 & 2 & 5 & 6.5 & 11741.6769 & 0.0001 \\
\hline 7 & 2 & 6 & 7.5 & 6 & 2 & 5 & 6.5 & 11742.5230 & 0.0004 \\
\hline 7 & 2 & 6 & 6.5 & 6 & 2 & 5 & 5.5 & 11742.7586 & 0.0003 \\
\hline 7 & 2 & 6 & 8.5 & 6 & 2 & 5 & 7.5 & 11742.9920 & 0.0008 \\
\hline 7 & 2 & 6 & 5.5 & 6 & 2 & 5 & 4.5 & 11743.1883 & 0.0004 \\
\hline 7 & 2 & 6 & 7.5 & 6 & 2 & 5 & 7.5 & 11746.8774 & -0.0007 \\
\hline 7 & 5 & 3 & 5.5 & 6 & 5 & 2 & 4.5 & 12066.9583 & -0.0013 \\
\hline 7 & 5 & 3 & 8.5 & 6 & 5 & 2 & 7.5 & 12068.0906 & -0.0010 \\
\hline 7 & 5 & 2 & 8.5 & 6 & 5 & 1 & 7.5 & 12068.4144 & -0.0023 \\
\hline 7 & 5 & 3 & 6.5 & 6 & 5 & 2 & 5.5 & 12071.5408 & 0.0004 \\
\hline 7 & 5 & 2 & 6.5 & 6 & 5 & 1 & 5.5 & 12071.8669 & -0.0007 \\
\hline 7 & 5 & 3 & 7.5 & 6 & 5 & 2 & 6.5 & 12072.6647 & -0.0010 \\
\hline 7 & 5 & 2 & 7.5 & 6 & 5 & 1 & 6.5 & 12072.9959 & -0.0003 \\
\hline 7 & 3 & 5 & 5.5 & 6 & 3 & 4 & 5.5 & 12094.1169 & 0.0000 \\
\hline 8 & 0 & 8 & 8.5 & 7 & 1 & 7 & 8.5 & 12096.8747 & -0.0007 \\
\hline 7 & 3 & 5 & 8.5 & 6 & 3 & 4 & 7.5 & 12099.7951 & 0.0010 \\
\hline 7 & 3 & 5 & 5.5 & 6 & 3 & 4 & 4.5 & 12100.1303 & 0.0015 \\
\hline 7 & 3 & 5 & 6.5 & 6 & 3 & 4 & 6.5 & 12100.3267 & 0.0010 \\
\hline 7 & 3 & 5 & 6.5 & 6 & 3 & 4 & 5.5 & 12101.1555 & 0.0011 \\
\hline 7 & 3 & 5 & 7.5 & 6 & 3 & 4 & 6.5 & 12101.8061 & 0.0008 \\
\hline 7 & 4 & 4 & 5.5 & 6 & 4 & 3 & 4.5 & 12104.2583 & 0.0004 \\
\hline 7 & 4 & 4 & 8.5 & 6 & 4 & 3 & 7.5 & 12104.8638 & 0.0005 \\
\hline 8 & 0 & 8 & 6.5 & 7 & 1 & 7 & 5.5 & 12105.6917 & 0.0002 \\
\hline 8 & 0 & 8 & 7.5 & 7 & 1 & 7 & 6.5 & 12105.8395 & 0.0002 \\
\hline 8 & 0 & 8 & 9.5 & 7 & 1 & 7 & 8.5 & 12105.9324 & 0.0008 \\
\hline 8 & 0 & 8 & 8.5 & 7 & 1 & 7 & 7.5 & 12106.1171 & 0.0009 \\
\hline 7 & 3 & 5 & 7.5 & 6 & 3 & 4 & 7.5 & 12106.9048 & -0.0014 \\
\hline 7 & 4 & 4 & 6.5 & 6 & 4 & 3 & 5.5 & 12107.3787 & 0.0006 \\
\hline 8 & 0 & 8 & 7.5 & 7 & 1 & 7 & 7.5 & 12107.7264 & -0.0005 \\
\hline 7 & 4 & 4 & 7.5 & 6 & 4 & 3 & 6.5 & 12108.0257 & 0.0009 \\
\hline 8 & 0 & 8 & 6.5 & 7 & 1 & 7 & 6.5 & 12114.9705 & 0.0004 \\
\hline 7 & 4 & 3 & 5.5 & 6 & 4 & 2 & 4.5 & 12117.6031 & 0.0013 \\
\hline 7 & 4 & 3 & 8.5 & 6 & 4 & 2 & 7.5 & 12118.1986 & 0.0005 \\
\hline 7 & 4 & 3 & 6.5 & 6 & 4 & 2 & 5.5 & 12120.8521 & 0.0009 \\
\hline 7 & 4 & 3 & 7.5 & 6 & 4 & 2 & 6.5 & 12121.5772 & 0.0013 \\
\hline 8 & 1 & 8 & 8.5 & 7 & 1 & 7 & 8.5 & 12215.4824 & -0.0007 \\
\hline
\end{tabular}


Table S2: Observed Transition Frequencies (in $\mathrm{MHz}$ ) for $\mathrm{CF}_{2} \mathrm{CH}^{35} \mathrm{Cl}-\mathrm{HCCH}$

\begin{tabular}{|c|c|c|c|c|c|c|c|c|c|}
\hline$J^{\prime}$ & $K_{a}{ }^{\prime}$ & $K_{c}{ }^{\prime}$ & $F^{\prime}$ & $J^{\prime \prime}$ & $K_{a}^{\prime \prime}$ & $K_{c}^{\prime \prime}$ & $F^{\prime \prime}$ & Observed & Obs - Calc \\
\hline 8 & 1 & 8 & 7.5 & 7 & 1 & 7 & 6.5 & 12224.6282 & 0.0003 \\
\hline 8 & 1 & 8 & 8.5 & 7 & 1 & 7 & 7.5 & 12224.7247 & 0.0008 \\
\hline 8 & 1 & 8 & 6.5 & 7 & 1 & 7 & 5.5 & 12225.3762 & 0.0007 \\
\hline 8 & 1 & 8 & 9.5 & 7 & 1 & 7 & 8.5 & 12225.4738 & 0.0009 \\
\hline 8 & 1 & 8 & 6.5 & 7 & 1 & 7 & 6.5 & 12234.6559 & 0.0019 \\
\hline 7 & 4 & 3 & 7.5 & 7 & 3 & 4 & 7.5 & 12266.1875 & -0.0003 \\
\hline 7 & 4 & 3 & 6.5 & 7 & 3 & 4 & 6.5 & 12267.6111 & -0.0019 \\
\hline 7 & 4 & 3 & 8.5 & 7 & 3 & 4 & 8.5 & 12273.4133 & 0.0000 \\
\hline 7 & 4 & 3 & 5.5 & 7 & 3 & 4 & 5.5 & 12274.9713 & -0.0017 \\
\hline 8 & 0 & 8 & 8.5 & 7 & 0 & 7 & 8.5 & 12305.5571 & 0.0001 \\
\hline 8 & 0 & 8 & 8.5 & 7 & 0 & 7 & 7.5 & 12313.0578 & 0.0007 \\
\hline 8 & 0 & 8 & 7.5 & 7 & 0 & 7 & 6.5 & 12313.1540 & -0.0001 \\
\hline 8 & 0 & 8 & 9.5 & 7 & 0 & 7 & 8.5 & 12314.6141 & 0.0009 \\
\hline 8 & 0 & 8 & 6.5 & 7 & 0 & 7 & 5.5 & 12314.6894 & -0.0006 \\
\hline 7 & 3 & 4 & 5.5 & 6 & 3 & 3 & 5.5 & 12318.4115 & 0.0012 \\
\hline 8 & 0 & 8 & 6.5 & 7 & 0 & 7 & 6.5 & 12322.2859 & 0.0010 \\
\hline 7 & 3 & 4 & 5.5 & 6 & 3 & 3 & 4.5 & 12326.3147 & 0.0013 \\
\hline 7 & 3 & 4 & 8.5 & 6 & 3 & 3 & 7.5 & 12326.8115 & 0.0003 \\
\hline 7 & 3 & 4 & 6.5 & 6 & 3 & 3 & 6.5 & 12327.8644 & -0.0005 \\
\hline 7 & 3 & 4 & 6.5 & 6 & 3 & 3 & 5.5 & 12329.7410 & 0.0011 \\
\hline 7 & 3 & 4 & 7.5 & 6 & 3 & 3 & 6.5 & 12330.1107 & 0.0009 \\
\hline 7 & 3 & 4 & 7.5 & 6 & 3 & 3 & 7.5 & 12338.0323 & -0.0029 \\
\hline 7 & 1 & 6 & 5.5 & 6 & 1 & 5 & 5.5 & 12379.6736 & 0.0006 \\
\hline 7 & 1 & 6 & 6.5 & 6 & 1 & 5 & 6.5 & 12390.0265 & -0.0005 \\
\hline 7 & 1 & 6 & 7.5 & 6 & 1 & 5 & 6.5 & 12392.4176 & 0.0000 \\
\hline 7 & 1 & 6 & 6.5 & 6 & 1 & 5 & 5.5 & 12393.8870 & 0.0001 \\
\hline 7 & 1 & 6 & 8.5 & 6 & 1 & 5 & 7.5 & 12395.4217 & 0.0009 \\
\hline 7 & 1 & 6 & 5.5 & 6 & 1 & 5 & 4.5 & 12395.9865 & 0.0005 \\
\hline 7 & 1 & 6 & 7.5 & 6 & 1 & 5 & 7.5 & 12408.7362 & -0.0008 \\
\hline 8 & 1 & 8 & 8.5 & 7 & 0 & 7 & 7.5 & 12431.6653 & 0.0005 \\
\hline 8 & 1 & 8 & 7.5 & 7 & 0 & 7 & 6.5 & 12431.9429 & 0.0003 \\
\hline 8 & 1 & 8 & 7.5 & 7 & 0 & 7 & 7.5 & 12433.4553 & -0.0010 \\
\hline 8 & 1 & 8 & 9.5 & 7 & 0 & 7 & 8.5 & 12434.1552 & 0.0007 \\
\hline 8 & 1 & 8 & 6.5 & 7 & 0 & 7 & 5.5 & 12434.3744 & 0.0005 \\
\hline 8 & 1 & 8 & 6.5 & 7 & 0 & 7 & 6.5 & 12441.9711 & 0.0023 \\
\hline 6 & 4 & 2 & 6.5 & 6 & 3 & 3 & 6.5 & 12474.7213 & -0.0004 \\
\hline 6 & 4 & 2 & 5.5 & 6 & 3 & 3 & 5.5 & 12476.4988 & -0.0029 \\
\hline 6 & 4 & 2 & 7.5 & 6 & 3 & 3 & 7.5 & 12482.0282 & 0.0018 \\
\hline 6 & 4 & 2 & 4.5 & 6 & 3 & 3 & 4.5 & 12483.6840 & -0.0008 \\
\hline 5 & 4 & 1 & 5.5 & 5 & 3 & 2 & 5.5 & 12589.1610 & -0.0009 \\
\hline 5 & 4 & 1 & 6.5 & 5 & 3 & 2 & 6.5 & 12597.3705 & 0.0024 \\
\hline 6 & 4 & 3 & 6.5 & 6 & 3 & 4 & 6.5 & 12633.1493 & -0.0006 \\
\hline 6 & 4 & 3 & 5.5 & 6 & 3 & 4 & 5.5 & 12633.8625 & 0.0001 \\
\hline
\end{tabular}


Table S2: Observed Transition Frequencies (in $\mathrm{MHz}$ ) for $\mathrm{CF}_{2} \mathrm{CH}^{35} \mathrm{Cl}-\mathrm{HCCH}$

\begin{tabular}{|c|c|c|c|c|c|c|c|c|c|}
\hline$J^{\prime}$ & $K_{a}^{\prime}$ & $K_{c}^{\prime}$ & $F^{\prime}$ & $J^{\prime \prime}$ & $K_{a}^{\prime \prime}$ & $K_{c}^{\prime \prime}$ & $F^{\prime \prime}$ & Observed & Obs - Calc \\
\hline 6 & 4 & 3 & 7.5 & 6 & 3 & 4 & 7.5 & 12637.6975 & 0.0021 \\
\hline 6 & 4 & 3 & 4.5 & 6 & 3 & 4 & 4.5 & 12639.3065 & -0.0018 \\
\hline 7 & 4 & 4 & 7.5 & 7 & 3 & 5 & 7.5 & 12639.3678 & -0.0016 \\
\hline 7 & 4 & 4 & 6.5 & 7 & 3 & 5 & 6.5 & 12640.0836 & -0.0025 \\
\hline 7 & 4 & 4 & 8.5 & 7 & 3 & 5 & 8.5 & 12642.7653 & 0.0005 \\
\hline 7 & 4 & 4 & 5.5 & 7 & 3 & 5 & 5.5 & 12643.4358 & -0.0015 \\
\hline 4 & 4 & 0 & 4.5 & 4 & 3 & 1 & 4.5 & 12643.9033 & 0.0014 \\
\hline 5 & 4 & 2 & 5.5 & 5 & 3 & 3 & 5.5 & 12644.2554 & -0.0003 \\
\hline 5 & 4 & 2 & 4.5 & 5 & 3 & 3 & 4.5 & 12646.2320 & 0.0010 \\
\hline 4 & 4 & 0 & 3.5 & 4 & 3 & 1 & 3.5 & 12647.7847 & 0.0036 \\
\hline 5 & 2 & 4 & 4.5 & 4 & 1 & 3 & 4.5 & 12648.0885 & -0.0009 \\
\hline 5 & 2 & 4 & 5.5 & 4 & 1 & 3 & 4.5 & 12649.3537 & 0.0009 \\
\hline 5 & 2 & 4 & 3.5 & 4 & 1 & 3 & 3.5 & 12650.8254 & 0.0022 \\
\hline 5 & 4 & 2 & 6.5 & 5 & 3 & 3 & 6.5 & 12651.3236 & 0.0029 \\
\hline 5 & 4 & 2 & 3.5 & 5 & 3 & 3 & 3.5 & 12653.2963 & -0.0006 \\
\hline 4 & 4 & 0 & 5.5 & 4 & 3 & 1 & 5.5 & 12654.8348 & 0.0049 \\
\hline 5 & 2 & 4 & 4.5 & 4 & 1 & 3 & 3.5 & 12654.9322 & 0.0004 \\
\hline 4 & 4 & 1 & 4.5 & 4 & 3 & 2 & 4.5 & 12658.1078 & -0.0003 \\
\hline 4 & 4 & 0 & 2.5 & 4 & 3 & 1 & 2.5 & 12658.5963 & -0.0002 \\
\hline 4 & 4 & 1 & 3.5 & 4 & 3 & 2 & 3.5 & 12661.7569 & -0.0004 \\
\hline 5 & 2 & 4 & 6.5 & 4 & 1 & 3 & 5.5 & 12664.5535 & 0.0018 \\
\hline 4 & 4 & 1 & 5.5 & 4 & 3 & 2 & 5.5 & 12668.5522 & 0.0030 \\
\hline 5 & 2 & 4 & 5.5 & 4 & 1 & 3 & 5.5 & 12668.8151 & -0.0013 \\
\hline 5 & 2 & 4 & 3.5 & 4 & 1 & 3 & 2.5 & 12670.2302 & 0.0004 \\
\hline 4 & 4 & 1 & 2.5 & 4 & 3 & 2 & 2.5 & 12672.2117 & 0.0012 \\
\hline 8 & 4 & 5 & 8.5 & 8 & 3 & 6 & 8.5 & 12682.9772 & 0.0004 \\
\hline 8 & 4 & 5 & 7.5 & 8 & 3 & 6 & 7.5 & 12683.3850 & -0.0008 \\
\hline 8 & 4 & 5 & 9.5 & 8 & 3 & 6 & 9.5 & 12685.0250 & 0.0006 \\
\hline 8 & 4 & 5 & 6.5 & 8 & 3 & 6 & 6.5 & 12685.3351 & -0.0009 \\
\hline 7 & 2 & 5 & 5.5 & 6 & 2 & 4 & 5.5 & 12778.9239 & 0.0014 \\
\hline 7 & 2 & 5 & 6.5 & 6 & 2 & 4 & 6.5 & 12794.6419 & 0.0011 \\
\hline 7 & 2 & 5 & 8.5 & 6 & 2 & 4 & 7.5 & 12797.2708 & 0.0010 \\
\hline 7 & 2 & 5 & 5.5 & 6 & 2 & 4 & 4.5 & 12797.5722 & 0.0011 \\
\hline 7 & 2 & 5 & 7.5 & 6 & 2 & 4 & 6.5 & 12798.7549 & 0.0005 \\
\hline 7 & 2 & 5 & 6.5 & 6 & 2 & 4 & 5.5 & 12799.0684 & 0.0008 \\
\hline 7 & 2 & 5 & 7.5 & 6 & 2 & 4 & 7.5 & 12817.4388 & -0.0009 \\
\hline 8 & 2 & 7 & 6.5 & 7 & 2 & 6 & 6.5 & 13327.5405 & 0.0017 \\
\hline 8 & 2 & 7 & 7.5 & 7 & 2 & 6 & 7.5 & 13329.7058 & -0.0009 \\
\hline 8 & 2 & 7 & 8.5 & 7 & 2 & 6 & 7.5 & 13330.2914 & 0.0004 \\
\hline 8 & 2 & 7 & 7.5 & 7 & 2 & 6 & 6.5 & 13330.5528 & 0.0002 \\
\hline 8 & 2 & 7 & 9.5 & 7 & 2 & 6 & 8.5 & 13331.0779 & 0.0010 \\
\hline 8 & 2 & 7 & 6.5 & 7 & 2 & 6 & 5.5 & 13331.3144 & 0.0007 \\
\hline 8 & 2 & 7 & 8.5 & 7 & 2 & 6 & 8.5 & 13334.1772 & -0.0006 \\
\hline
\end{tabular}


Table S2: Observed Transition Frequencies (in $\mathrm{MHz}$ ) for $\mathrm{CF}_{2} \mathrm{CH}^{35} \mathrm{Cl}-\mathrm{HCCH}$

\begin{tabular}{|c|c|c|c|c|c|c|c|c|c|}
\hline$J^{\prime}$ & $K_{a}^{\prime}$ & $K_{c}^{\prime}$ & $F^{\prime}$ & $J^{\prime \prime}$ & $K_{a}^{\prime \prime}$ & $K_{c}^{\prime \prime}$ & $F^{\prime \prime}$ & Observed & Obs - Calc \\
\hline 4 & 2 & 2 & 2.5 & 3 & 1 & 3 & 1.5 & 13484.9759 & 0.0003 \\
\hline 4 & 2 & 2 & 2.5 & 3 & 1 & 3 & 2.5 & 13492.4077 & 0.0005 \\
\hline 4 & 2 & 2 & 5.5 & 3 & 1 & 3 & 4.5 & 13492.5051 & 0.0017 \\
\hline 4 & 2 & 2 & 3.5 & 3 & 1 & 3 & 2.5 & 13503.8945 & 0.0010 \\
\hline 4 & 2 & 2 & 4.5 & 3 & 1 & 3 & 4.5 & 13504.0400 & -0.0015 \\
\hline 4 & 2 & 2 & 3.5 & 3 & 1 & 3 & 3.5 & 13507.3677 & 0.0003 \\
\hline 4 & 2 & 2 & 4.5 & 3 & 1 & 3 & 3.5 & 13511.4249 & 0.0016 \\
\hline 9 & 0 & 9 & 8.5 & 8 & 1 & 8 & 7.5 & 13617.8869 & 0.0003 \\
\hline 9 & 0 & 9 & 9.5 & 8 & 1 & 8 & 8.5 & 13618.0586 & 0.0002 \\
\hline 9 & 0 & 9 & 7.5 & 8 & 1 & 8 & 6.5 & 13618.1207 & 0.0015 \\
\hline 9 & 0 & 9 & 10.5 & 8 & 1 & 8 & 9.5 & 13618.2642 & -0.0001 \\
\hline 9 & 1 & 9 & 9.5 & 8 & 1 & 8 & 9.5 & 13674.1090 & -0.0021 \\
\hline 9 & 1 & 9 & 8.5 & 8 & 1 & 8 & 7.5 & 13684.0168 & 0.0002 \\
\hline 9 & 1 & 9 & 9.5 & 8 & 1 & 8 & 8.5 & 13684.1011 & 0.0001 \\
\hline 9 & 1 & 9 & 7.5 & 8 & 1 & 8 & 6.5 & 13684.7104 & 0.0004 \\
\hline 9 & 1 & 9 & 10.5 & 8 & 1 & 8 & 9.5 & 13684.7938 & 0.0003 \\
\hline 9 & 1 & 9 & 8.5 & 8 & 1 & 8 & 8.5 & 13685.8088 & 0.0007 \\
\hline 9 & 1 & 9 & 7.5 & 8 & 1 & 8 & 7.5 & 13694.7376 & 0.0014 \\
\hline 6 & 2 & 5 & 5.5 & 5 & 1 & 4 & 5.5 & 13711.4753 & 0.0022 \\
\hline 6 & 2 & 5 & 4.5 & 5 & 1 & 4 & 4.5 & 13712.4298 & -0.0019 \\
\hline 6 & 2 & 5 & 6.5 & 5 & 1 & 4 & 5.5 & 13712.5557 & 0.0012 \\
\hline 9 & 1 & 8 & 7.5 & 8 & 2 & 7 & 6.5 & 13712.8847 & 0.0017 \\
\hline 9 & 1 & 8 & 10.5 & 8 & 2 & 7 & 9.5 & 13713.5453 & 0.0000 \\
\hline 6 & 2 & 5 & 5.5 & 5 & 1 & 4 & 4.5 & 13716.6363 & 0.0002 \\
\hline 9 & 1 & 8 & 8.5 & 8 & 2 & 7 & 7.5 & 13717.4297 & -0.0002 \\
\hline 9 & 1 & 8 & 9.5 & 8 & 2 & 7 & 8.5 & 13718.0996 & -0.0006 \\
\hline 6 & 2 & 5 & 7.5 & 5 & 1 & 4 & 6.5 & 13726.4628 & 0.0016 \\
\hline 9 & 0 & 9 & 9.5 & 8 & 0 & 8 & 9.5 & 13727.6065 & -0.0035 \\
\hline 6 & 2 & 5 & 4.5 & 5 & 1 & 4 & 3.5 & 13730.6557 & 0.0001 \\
\hline 6 & 2 & 5 & 6.5 & 5 & 1 & 4 & 6.5 & 13730.8166 & -0.0001 \\
\hline 9 & 0 & 9 & 9.5 & 8 & 0 & 8 & 8.5 & 13736.6639 & -0.0023 \\
\hline 9 & 0 & 9 & 8.5 & 8 & 0 & 8 & 7.5 & 13736.6785 & 0.0033 \\
\hline 9 & 0 & 9 & 7.5 & 8 & 0 & 8 & 6.5 & \multirow{2}{*}{13737.8053} & \multirow{2}{*}{0.0009} \\
\hline 9 & 0 & 9 & 10.5 & 8 & 0 & 8 & 9.5 & & \\
\hline 9 & 0 & 9 & 8.5 & 8 & 0 & 8 & 8.5 & 13738.2844 & -0.0015 \\
\hline 9 & 0 & 9 & 7.5 & 8 & 0 & 8 & 7.5 & 13746.9348 & 0.0008 \\
\hline 9 & 1 & 9 & 9.5 & 8 & 0 & 8 & 8.5 & 13802.7095 & 0.0008 \\
\hline 9 & 1 & 9 & 8.5 & 8 & 0 & 8 & 7.5 & 13802.8055 & 0.0004 \\
\hline 9 & 1 & 9 & 10.5 & 8 & 0 & 8 & 9.5 & 13804.3343 & -0.0006 \\
\hline 9 & 1 & 9 & 7.5 & 8 & 0 & 8 & 6.5 & 13804.3966 & 0.0026 \\
\hline 8 & 5 & 4 & 6.5 & 7 & 5 & 3 & 5.5 & 13816.1409 & 0.0012 \\
\hline 8 & 5 & 4 & 9.5 & 7 & 5 & 3 & 8.5 & 13816.7697 & 0.0007 \\
\hline 8 & 5 & 3 & 6.5 & 7 & 5 & 2 & 5.5 & 13817.4158 & 0.0001 \\
\hline
\end{tabular}


Table S2: Observed Transition Frequencies (in $\mathrm{MHz}$ ) for $\mathrm{CF}_{2} \mathrm{CH}^{35} \mathrm{Cl}-\mathrm{HCCH}$

\begin{tabular}{|c|c|c|c|c|c|c|c|c|c|}
\hline$J^{\prime}$ & $K_{a}^{\prime}$ & $K_{c}{ }^{\prime}$ & $F^{\prime}$ & $J^{\prime \prime}$ & $K_{a}^{\prime \prime}$ & $K_{c}^{\prime \prime}$ & $F^{\prime \prime}$ & Observed & Obs - Calc \\
\hline 8 & 5 & 3 & 9.5 & 7 & 5 & 2 & 8.5 & 13818.0496 & -0.0012 \\
\hline 8 & 5 & 3 & 7.5 & 7 & 5 & 2 & 6.5 & 13820.6499 & -0.0014 \\
\hline 8 & 5 & 3 & 8.5 & 7 & 5 & 2 & 7.5 & 13821.2390 & 0.0003 \\
\hline 8 & 3 & 6 & 9.5 & 7 & 3 & 5 & 8.5 & 13822.4844 & 0.0018 \\
\hline 8 & 3 & 6 & 6.5 & 7 & 3 & 5 & 5.5 & 13822.5178 & 0.0000 \\
\hline 8 & 3 & 6 & 8.5 & 7 & 3 & 5 & 7.5 & 13823.3456 & -0.0001 \\
\hline 8 & 3 & 6 & 7.5 & 7 & 3 & 5 & 6.5 & 13823.3646 & -0.0007 \\
\hline 8 & 4 & 5 & 6.5 & 7 & 4 & 4 & 5.5 & 13864.4173 & 0.0009 \\
\hline 8 & 4 & 5 & 9.5 & 7 & 4 & 4 & 8.5 & 13864.7430 & 0.0007 \\
\hline 8 & 4 & 5 & 7.5 & 7 & 4 & 4 & 6.5 & 13866.6657 & 0.0008 \\
\hline 8 & 4 & 5 & 8.5 & 7 & 4 & 4 & 7.5 & 13866.9542 & 0.0011 \\
\hline 8 & 4 & 4 & 6.5 & 7 & 4 & 3 & 5.5 & 13900.2960 & 0.0011 \\
\hline 8 & 4 & 4 & 9.5 & 7 & 4 & 3 & 8.5 & 13900.6696 & 0.0014 \\
\hline 8 & 4 & 4 & 7.5 & 7 & 4 & 3 & 6.5 & 13902.8892 & 0.0008 \\
\hline 8 & 4 & 4 & 8.5 & 7 & 4 & 3 & 7.5 & 13903.2461 & 0.0010 \\
\hline 8 & 1 & 7 & 6.5 & 7 & 1 & 6 & 6.5 & 13929.0640 & 0.0020 \\
\hline 8 & 1 & 7 & 7.5 & 7 & 1 & 6 & 7.5 & 13937.3497 & 0.0001 \\
\hline 8 & 1 & 7 & 8.5 & 7 & 1 & 6 & 7.5 & 13939.3257 & 0.0001 \\
\hline 8 & 1 & 7 & 7.5 & 7 & 1 & 6 & 6.5 & 13939.7400 & -0.0002 \\
\hline 8 & 1 & 7 & 9.5 & 7 & 1 & 6 & 8.5 & 13941.8359 & 0.0004 \\
\hline 8 & 1 & 7 & 6.5 & 7 & 1 & 6 & 5.5 & 13943.2760 & 0.0001 \\
\hline 8 & 1 & 7 & 8.5 & 7 & 1 & 6 & 8.5 & 13952.6381 & -0.0037 \\
\hline 3 & 3 & 1 & 3.5 & 2 & 2 & 0 & 3.5 & 14188.5170 & -0.0027 \\
\hline 3 & 3 & 1 & 3.5 & 2 & 2 & 0 & 2.5 & 14195.4854 & 0.0005 \\
\hline 3 & 3 & 1 & 2.5 & 2 & 2 & 0 & 1.5 & 14195.5232 & -0.0018 \\
\hline 3 & 3 & 1 & 1.5 & 2 & 2 & 0 & 0.5 & 14199.1836 & 0.0004 \\
\hline 3 & 3 & 1 & 4.5 & 2 & 2 & 0 & 3.5 & 14199.2350 & 0.0019 \\
\hline 3 & 3 & 1 & 2.5 & 2 & 2 & 0 & 2.5 & 14200.4895 & -0.0020 \\
\hline 3 & 3 & 1 & 1.5 & 2 & 2 & 0 & 1.5 & 14206.2662 & 0.0035 \\
\hline 3 & 3 & 0 & 2.5 & 2 & 2 & 1 & 1.5 & 14226.7490 & -0.0001 \\
\hline 3 & 3 & 0 & 3.5 & 2 & 2 & 1 & 2.5 & 14228.2921 & 0.0004 \\
\hline 3 & 3 & 0 & 1.5 & 2 & 2 & 1 & 0.5 & 14228.3437 & 0.0046 \\
\hline 3 & 3 & 0 & 4.5 & 2 & 2 & 1 & 3.5 & 14229.8524 & 0.0018 \\
\hline 3 & 3 & 0 & 2.5 & 2 & 2 & 1 & 2.5 & 14233.2453 & -0.0025 \\
\hline 8 & 3 & 5 & 6.5 & 7 & 3 & 4 & 5.5 & 14237.2908 & -0.0016 \\
\hline 8 & 3 & 5 & 9.5 & 7 & 3 & 4 & 8.5 & 14237.4950 & 0.0007 \\
\hline 8 & 3 & 5 & 7.5 & 7 & 3 & 4 & 6.5 & 14240.3183 & 0.0012 \\
\hline 8 & 3 & 5 & 8.5 & 7 & 3 & 4 & 7.5 & 14240.6529 & 0.0006 \\
\hline 8 & 2 & 6 & 6.5 & 7 & 2 & 5 & 6.5 & 14635.2397 & 0.0022 \\
\hline 8 & 2 & 6 & 7.5 & 7 & 2 & 5 & 7.5 & 14651.6855 & -0.0005 \\
\hline 8 & 2 & 6 & 9.5 & 7 & 2 & 5 & 8.5 & 14654.9654 & 0.0007 \\
\hline 8 & 2 & 6 & 8.5 & 7 & 2 & 5 & 7.5 & 14655.3708 & -0.0017 \\
\hline 8 & 2 & 6 & 6.5 & 7 & 2 & 5 & 5.5 & 14655.3868 & 0.0043 \\
\hline
\end{tabular}


Table S2: Observed Transition Frequencies (in $\mathrm{MHz}$ ) for $\mathrm{CF}_{2} \mathrm{CH}^{35} \mathrm{Cl}-\mathrm{HCCH}$

\begin{tabular}{|c|c|c|c|c|c|c|c|c|c|}
\hline$J^{\prime}$ & $K_{a}^{\prime}$ & $K_{c}^{\prime}$ & $F^{\prime}$ & $J^{\prime \prime}$ & $K_{a}^{\prime \prime}$ & $K_{c}^{\prime \prime}$ & $F^{\prime \prime}$ & Observed & Obs - Calc \\
\hline 8 & 2 & 6 & 7.5 & 7 & 2 & 5 & 6.5 & 14655.7997 & 0.0001 \\
\hline 8 & 2 & 6 & 8.5 & 7 & 2 & 5 & 8.5 & 14675.5399 & -0.0024 \\
\hline 7 & 2 & 6 & 7.5 & 6 & 1 & 5 & 6.5 & 14690.9214 & 0.0007 \\
\hline 7 & 2 & 6 & 6.5 & 6 & 1 & 5 & 5.5 & 14693.9347 & -0.0002 \\
\hline 7 & 2 & 6 & 8.5 & 6 & 1 & 5 & 7.5 & 14703.3547 & 0.0016 \\
\hline 7 & 2 & 6 & 5.5 & 6 & 1 & 5 & 4.5 & 14706.4728 & 0.0000 \\
\hline 9 & 2 & 8 & 9.5 & 8 & 2 & 7 & 8.5 & 14888.9810 & 0.0004 \\
\hline 9 & 2 & 8 & 8.5 & 8 & 2 & 7 & 7.5 & 14889.2013 & 0.0004 \\
\hline 9 & 2 & 8 & 10.5 & 8 & 2 & 7 & 9.5 & 14889.9615 & 0.0009 \\
\hline 9 & 2 & 8 & 7.5 & 8 & 2 & 7 & 6.5 & 14890.1691 & 0.0001 \\
\hline 10 & 0 & 10 & 9.5 & 9 & 1 & 9 & 8.5 & 15100.5203 & -0.0002 \\
\hline 10 & 0 & 10 & 10.5 & 9 & 1 & 9 & 9.5 & 15100.6415 & 0.0001 \\
\hline 10 & 0 & 10 & 8.5 & 9 & 1 & 9 & 7.5 & 15100.9100 & -0.0007 \\
\hline 10 & 0 & 10 & 11.5 & 9 & 1 & 9 & 10.5 & 15101.0127 & -0.0003 \\
\hline 10 & 1 & 10 & 9.5 & 9 & 1 & 9 & 8.5 & 15136.5343 & 0.0009 \\
\hline 10 & 1 & 10 & 10.5 & 9 & 1 & 9 & 9.5 & 15136.6113 & -0.0004 \\
\hline 10 & 1 & 10 & 8.5 & 9 & 1 & 9 & 7.5 & 15137.1564 & 0.0009 \\
\hline 10 & 1 & 10 & 11.5 & 9 & 1 & 9 & 10.5 & 15137.2320 & -0.0002 \\
\hline 10 & 0 & 10 & 9.5 & 9 & 0 & 9 & 8.5 & 15166.6494 & -0.0011 \\
\hline 10 & 0 & 10 & 10.5 & 9 & 0 & 9 & 9.5 & 15166.6835 & -0.0004 \\
\hline 10 & 0 & 10 & 8.5 & 9 & 0 & 9 & 7.5 & 15167.5036 & 0.0022 \\
\hline 10 & 0 & 10 & 11.5 & 9 & 0 & 9 & 10.5 & 15167.5427 & 0.0005 \\
\hline 10 & 1 & 10 & 10.5 & 9 & 0 & 9 & 9.5 & 15202.6507 & -0.0036 \\
\hline 10 & 1 & 10 & 9.5 & 9 & 0 & 9 & 8.5 & 15202.6672 & 0.0039 \\
\hline 10 & 1 & 10 & 8.5 & 9 & 0 & 9 & 7.5 & 15203.7458 & -0.0005 \\
\hline 10 & 1 & 10 & 11.5 & 9 & 0 & 9 & 10.5 & 15203.7634 & 0.0020 \\
\hline 9 & 1 & 8 & 9.5 & 8 & 1 & 7 & 8.5 & 15407.5689 & 0.0003 \\
\hline 9 & 1 & 8 & 8.5 & 8 & 1 & 7 & 7.5 & 15408.2899 & -0.0002 \\
\hline 9 & 1 & 8 & 10.5 & 8 & 1 & 7 & 9.5 & 15410.7194 & 0.0005 \\
\hline 9 & 1 & 8 & 7.5 & 8 & 1 & 7 & 6.5 & 15411.4076 & -0.0002 \\
\hline 9 & 3 & 7 & 10.5 & 8 & 3 & 6 & 9.5 & 15525.5835 & 0.0009 \\
\hline 9 & 3 & 7 & 7.5 & 8 & 3 & 6 & 6.5 & 15525.6722 & 0.0007 \\
\hline 9 & 3 & 7 & 9.5 & 8 & 3 & 6 & 8.5 & 15525.9073 & -0.0001 \\
\hline 9 & 3 & 7 & 8.5 & 8 & 3 & 6 & 7.5 & 15526.0232 & 0.0005 \\
\hline 9 & 5 & 5 & 7.5 & 8 & 5 & 4 & 6.5 & 15573.9793 & -0.0013 \\
\hline 9 & 5 & 5 & 10.5 & 8 & 5 & 4 & 9.5 & 15574.3066 & -0.0004 \\
\hline 9 & 5 & 5 & 8.5 & 8 & 5 & 4 & 7.5 & 15576.3273 & 0.0010 \\
\hline 9 & 5 & 5 & 9.5 & 8 & 5 & 4 & 8.5 & 15576.6981 & -0.0007 \\
\hline 9 & 5 & 4 & 7.5 & 8 & 5 & 3 & 6.5 & 15578.0723 & -0.0011 \\
\hline 9 & 5 & 4 & 10.5 & 8 & 5 & 3 & 9.5 & 15578.4011 & -0.0008 \\
\hline 9 & 5 & 4 & 8.5 & 8 & 5 & 3 & 7.5 & 15580.4594 & 0.0015 \\
\hline 9 & 5 & 4 & 9.5 & 8 & 5 & 3 & 8.5 & 15580.8413 & -0.0006 \\
\hline 8 & 2 & 7 & 8.5 & 7 & 1 & 6 & 7.5 & 15628.7945 & 0.0004 \\
\hline
\end{tabular}


Table S2: Observed Transition Frequencies (in $\mathrm{MHz}$ ) for $\mathrm{CF}_{2} \mathrm{CH}^{35} \mathrm{Cl}-\mathrm{HCCH}$

\begin{tabular}{|c|c|c|c|c|c|c|c|c|c|}
\hline$J^{\prime}$ & $K_{a}^{\prime}$ & $K_{c}^{\prime}$ & $F^{\prime}$ & $J^{\prime \prime}$ & $K_{a}^{\prime \prime}$ & $K_{c}^{\prime \prime}$ & $F^{\prime \prime}$ & Observed & Obs - Calc \\
\hline 9 & 4 & 6 & 7.5 & 8 & 4 & 5 & 6.5 & 15629.6095 & 0.0015 \\
\hline 8 & 2 & 7 & 7.5 & 7 & 1 & 6 & 6.5 & 15630.6004 & 0.0000 \\
\hline 9 & 4 & 6 & 9.5 & 8 & 4 & 5 & 8.5 & 15631.4513 & 0.0012 \\
\hline 9 & 4 & 6 & 10.5 & 8 & 4 & 5 & 9.5 & 15632.1693 & -0.0002 \\
\hline 9 & 4 & 6 & 8.5 & 8 & 4 & 5 & 7.5 & 15632.4097 & 0.0005 \\
\hline 8 & 2 & 7 & 9.5 & 7 & 1 & 6 & 8.5 & 15639.0093 & 0.0001 \\
\hline 8 & 2 & 7 & 6.5 & 7 & 1 & 6 & 5.5 & 15641.8005 & -0.0002 \\
\hline 9 & 4 & 5 & 7.5 & 8 & 4 & 4 & 6.5 & 15712.6837 & 0.0019 \\
\hline 9 & 4 & 5 & 10.5 & 8 & 4 & 4 & 9.5 & 15712.9443 & -0.0003 \\
\hline 9 & 4 & 5 & 8.5 & 8 & 4 & 4 & 7.5 & 15714.9761 & 0.0002 \\
\hline 9 & 4 & 5 & 9.5 & 8 & 4 & 4 & 8.5 & 15715.1907 & 0.0004 \\
\hline 4 & 3 & 2 & 4.5 & 3 & 2 & 1 & 3.5 & 15840.7633 & -0.0005 \\
\hline 4 & 3 & 2 & 3.5 & 3 & 2 & 1 & 3.5 & 15841.3901 & -0.0011 \\
\hline 4 & 3 & 2 & 3.5 & 3 & 2 & 1 & 2.5 & 15843.7416 & -0.0007 \\
\hline 4 & 3 & 2 & 2.5 & 3 & 2 & 1 & 2.5 & 15845.4973 & 0.0002 \\
\hline 4 & 3 & 2 & 4.5 & 3 & 2 & 1 & 4.5 & 15845.7755 & -0.0013 \\
\hline 4 & 3 & 2 & 5.5 & 3 & 2 & 1 & 4.5 & 15847.5240 & 0.0009 \\
\hline 4 & 3 & 2 & 2.5 & 3 & 2 & 1 & 1.5 & 15850.4219 & 0.0005 \\
\hline 4 & 3 & 1 & 3.5 & 3 & 2 & 2 & 2.5 & 16001.5198 & 0.0005 \\
\hline 4 & 3 & 1 & 4.5 & 3 & 2 & 2 & 4.5 & 16001.9811 & -0.0023 \\
\hline 4 & 3 & 1 & 4.5 & 3 & 2 & 2 & 3.5 & 16002.3005 & -0.0001 \\
\hline 4 & 3 & 1 & 3.5 & 3 & 2 & 2 & 3.5 & 16002.6958 & -0.0004 \\
\hline 4 & 3 & 1 & 2.5 & 3 & 2 & 2 & 2.5 & 16002.9060 & -0.0010 \\
\hline 4 & 3 & 1 & 5.5 & 3 & 2 & 2 & 4.5 & 16003.2386 & 0.0006 \\
\hline 4 & 3 & 1 & 2.5 & 3 & 2 & 2 & 1.5 & 16004.2044 & 0.0002 \\
\hline 9 & 3 & 6 & 7.5 & 8 & 3 & 5 & 6.5 & 16194.3782 & 0.0006 \\
\hline 9 & 3 & 6 & 10.5 & 8 & 3 & 5 & 9.5 & 16194.5432 & 0.0007 \\
\hline 9 & 3 & 6 & 8.5 & 8 & 3 & 5 & 7.5 & 16197.1189 & 0.0005 \\
\hline 9 & 3 & 6 & 9.5 & 8 & 3 & 5 & 8.5 & 16197.2744 & 0.0007 \\
\hline 7 & 5 & 2 & 7.5 & 7 & 4 & 3 & 7.5 & 16199.8242 & -0.0023 \\
\hline 7 & 5 & 2 & 6.5 & 7 & 4 & 3 & 6.5 & 16200.9515 & -0.0011 \\
\hline 7 & 5 & 2 & 8.5 & 7 & 4 & 3 & 8.5 & 16205.3302 & 0.0012 \\
\hline 7 & 5 & 2 & 5.5 & 7 & 4 & 3 & 5.5 & 16206.4425 & -0.0020 \\
\hline 5 & 2 & 3 & 3.5 & 4 & 1 & 4 & 2.5 & 16216.6023 & -0.0014 \\
\hline 7 & 5 & 3 & 7.5 & 7 & 4 & 4 & 7.5 & 16218.1808 & -0.0009 \\
\hline 7 & 5 & 3 & 6.5 & 7 & 4 & 4 & 6.5 & 16219.2526 & -0.0020 \\
\hline 5 & 2 & 3 & 6.5 & 4 & 1 & 4 & 5.5 & 16222.4097 & 0.0004 \\
\hline 7 & 5 & 3 & 8.5 & 7 & 4 & 4 & 8.5 & 16223.4092 & -0.0003 \\
\hline 7 & 5 & 3 & 5.5 & 7 & 4 & 4 & 5.5 & 16224.4686 & -0.0021 \\
\hline 5 & 2 & 3 & 4.5 & 4 & 1 & 4 & 3.5 & 16238.6631 & 0.0014 \\
\hline 5 & 2 & 3 & 5.5 & 4 & 1 & 4 & 4.5 & 16246.0503 & 0.0014 \\
\hline 6 & 5 & 1 & 5.5 & 6 & 4 & 2 & 5.5 & 16249.9370 & 0.0008 \\
\hline 6 & 5 & 2 & 6.5 & 6 & 4 & 3 & 6.5 & 16253.5385 & -0.0021 \\
\hline
\end{tabular}


Table S2: Observed Transition Frequencies (in $\mathrm{MHz}$ ) for $\mathrm{CF}_{2} \mathrm{CH}^{35} \mathrm{Cl}-\mathrm{HCCH}$

\begin{tabular}{|c|c|c|c|c|c|c|c|c|c|}
\hline$J^{\prime}$ & $K_{a}{ }^{\prime}$ & $K_{c}{ }^{\prime}$ & $F^{\prime}$ & $J^{\prime \prime}$ & $K_{a}^{\prime \prime}$ & $K_{c}^{\prime \prime}$ & $F^{\prime \prime}$ & Observed & Obs - Calc \\
\hline 6 & 5 & 2 & 5.5 & 6 & 4 & 3 & 5.5 & 16255.0932 & 0.0011 \\
\hline 6 & 5 & 1 & 7.5 & 6 & 4 & 2 & 7.5 & 16255.1132 & 0.0028 \\
\hline 6 & 5 & 2 & 7.5 & 6 & 4 & 3 & 7.5 & 16260.1847 & 0.0035 \\
\hline 6 & 5 & 2 & 4.5 & 6 & 4 & 3 & 4.5 & 16261.7679 & -0.0012 \\
\hline 10 & 2 & 9 & 10.5 & 9 & 2 & 8 & 9.5 & 16420.1417 & 0.0002 \\
\hline 10 & 2 & 9 & 9.5 & 9 & 2 & 8 & 8.5 & 16420.3328 & -0.0004 \\
\hline 10 & 2 & 9 & 11.5 & 9 & 2 & 8 & 10.5 & 16421.2250 & 0.0002 \\
\hline 10 & 2 & 9 & 8.5 & 9 & 2 & 8 & 7.5 & 16421.4089 & 0.0001 \\
\hline 9 & 2 & 7 & 9.5 & 8 & 2 & 6 & 8.5 & 16451.6664 & -0.0010 \\
\hline 9 & 2 & 7 & 8.5 & 8 & 2 & 6 & 7.5 & \multirow{2}{*}{16452.1492} & \multirow{2}{*}{0.0000} \\
\hline 9 & 2 & 7 & 10.5 & 8 & 2 & 6 & 9.5 & & \\
\hline 9 & 2 & 7 & 7.5 & 8 & 2 & 6 & 6.5 & 16452.6120 & 0.0002 \\
\hline 9 & 2 & 8 & 9.5 & 8 & 1 & 7 & 8.5 & 16578.4500 & 0.0010 \\
\hline 9 & 2 & 8 & 8.5 & 8 & 1 & 7 & 7.5 & 16580.0624 & 0.0012 \\
\hline 11 & 1 & 11 & 10.5 & 10 & 1 & 10 & 9.5 & 16584.6403 & 0.0036 \\
\hline 11 & 1 & 11 & 11.5 & 10 & 1 & 10 & 10.5 & 16584.7071 & -0.0036 \\
\hline 11 & 1 & 11 & 12.5 & 10 & 1 & 10 & 11.5 & 16585.2567 & -0.0005 \\
\hline 9 & 2 & 8 & 10.5 & 8 & 1 & 7 & 9.5 & 16587.1356 & 0.0013 \\
\hline 9 & 2 & 8 & 7.5 & 8 & 1 & 7 & 6.5 & 16588.6939 & 0.0001 \\
\hline 11 & 0 & 11 & 10.5 & 10 & 0 & 10 & 9.5 & 16601.3757 & 0.0014 \\
\hline 11 & 0 & 11 & 11.5 & 10 & 0 & 10 & 10.5 & 16601.4248 & -0.0017 \\
\hline 11 & 0 & 11 & 9.5 & 10 & 0 & 10 & 8.5 & 16602.0379 & -0.0012 \\
\hline 11 & 0 & 11 & 12.5 & 10 & 0 & 10 & 11.5 & 16602.0973 & 0.0007 \\
\hline 10 & 1 & 9 & 10.5 & 9 & 1 & 8 & 9.5 & 16822.4049 & 0.0010 \\
\hline 10 & 1 & 9 & 9.5 & 9 & 1 & 8 & 8.5 & 16822.9476 & -0.0003 \\
\hline 10 & 1 & 9 & 11.5 & 9 & 1 & 8 & 10.5 & 16825.3330 & -0.0003 \\
\hline 10 & 1 & 9 & 8.5 & 9 & 1 & 8 & 7.5 & 16825.8672 & 0.0000 \\
\hline 10 & 3 & 8 & 10.5 & 9 & 3 & 7 & 9.5 & 17203.4690 & -0.0017 \\
\hline 10 & 3 & 8 & 11.5 & 9 & 3 & 7 & 10.5 & 17203.5450 & 0.0030 \\
\hline 10 & 3 & 8 & 9.5 & 9 & 3 & 7 & 8.5 & 17203.6089 & -0.0020 \\
\hline 10 & 3 & 8 & 8.5 & 9 & 3 & 7 & 7.5 & 17203.6725 & 0.0011 \\
\hline 5 & 3 & 3 & 4.5 & 4 & 2 & 2 & 4.5 & 17378.6216 & -0.0004 \\
\hline 5 & 3 & 3 & 5.5 & 4 & 2 & 2 & 4.5 & 17379.4290 & -0.0001 \\
\hline 5 & 3 & 3 & 3.5 & 4 & 2 & 2 & 3.5 & 17379.8282 & -0.0005 \\
\hline 5 & 3 & 3 & 4.5 & 4 & 2 & 2 & 3.5 & 17382.6776 & -0.0004 \\
\hline 5 & 3 & 3 & 6.5 & 4 & 2 & 2 & 5.5 & 17388.0969 & 0.0013 \\
\hline 5 & 3 & 3 & 5.5 & 4 & 2 & 2 & 5.5 & 17390.9637 & -0.0035 \\
\hline 5 & 3 & 3 & 3.5 & 4 & 2 & 2 & 2.5 & 17391.3147 & -0.0003 \\
\hline 10 & 4 & 7 & 11.5 & 9 & 4 & 6 & 10.5 & 17393.0748 & 0.0016 \\
\hline 10 & 4 & 7 & 9.5 & 9 & 4 & 6 & 8.5 & 17395.4617 & 0.0016 \\
\hline 10 & 4 & 7 & 10.5 & 9 & 4 & 6 & 9.5 & 17396.6690 & -0.0001 \\
\hline 10 & 4 & 6 & 8.5 & 9 & 4 & 5 & 7.5 & 17566.0395 & 0.0009 \\
\hline 10 & 4 & 6 & 11.5 & 9 & 4 & 5 & 10.5 & 17566.1886 & 0.0011 \\
\hline
\end{tabular}


Table S2: Observed Transition Frequencies (in $\mathrm{MHz}$ ) for $\mathrm{CF}_{2} \mathrm{CH}^{35} \mathrm{Cl}-\mathrm{HCCH}$

\begin{tabular}{|c|c|c|c|c|c|c|c|c|c|}
\hline$J^{\prime}$ & $K_{a}^{\prime}$ & $K_{c}^{\prime}$ & $F^{\prime}$ & $J^{\prime \prime}$ & $K_{a}^{\prime \prime}$ & $K_{c}^{\prime \prime}$ & $F^{\prime \prime}$ & Observed & Obs - Calc \\
\hline 10 & 4 & 6 & 9.5 & 9 & 4 & 5 & 8.5 & 17568.2102 & 0.0006 \\
\hline 10 & 4 & 6 & 10.5 & 9 & 4 & 5 & 9.5 & 17568.4169 & 0.0004 \\
\hline 5 & 3 & 2 & 3.5 & 4 & 2 & 3 & 3.5 & 17854.6491 & 0.0004 \\
\hline 5 & 3 & 2 & 4.5 & 4 & 2 & 3 & 4.5 & 17857.2732 & 0.0006 \\
\hline 5 & 3 & 2 & 3.5 & 4 & 2 & 3 & 2.5 & 17857.4592 & 0.0000 \\
\hline 5 & 3 & 2 & 6.5 & 4 & 2 & 3 & 5.5 & 17857.7098 & 0.0012 \\
\hline 5 & 3 & 2 & 5.5 & 4 & 2 & 3 & 4.5 & 17858.4200 & -0.0004 \\
\hline 5 & 3 & 2 & 4.5 & 4 & 2 & 3 & 3.5 & 17858.6542 & -0.0011 \\
\hline 5 & 3 & 2 & 5.5 & 4 & 2 & 3 & 5.5 & 17861.7552 & -0.0009 \\
\hline 11 & 2 & 10 & 11.5 & 10 & 2 & 9 & 10.5 & 17926.9603 & 0.0003 \\
\hline 11 & 2 & 10 & 10.5 & 10 & 2 & 9 & 9.5 & 17927.1211 & -0.0005 \\
\hline 11 & 2 & 10 & 12.5 & 10 & 2 & 9 & 11.5 & 17928.0768 & -0.0004 \\
\hline 11 & 2 & 10 & 9.5 & 10 & 2 & 9 & 8.5 & 17928.2339 & -0.0004 \\
\hline 12 & 1 & 12 & 11.5 & 11 & 1 & 11 & 10.5 & 18030.0347 & 0.0005 \\
\hline 12 & 1 & 12 & 12.5 & 11 & 1 & 11 & 11.5 & 18030.1038 & -0.0004 \\
\hline 12 & 1 & 12 & 10.5 & 11 & 1 & 11 & 9.5 & 18030.5132 & 0.0006 \\
\hline 12 & 1 & 12 & 13.5 & 11 & 1 & 11 & 12.5 & 18030.5810 & -0.0011 \\
\hline 12 & 0 & 12 & 11.5 & 11 & 0 & 11 & 10.5 & 18039.1351 & -0.0006 \\
\hline 12 & 0 & 12 & 12.5 & 11 & 0 & 11 & 11.5 & 18039.1933 & -0.0019 \\
\hline 12 & 0 & 12 & 10.5 & 11 & 0 & 11 & 9.5 & 18039.6712 & -0.0017 \\
\hline 12 & 0 & 12 & 13.5 & 11 & 0 & 11 & 12.5 & 18039.7363 & 0.0004 \\
\hline 10 & 3 & 7 & 8.5 & 9 & 3 & 6 & 7.5 & 18169.3164 & -0.0002 \\
\hline 10 & 3 & 7 & 11.5 & 9 & 3 & 6 & 10.5 & 18169.3644 & 0.0001 \\
\hline 10 & 2 & 8 & 10.5 & 9 & 2 & 7 & 9.5 & 18170.8745 & 0.0004 \\
\hline 10 & 3 & 7 & 9.5 & 9 & 3 & 6 & 8.5 & 18171.4585 & -0.0024 \\
\hline 10 & 3 & 7 & 10.5 & 9 & 3 & 6 & 9.5 & 18171.5034 & -0.0001 \\
\hline 10 & 2 & 8 & 9.5 & 9 & 2 & 7 & 8.5 & 18173.7005 & -0.0011 \\
\hline 10 & 2 & 8 & 8.5 & 9 & 2 & 7 & 7.5 & 18173.8393 & -0.0024 \\
\hline 10 & 2 & 8 & 11.5 & 9 & 2 & 7 & 10.5 & 18174.4971 & -0.0012 \\
\hline 11 & 1 & 10 & 11.5 & 10 & 1 & 9 & 10.5 & 18213.6433 & -0.0012 \\
\hline 11 & 1 & 10 & 10.5 & 10 & 1 & 9 & 9.5 & 18214.0346 & -0.0006 \\
\hline 11 & 1 & 10 & 12.5 & 10 & 1 & 9 & 11.5 & 18216.1473 & -0.0004 \\
\hline 11 & 1 & 10 & 9.5 & 10 & 1 & 9 & 8.5 & 18216.5327 & -0.0004 \\
\hline 6 & 3 & 4 & 6.5 & 5 & 2 & 3 & 5.5 & 18762.0763 & -0.0004 \\
\hline 6 & 3 & 4 & 5.5 & 5 & 2 & 3 & 4.5 & 18765.7670 & -0.0009 \\
\hline 6 & 3 & 4 & 7.5 & 5 & 2 & 3 & 6.5 & 18772.8990 & 0.0005 \\
\hline 6 & 3 & 4 & 4.5 & 5 & 2 & 3 & 3.5 & 18775.5502 & -0.0022 \\
\hline 6 & 2 & 4 & 4.5 & 5 & 1 & 5 & 3.5 & 19340.7191 & 0.0012 \\
\hline 6 & 2 & 4 & 7.5 & 5 & 1 & 5 & 6.5 & 19347.3668 & 0.0010 \\
\hline 6 & 2 & 4 & 5.5 & 5 & 1 & 5 & 4.5 & 19367.1992 & 0.0014 \\
\hline 6 & 2 & 4 & 6.5 & 5 & 1 & 5 & 5.5 & 19373.8249 & 0.0022 \\
\hline 4 & 4 & 1 & 3.5 & 3 & 3 & 0 & 2.5 & 19552.2533 & -0.0007 \\
\hline 4 & 4 & 1 & 4.5 & 3 & 3 & 0 & 3.5 & 19552.9333 & -0.0001 \\
\hline
\end{tabular}


Table S2: Observed Transition Frequencies (in $\mathrm{MHz}$ ) for $\mathrm{CF}_{2} \mathrm{CH}^{35} \mathrm{Cl}-\mathrm{HCCH}$

\begin{tabular}{|r|r|c|c|c|c|c|c|r|r|}
\hline$J^{\prime}$ & $K_{a}{ }^{\prime}$ & $K_{c}{ }^{\prime}$ & $F^{\prime}$ & $J^{\prime \prime}$ & $K_{a}{ }^{\prime \prime}$ & $K_{c}{ }^{\prime \prime}$ & $F^{\prime \prime}$ & Observed & Obs - Calc \\
\hline 4 & 4 & 1 & 2.5 & 3 & 3 & 0 & 1.5 & 19553.8320 & 0.0008 \\
\hline 4 & 4 & 0 & 3.5 & 3 & 3 & 1 & 2.5 & 19554.4125 & 0.0004 \\
\hline 4 & 4 & 1 & 5.5 & 3 & 3 & 0 & 4.5 & 19554.5198 & 0.0030 \\
\hline 4 & 4 & 0 & 4.5 & 3 & 3 & 1 & 3.5 & 19555.1441 & 0.0001 \\
\hline 4 & 4 & 0 & 2.5 & 3 & 3 & 1 & 1.5 & 19555.8787 & 0.0010 \\
\hline 4 & 4 & 0 & 5.5 & 3 & 3 & 1 & 4.5 & 19556.6150 & 0.0018 \\
\hline 11 & 2 & 9 & 11.5 & 10 & 2 & 8 & 10.5 & 19810.1536 & -0.0056 \\
\hline 11 & 2 & 9 & 9.5 & 10 & 2 & 8 & 8.5 & 19811.2827 & -0.0001 \\
\hline 6 & 3 & 3 & 4.5 & 5 & 2 & 4 & 3.5 & 19847.2479 & 0.0012 \\
\hline 6 & 3 & 3 & 7.5 & 5 & 2 & 4 & 6.5 & 19847.9925 & 0.0003 \\
\hline 6 & 3 & 3 & 5.5 & 5 & 2 & 4 & 4.5 & 19851.0403 & -0.0010 \\
\hline 6 & 3 & 3 & 6.5 & 5 & 2 & 4 & 5.5 & 19851.6537 & 0.0008 \\
\hline 7 & 3 & 5 & 6.5 & 6 & 2 & 4 & 5.5 & 19966.9046 & -0.0012 \\
\hline 7 & 3 & 5 & 8.5 & 6 & 2 & 4 & 7.5 & 19975.5317 & 0.0000 \\
\hline 7 & 3 & 5 & 5.5 & 6 & 2 & 4 & 4.5 & 19978.5157 & -0.0011 \\
\hline
\end{tabular}


Table S3: Observed Transition Frequencies (in $\mathrm{MHz}$ ) for $\mathrm{CF}_{2} \mathrm{CH}^{37} \mathrm{Cl}-\mathrm{HCCH}$

\begin{tabular}{|c|c|c|c|c|c|c|c|c|c|}
\hline$J^{\prime}$ & $K_{a}^{\prime}$ & $K_{c}{ }^{\prime}$ & $F^{\prime}$ & $J^{\prime \prime}$ & $K_{a}^{\prime \prime}$ & $K_{c}^{\prime \prime}$ & $F^{\prime \prime}$ & Observed & Obs - Calc \\
\hline 4 & 0 & 4 & 5.5 & 3 & 1 & 3 & 4.5 & 5452.8714 & -0.0008 \\
\hline 4 & 0 & 4 & 4.5 & 3 & 1 & 3 & 3.5 & 5458.8543 & -0.0010 \\
\hline 3 & 1 & 2 & 4.5 & 2 & 1 & 1 & 3.5 & 5497.7825 & 0.0009 \\
\hline 3 & 1 & 2 & 3.5 & 2 & 1 & 1 & 2.5 & 5498.9796 & 0.0001 \\
\hline 3 & 1 & 2 & 1.5 & 2 & 1 & 1 & 0.5 & 5500.7711 & -0.0004 \\
\hline 3 & 1 & 2 & 2.5 & 2 & 1 & 1 & 1.5 & 5501.9638 & -0.0008 \\
\hline 3 & 1 & 3 & 3.5 & 2 & 0 & 2 & 2.5 & 6047.2040 & -0.0002 \\
\hline 3 & 1 & 3 & 2.5 & 2 & 0 & 2 & 2.5 & 6049.9548 & 0.0013 \\
\hline 3 & 1 & 3 & 2.5 & 2 & 0 & 2 & 1.5 & 6053.7899 & 0.0009 \\
\hline 3 & 1 & 3 & 4.5 & 2 & 0 & 2 & 3.5 & 6058.4646 & -0.0008 \\
\hline 3 & 1 & 3 & 1.5 & 2 & 0 & 2 & 0.5 & 6065.1034 & -0.0003 \\
\hline 4 & 1 & 4 & 4.5 & 3 & 1 & 3 & 4.5 & 6208.8966 & -0.0012 \\
\hline 4 & 1 & 4 & 3.5 & 3 & 1 & 3 & 2.5 & 6214.0222 & -0.0018 \\
\hline 4 & 1 & 4 & 2.5 & 3 & 1 & 3 & 1.5 & 6214.0444 & 0.0027 \\
\hline 4 & 1 & 4 & 5.5 & 3 & 1 & 3 & 4.5 & 6214.6806 & 0.0004 \\
\hline 4 & 1 & 4 & 4.5 & 3 & 1 & 3 & 3.5 & 6214.7500 & -0.0003 \\
\hline 4 & 1 & 4 & 3.5 & 3 & 1 & 3 & 3.5 & 6216.7724 & -0.0010 \\
\hline 4 & 1 & 4 & 2.5 & 3 & 1 & 3 & 2.5 & 6219.9243 & 0.0007 \\
\hline 4 & 0 & 4 & 4.5 & 3 & 0 & 3 & 3.5 & 6520.0320 & 0.0011 \\
\hline 4 & 0 & 4 & 2.5 & 3 & 0 & 3 & 2.5 & 6521.1091 & 0.0002 \\
\hline 4 & 0 & 4 & 3.5 & 3 & 0 & 3 & 2.5 & 6521.3913 & -0.0004 \\
\hline 4 & 0 & 4 & 5.5 & 3 & 0 & 3 & 4.5 & 6522.8674 & 0.0000 \\
\hline 4 & 0 & 4 & 4.5 & 3 & 0 & 3 & 4.5 & 6522.9975 & -0.0007 \\
\hline 4 & 0 & 4 & 2.5 & 3 & 0 & 3 & 1.5 & 6524.1114 & -0.0002 \\
\hline 4 & 2 & 3 & 2.5 & 4 & 1 & 4 & 2.5 & 6645.1602 & -0.0020 \\
\hline 4 & 2 & 3 & 5.5 & 4 & 1 & 4 & 5.5 & 6648.0522 & 0.0017 \\
\hline 4 & 2 & 3 & 3.5 & 4 & 1 & 4 & 3.5 & 6653.3633 & -0.0012 \\
\hline 4 & 2 & 3 & 4.5 & 4 & 1 & 4 & 4.5 & 6656.3641 & -0.0019 \\
\hline 4 & 2 & 3 & 2.5 & 3 & 2 & 2 & 1.5 & 6789.1577 & 0.0009 \\
\hline 4 & 2 & 3 & 5.5 & 3 & 2 & 2 & 4.5 & 6789.9131 & -0.0002 \\
\hline 4 & 2 & 3 & 3.5 & 3 & 2 & 2 & 2.5 & 6791.4375 & -0.0005 \\
\hline 4 & 2 & 3 & 4.5 & 3 & 2 & 2 & 3.5 & 6792.4469 & -0.0001 \\
\hline 4 & 3 & 2 & 2.5 & 3 & 3 & 1 & 1.5 & 6866.7362 & 0.0008 \\
\hline 4 & 3 & 2 & 5.5 & 3 & 3 & 1 & 4.5 & 6870.2080 & -0.0001 \\
\hline 4 & 3 & 2 & 3.5 & 3 & 3 & 1 & 2.5 & 6873.8453 & 0.0007 \\
\hline 4 & 3 & 2 & 4.5 & 3 & 3 & 1 & 3.5 & 6877.2924 & -0.0001 \\
\hline 4 & 3 & 1 & 2.5 & 3 & 3 & 0 & 1.5 & 6880.3171 & -0.0009 \\
\hline 4 & 3 & 1 & 5.5 & 3 & 3 & 0 & 4.5 & 6883.8614 & -0.0011 \\
\hline 4 & 3 & 1 & 3.5 & 3 & 3 & 0 & 2.5 & 6887.6923 & 0.0000 \\
\hline 4 & 3 & 1 & 4.5 & 3 & 3 & 0 & 3.5 & 6891.2697 & 0.0002 \\
\hline 4 & 2 & 2 & 2.5 & 3 & 2 & 1 & 1.5 & 7081.0314 & 0.0025 \\
\hline 4 & 2 & 2 & 5.5 & 3 & 2 & 1 & 4.5 & 7082.3981 & -0.0003 \\
\hline 4 & 2 & 2 & 3.5 & 3 & 2 & 1 & 2.5 & 7086.2721 & -0.0002 \\
\hline
\end{tabular}


Table S3: Observed Transition Frequencies (in $\mathrm{MHz}$ ) for $\mathrm{CF}_{2} \mathrm{CH}^{37} \mathrm{Cl}-\mathrm{HCCH}$

\begin{tabular}{|c|c|c|c|c|c|c|c|c|c|}
\hline$J^{\prime}$ & $K_{a}^{\prime}$ & $K_{c}{ }^{\prime}$ & $F^{\prime}$ & $J^{\prime \prime}$ & $K_{a}^{\prime \prime}$ & $K_{c}^{\prime \prime}$ & $F^{\prime \prime}$ & Observed & Obs - Calc \\
\hline 4 & 2 & 2 & 4.5 & 3 & 2 & 1 & 3.5 & 7087.6187 & 0.0013 \\
\hline 4 & 2 & 2 & 4.5 & 3 & 2 & 1 & 4.5 & 7091.7869 & -0.0008 \\
\hline 5 & 0 & 5 & 3.5 & 4 & 1 & 4 & 2.5 & 7222.6259 & 0.0008 \\
\hline 5 & 0 & 5 & 6.5 & 4 & 1 & 4 & 5.5 & 7223.9358 & 0.0006 \\
\hline 5 & 0 & 5 & 4.5 & 4 & 1 & 4 & 3.5 & 7225.9551 & -0.0003 \\
\hline 5 & 0 & 5 & 5.5 & 4 & 1 & 4 & 4.5 & 7227.3450 & 0.0017 \\
\hline 4 & 1 & 3 & 2.5 & 3 & 1 & 2 & 2.5 & 7275.1347 & 0.0008 \\
\hline 4 & 1 & 4 & 4.5 & 3 & 0 & 3 & 3.5 & 7275.9252 & -0.0006 \\
\hline 4 & 1 & 4 & 3.5 & 3 & 0 & 3 & 3.5 & 7277.9450 & -0.0040 \\
\hline 4 & 1 & 4 & 4.5 & 3 & 0 & 3 & 4.5 & 7278.8926 & -0.0005 \\
\hline 4 & 1 & 4 & 3.5 & 3 & 0 & 3 & 2.5 & 7279.3170 & 0.0009 \\
\hline 4 & 1 & 3 & 3.5 & 3 & 1 & 2 & 3.5 & 7283.0733 & 0.0005 \\
\hline 4 & 1 & 4 & 5.5 & 3 & 0 & 3 & 4.5 & 7284.6753 & -0.0002 \\
\hline 4 & 1 & 4 & 2.5 & 3 & 0 & 3 & 1.5 & 7288.2175 & -0.0009 \\
\hline 4 & 1 & 3 & 4.5 & 3 & 1 & 2 & 3.5 & 7288.4187 & -0.0003 \\
\hline 4 & 1 & 3 & 5.5 & 3 & 1 & 2 & 4.5 & 7288.6936 & -0.0008 \\
\hline 4 & 1 & 3 & 3.5 & 3 & 1 & 2 & 2.5 & 7290.3140 & -0.0003 \\
\hline 4 & 1 & 3 & 2.5 & 3 & 1 & 2 & 1.5 & 7290.5710 & 0.0010 \\
\hline 4 & 1 & 3 & 4.5 & 3 & 1 & 2 & 4.5 & 7303.9100 & 0.0002 \\
\hline 5 & 2 & 4 & 3.5 & 5 & 1 & 5 & 3.5 & 7371.1407 & -0.0023 \\
\hline 5 & 1 & 5 & 5.5 & 4 & 1 & 4 & 5.5 & 7716.8462 & -0.0001 \\
\hline 5 & 1 & 5 & 4.5 & 4 & 1 & 4 & 3.5 & 7722.3648 & -0.0005 \\
\hline 5 & 1 & 5 & 5.5 & 4 & 1 & 4 & 4.5 & 7722.6290 & 0.0003 \\
\hline 5 & 1 & 5 & 3.5 & 4 & 1 & 4 & 2.5 & 7722.7249 & -0.0014 \\
\hline 5 & 1 & 5 & 6.5 & 4 & 1 & 4 & 5.5 & 7723.0708 & -0.0002 \\
\hline 5 & 1 & 5 & 4.5 & 4 & 1 & 4 & 4.5 & 7724.3873 & -0.0011 \\
\hline 5 & 1 & 5 & 3.5 & 4 & 1 & 4 & 3.5 & 7728.6261 & 0.0002 \\
\hline 5 & 0 & 5 & 5.5 & 4 & 0 & 4 & 4.5 & 7983.2377 & -0.0006 \\
\hline 5 & 0 & 5 & 4.5 & 4 & 0 & 4 & 3.5 & 7983.8796 & -0.0002 \\
\hline 5 & 0 & 5 & 6.5 & 4 & 0 & 4 & 5.5 & 7985.7436 & 0.0003 \\
\hline 5 & 0 & 5 & 3.5 & 4 & 0 & 4 & 3.5 & 7986.4501 & 0.0010 \\
\hline 5 & 0 & 5 & 3.5 & 4 & 0 & 4 & 2.5 & 7986.7320 & 0.0000 \\
\hline 5 & 2 & 4 & 3.5 & 4 & 2 & 3 & 2.5 & 8448.7072 & 0.0001 \\
\hline 5 & 2 & 4 & 6.5 & 4 & 2 & 3 & 5.5 & 8448.8387 & 0.0002 \\
\hline 5 & 2 & 4 & 5.5 & 4 & 2 & 3 & 4.5 & 8449.5781 & -0.0018 \\
\hline 5 & 2 & 4 & 4.5 & 4 & 2 & 3 & 3.5 & 8449.6070 & 0.0026 \\
\hline 5 & 1 & 5 & 5.5 & 4 & 0 & 4 & 4.5 & 8478.5238 & 0.0002 \\
\hline 5 & 1 & 5 & 4.5 & 4 & 0 & 4 & 3.5 & 8480.2898 & 0.0001 \\
\hline 5 & 1 & 5 & 6.5 & 4 & 0 & 4 & 5.5 & 8484.8794 & 0.0004 \\
\hline 5 & 1 & 5 & 3.5 & 4 & 0 & 4 & 2.5 & 8486.8323 & -0.0007 \\
\hline 2 & 2 & 1 & 2.5 & 1 & 1 & 0 & 1.5 & 8500.7448 & 0.0009 \\
\hline 2 & 2 & 1 & 1.5 & 1 & 1 & 0 & 1.5 & 8505.8543 & -0.0010 \\
\hline 2 & 2 & 1 & 2.5 & 1 & 1 & 0 & 2.5 & 8507.8803 & -0.0015 \\
\hline
\end{tabular}


Table S3: Observed Transition Frequencies (in $\mathrm{MHz}$ ) for $\mathrm{CF}_{2} \mathrm{CH}^{37} \mathrm{Cl}-\mathrm{HCCH}$

\begin{tabular}{|c|c|c|c|c|c|c|c|c|c|}
\hline$J^{\prime}$ & $K_{a}^{\prime}$ & $K_{c}^{\prime}$ & $F^{\prime}$ & $J^{\prime \prime}$ & $K_{a}^{\prime \prime}$ & $K_{c}^{\prime \prime}$ & $F^{\prime \prime}$ & Observed & Obs - Calc \\
\hline 2 & 2 & 1 & 3.5 & 1 & 1 & 0 & 2.5 & 8515.0019 & 0.0007 \\
\hline 2 & 2 & 1 & 1.5 & 1 & 1 & 0 & 0.5 & 8518.7446 & -0.0002 \\
\hline 2 & 2 & 1 & 0.5 & 1 & 1 & 0 & 0.5 & 8525.8579 & 0.0006 \\
\hline 5 & 3 & 3 & 3.5 & 4 & 3 & 2 & 2.5 & 8603.4277 & -0.0008 \\
\hline 5 & 3 & 3 & 6.5 & 4 & 3 & 2 & 5.5 & 8604.5441 & -0.0005 \\
\hline 5 & 3 & 3 & 4.5 & 4 & 3 & 2 & 3.5 & 8607.0637 & 0.0007 \\
\hline 5 & 3 & 3 & 5.5 & 4 & 3 & 2 & 4.5 & 8608.1905 & 0.0006 \\
\hline 5 & 3 & 2 & 3.5 & 4 & 3 & 1 & 2.5 & 8650.3561 & -0.0017 \\
\hline 5 & 3 & 2 & 6.5 & 4 & 3 & 1 & 5.5 & 8651.6394 & -0.0010 \\
\hline 5 & 3 & 2 & 4.5 & 4 & 3 & 1 & 3.5 & 8654.6506 & -0.0005 \\
\hline 5 & 3 & 2 & 5.5 & 4 & 3 & 1 & 4.5 & 8655.9091 & -0.0005 \\
\hline 2 & 2 & 0 & 1.5 & 1 & 1 & 1 & 0.5 & 8800.9563 & 0.0016 \\
\hline 2 & 2 & 0 & 0.5 & 1 & 1 & 1 & 0.5 & 8806.4058 & 0.0006 \\
\hline 2 & 2 & 0 & 2.5 & 1 & 1 & 1 & 2.5 & 8808.5584 & -0.0039 \\
\hline 6 & 0 & 6 & 7.5 & 5 & 1 & 5 & 6.5 & 8905.5513 & 0.0008 \\
\hline 6 & 0 & 6 & 5.5 & 5 & 1 & 5 & 4.5 & 8906.4952 & -0.0012 \\
\hline 6 & 0 & 6 & 6.5 & 5 & 1 & 5 & 5.5 & 8907.1820 & 0.0001 \\
\hline 5 & 2 & 3 & 3.5 & 4 & 2 & 2 & 2.5 & 8980.8046 & -0.0002 \\
\hline 5 & 2 & 3 & 6.5 & 4 & 2 & 2 & 5.5 & 8981.1200 & -0.0004 \\
\hline 5 & 2 & 3 & 4.5 & 4 & 2 & 2 & 3.5 & 8984.2257 & 0.0005 \\
\hline 5 & 2 & 3 & 5.5 & 4 & 2 & 2 & 4.5 & 8984.5529 & -0.0007 \\
\hline 5 & 1 & 4 & 4.5 & 4 & 1 & 3 & 4.5 & 9030.8062 & 0.0002 \\
\hline 5 & 1 & 4 & 5.5 & 4 & 1 & 3 & 4.5 & 9034.8099 & -0.0001 \\
\hline 5 & 1 & 4 & 6.5 & 4 & 1 & 3 & 5.5 & 9035.8554 & -0.0003 \\
\hline 5 & 1 & 4 & 4.5 & 4 & 1 & 3 & 3.5 & 9036.1525 & 0.0004 \\
\hline 5 & 1 & 4 & 3.5 & 4 & 1 & 3 & 2.5 & 9037.1848 & -0.0005 \\
\hline 5 & 1 & 4 & 5.5 & 4 & 1 & 3 & 5.5 & 9050.0239 & -0.0015 \\
\hline 6 & 1 & 6 & 6.5 & 5 & 1 & 5 & 6.5 & 9204.0741 & 0.0012 \\
\hline 6 & 1 & 6 & 5.5 & 5 & 1 & 5 & 4.5 & 9210.1510 & 0.0003 \\
\hline 6 & 1 & 6 & 6.5 & 5 & 1 & 5 & 5.5 & 9210.2969 & -0.0007 \\
\hline 6 & 1 & 6 & 4.5 & 5 & 1 & 5 & 3.5 & 9210.7181 & -0.0005 \\
\hline 6 & 1 & 6 & 7.5 & 5 & 1 & 5 & 6.5 & 9210.8744 & -0.0001 \\
\hline 6 & 1 & 6 & 5.5 & 5 & 1 & 5 & 5.5 & 9211.9095 & -0.0009 \\
\hline 6 & 1 & 6 & 4.5 & 5 & 1 & 5 & 4.5 & 9216.9782 & -0.0010 \\
\hline 6 & 0 & 6 & 6.5 & 5 & 0 & 5 & 6.5 & 9400.0898 & -0.0032 \\
\hline 6 & 0 & 6 & 6.5 & 5 & 0 & 5 & 5.5 & 9402.4673 & -0.0001 \\
\hline 6 & 0 & 6 & 5.5 & 5 & 0 & 5 & 4.5 & 9402.9063 & 0.0000 \\
\hline 6 & 0 & 6 & 5.5 & 5 & 0 & 5 & 5.5 & 9403.5430 & 0.0015 \\
\hline 6 & 0 & 6 & 7.5 & 5 & 0 & 5 & 6.5 & 9404.6868 & 0.0005 \\
\hline 6 & 0 & 6 & 4.5 & 5 & 0 & 5 & 3.5 & 9405.0102 & -0.0004 \\
\hline 6 & 0 & 6 & 4.5 & 5 & 0 & 5 & 4.5 & 9407.5778 & -0.0022 \\
\hline 6 & 1 & 6 & 6.5 & 5 & 0 & 5 & 5.5 & 9705.5824 & -0.0007 \\
\hline 6 & 1 & 6 & 5.5 & 5 & 0 & 5 & 4.5 & 9706.5606 & 0.0000 \\
\hline
\end{tabular}


Table S3: Observed Transition Frequencies (in $\mathrm{MHz}$ ) for $\mathrm{CF}_{2} \mathrm{CH}^{37} \mathrm{Cl}-\mathrm{HCCH}$

\begin{tabular}{|c|c|c|c|c|c|c|c|c|c|}
\hline$J^{\prime}$ & $K_{a}^{\prime}$ & $K_{c}{ }^{\prime}$ & $F^{\prime}$ & $J^{\prime \prime}$ & $K_{a}^{\prime \prime}$ & $K_{c}^{\prime \prime}$ & $F^{\prime \prime}$ & Observed & Obs - Calc \\
\hline 6 & 1 & 6 & 7.5 & 5 & 0 & 5 & 6.5 & 9710.0104 & 0.0000 \\
\hline 6 & 1 & 6 & 4.5 & 5 & 0 & 5 & 3.5 & 9710.8197 & 0.0000 \\
\hline 3 & 2 & 2 & 3.5 & 2 & 1 & 1 & 2.5 & 9933.1012 & -0.0002 \\
\hline 3 & 2 & 2 & 2.5 & 2 & 1 & 1 & 1.5 & 9943.3578 & -0.0007 \\
\hline 3 & 2 & 2 & 4.5 & 2 & 1 & 1 & 3.5 & 9947.3953 & 0.0005 \\
\hline 3 & 2 & 2 & 1.5 & 2 & 1 & 1 & 0.5 & 9957.5797 & -0.0002 \\
\hline 6 & 2 & 5 & 7.5 & 5 & 2 & 4 & 6.5 & 10081.9714 & -0.0012 \\
\hline 6 & 2 & 5 & 6.5 & 5 & 2 & 4 & 5.5 & 10082.0052 & -0.0004 \\
\hline 6 & 2 & 5 & 4.5 & 5 & 2 & 4 & 3.5 & 10082.1275 & 0.0001 \\
\hline 6 & 2 & 5 & 5.5 & 5 & 2 & 4 & 4.5 & 10082.1482 & 0.0003 \\
\hline 6 & 4 & 3 & 4.5 & 5 & 4 & 2 & 3.5 & 10326.5211 & 0.0005 \\
\hline 6 & 4 & 3 & 7.5 & 5 & 4 & 2 & 6.5 & 10327.6370 & -0.0004 \\
\hline 6 & 4 & 3 & 5.5 & 5 & 4 & 2 & 4.5 & 10330.3828 & -0.0008 \\
\hline 6 & 4 & 3 & 6.5 & 5 & 4 & 2 & 5.5 & 10331.3414 & -0.0001 \\
\hline 6 & 4 & 2 & 4.5 & 5 & 4 & 1 & 3.5 & 10331.4597 & -0.0011 \\
\hline 6 & 4 & 2 & 7.5 & 5 & 4 & 1 & 6.5 & 10332.5012 & -0.0012 \\
\hline 6 & 4 & 2 & 5.5 & 5 & 4 & 1 & 4.5 & 10335.2825 & -0.0012 \\
\hline 6 & 4 & 2 & 6.5 & 5 & 4 & 1 & 5.5 & 10336.3608 & 0.0002 \\
\hline 6 & 3 & 4 & 4.5 & 5 & 3 & 3 & 3.5 & 10338.5613 & 0.0004 \\
\hline 6 & 3 & 4 & 7.5 & 5 & 3 & 3 & 6.5 & 10338.8504 & -0.0005 \\
\hline 6 & 3 & 4 & 5.5 & 5 & 3 & 3 & 4.5 & 10340.5064 & -0.0001 \\
\hline 6 & 3 & 4 & 6.5 & 5 & 3 & 3 & 5.5 & 10341.0225 & 0.0000 \\
\hline 6 & 3 & 3 & 4.5 & 5 & 3 & 2 & 3.5 & 10459.5864 & 0.0011 \\
\hline 6 & 3 & 3 & 7.5 & 5 & 3 & 2 & 6.5 & 10460.1858 & -0.0006 \\
\hline 6 & 3 & 3 & 5.5 & 5 & 3 & 2 & 4.5 & 10462.7517 & 0.0004 \\
\hline 6 & 3 & 3 & 6.5 & 5 & 3 & 2 & 5.5 & 10463.3399 & 0.0000 \\
\hline 7 & 0 & 7 & 5.5 & 6 & 1 & 6 & 4.5 & 10503.4501 & -0.0002 \\
\hline 7 & 0 & 7 & 8.5 & 6 & 1 & 6 & 7.5 & 10503.7746 & 0.0001 \\
\hline 7 & 0 & 7 & 6.5 & 6 & 1 & 6 & 5.5 & 10504.0023 & -0.0001 \\
\hline 7 & 0 & 7 & 7.5 & 6 & 1 & 6 & 6.5 & 10504.3553 & -0.0007 \\
\hline 7 & 1 & 7 & 7.5 & 6 & 1 & 6 & 7.5 & 10673.6126 & -0.0011 \\
\hline 7 & 1 & 7 & 6.5 & 6 & 1 & 6 & 5.5 & 10680.3166 & -0.0004 \\
\hline 7 & 1 & 7 & 7.5 & 6 & 1 & 6 & 6.5 & 10680.4152 & -0.0002 \\
\hline 7 & 1 & 7 & 5.5 & 6 & 1 & 6 & 4.5 & 10680.9322 & 0.0001 \\
\hline 7 & 1 & 7 & 8.5 & 6 & 1 & 6 & 7.5 & 10681.0334 & 0.0000 \\
\hline 7 & 1 & 7 & 6.5 & 6 & 1 & 6 & 6.5 & 10681.9311 & 0.0013 \\
\hline 7 & 1 & 7 & 5.5 & 6 & 1 & 6 & 5.5 & 10687.7629 & 0.0024 \\
\hline 6 & 1 & 5 & 4.5 & 5 & 1 & 4 & 4.5 & 10708.3508 & 0.0013 \\
\hline 6 & 1 & 5 & 5.5 & 5 & 1 & 4 & 5.5 & 10716.8561 & -0.0005 \\
\hline 6 & 1 & 5 & 6.5 & 5 & 1 & 4 & 5.5 & 10719.8109 & -0.0009 \\
\hline 6 & 1 & 5 & 5.5 & 5 & 1 & 4 & 4.5 & 10720.8599 & -0.0007 \\
\hline 6 & 1 & 5 & 7.5 & 5 & 1 & 4 & 6.5 & 10721.4749 & -0.0002 \\
\hline 6 & 1 & 5 & 4.5 & 5 & 1 & 4 & 3.5 & 10722.4970 & 0.0003 \\
\hline
\end{tabular}


Table S3: Observed Transition Frequencies (in $\mathrm{MHz}$ ) for $\mathrm{CF}_{2} \mathrm{CH}^{37} \mathrm{Cl}-\mathrm{HCCH}$

\begin{tabular}{|c|c|c|c|c|c|c|c|c|c|}
\hline$J^{\prime}$ & $K_{a}^{\prime}$ & $K_{c}^{\prime}$ & $F^{\prime}$ & $J^{\prime \prime}$ & $K_{a}^{\prime \prime}$ & $K_{c}^{\prime \prime}$ & $F^{\prime \prime}$ & Observed & Obs - Calc \\
\hline 6 & 1 & 5 & 6.5 & 5 & 1 & 4 & 6.5 & 10733.9816 & 0.0001 \\
\hline 7 & 0 & 7 & 7.5 & 6 & 0 & 6 & 7.5 & 10802.8789 & 0.0006 \\
\hline 7 & 0 & 7 & 7.5 & 6 & 0 & 6 & 6.5 & 10807.4707 & -0.0009 \\
\hline 7 & 0 & 7 & 6.5 & 6 & 0 & 6 & 5.5 & 10807.6556 & -0.0012 \\
\hline 7 & 0 & 7 & 6.5 & 6 & 0 & 6 & 6.5 & 10808.7309 & 0.0000 \\
\hline 7 & 0 & 7 & 8.5 & 6 & 0 & 6 & 7.5 & 10809.0987 & 0.0003 \\
\hline 7 & 0 & 7 & 5.5 & 6 & 0 & 6 & 4.5 & 10809.2588 & -0.0005 \\
\hline 7 & 0 & 7 & 5.5 & 6 & 0 & 6 & 5.5 & 10813.9332 & 0.0001 \\
\hline 6 & 2 & 4 & 7.5 & 5 & 2 & 3 & 6.5 & 10895.1862 & -0.0002 \\
\hline 6 & 2 & 4 & 4.5 & 5 & 2 & 3 & 3.5 & 10895.2540 & 0.0000 \\
\hline 6 & 2 & 4 & 6.5 & 5 & 2 & 3 & 5.5 & 10897.3008 & -0.0007 \\
\hline 6 & 2 & 4 & 5.5 & 5 & 2 & 3 & 4.5 & 10897.3892 & -0.0021 \\
\hline 3 & 2 & 1 & 1.5 & 2 & 1 & 2 & 0.5 & 10904.6153 & 0.0015 \\
\hline 3 & 2 & 1 & 4.5 & 2 & 1 & 2 & 3.5 & 10911.6928 & 0.0014 \\
\hline 3 & 2 & 1 & 2.5 & 2 & 1 & 2 & 1.5 & 10915.8962 & 0.0006 \\
\hline 3 & 2 & 1 & 3.5 & 2 & 1 & 2 & 2.5 & 10922.9853 & 0.0004 \\
\hline 7 & 1 & 7 & 7.5 & 6 & 0 & 6 & 6.5 & 10983.5310 & 0.0000 \\
\hline 7 & 1 & 7 & 6.5 & 6 & 0 & 6 & 5.5 & 10983.9714 & 0.0001 \\
\hline 7 & 1 & 7 & 8.5 & 6 & 0 & 6 & 7.5 & 10986.3573 & -0.0001 \\
\hline 7 & 1 & 7 & 5.5 & 6 & 0 & 6 & 4.5 & 10986.7413 & 0.0001 \\
\hline 4 & 2 & 3 & 4.5 & 3 & 1 & 2 & 3.5 & 11226.5699 & 0.0010 \\
\hline 4 & 2 & 3 & 3.5 & 3 & 1 & 2 & 2.5 & 11232.8318 & -0.0002 \\
\hline 4 & 2 & 3 & 5.5 & 3 & 1 & 2 & 4.5 & 11239.5271 & 0.0006 \\
\hline 4 & 2 & 3 & 2.5 & 3 & 1 & 2 & 1.5 & 11245.9652 & 0.0000 \\
\hline 7 & 2 & 6 & 7.5 & 6 & 2 & 5 & 6.5 & 11685.8690 & 0.0000 \\
\hline 7 & 2 & 6 & 6.5 & 6 & 2 & 5 & 5.5 & 11686.0698 & -0.0007 \\
\hline 7 & 2 & 6 & 8.5 & 6 & 2 & 5 & 7.5 & 11686.2809 & -0.0001 \\
\hline 7 & 2 & 6 & 5.5 & 6 & 2 & 5 & 4.5 & 11686.4594 & -0.0004 \\
\hline 8 & 0 & 8 & 6.5 & 7 & 1 & 7 & 5.5 & 12038.2869 & -0.0032 \\
\hline 8 & 0 & 8 & 7.5 & 7 & 1 & 7 & 6.5 & 12038.2997 & 0.0024 \\
\hline 8 & 0 & 8 & 9.5 & 7 & 1 & 7 & 8.5 & 12038.4669 & 0.0007 \\
\hline 8 & 0 & 8 & 8.5 & 7 & 1 & 7 & 7.5 & 12038.4914 & -0.0032 \\
\hline 7 & 3 & 5 & 5.5 & 6 & 3 & 4 & 4.5 & 12066.5984 & 0.0001 \\
\hline 7 & 3 & 5 & 8.5 & 6 & 3 & 4 & 7.5 & 12066.8619 & 0.0014 \\
\hline 7 & 3 & 5 & 7.5 & 6 & 3 & 4 & 6.5 & 12067.9284 & 0.0003 \\
\hline 7 & 3 & 5 & 6.5 & 6 & 3 & 4 & 5.5 & 12067.9915 & 0.0013 \\
\hline 8 & 1 & 8 & 7.5 & 7 & 1 & 7 & 6.5 & 12136.8171 & 0.0009 \\
\hline 8 & 1 & 8 & 8.5 & 7 & 1 & 7 & 7.5 & 12136.8934 & -0.0004 \\
\hline 8 & 1 & 8 & 6.5 & 7 & 1 & 7 & 5.5 & 12137.4125 & -0.0002 \\
\hline 8 & 1 & 8 & 9.5 & 7 & 1 & 7 & 8.5 & 12137.4911 & 0.0003 \\
\hline 8 & 0 & 8 & 8.5 & 7 & 0 & 7 & 7.5 & 12214.5549 & 0.0008 \\
\hline 8 & 0 & 8 & 7.5 & 7 & 0 & 7 & 6.5 & 12214.6131 & 0.0012 \\
\hline 8 & 0 & 8 & 9.5 & 7 & 0 & 7 & 8.5 & 12215.7269 & 0.0017 \\
\hline
\end{tabular}


Table S3: Observed Transition Frequencies (in $\mathrm{MHz}$ ) for $\mathrm{CF}_{2} \mathrm{CH}^{37} \mathrm{Cl}-\mathrm{HCCH}$

\begin{tabular}{|c|c|c|c|c|c|c|c|c|c|}
\hline$J^{\prime}$ & $K_{a}^{\prime}$ & $K_{c}{ }^{\prime}$ & $F^{\prime}$ & $J^{\prime \prime}$ & $K_{a}^{\prime \prime}$ & $K_{c}^{\prime \prime}$ & $F^{\prime \prime}$ & Observed & Obs - Calc \\
\hline 8 & 0 & 8 & 6.5 & 7 & 0 & 7 & 5.5 & 12215.7720 & 0.0000 \\
\hline 8 & 1 & 8 & 8.5 & 7 & 0 & 7 & 7.5 & 12312.9531 & -0.0002 \\
\hline 8 & 1 & 8 & 6.5 & 7 & 0 & 7 & 5.5 & 12314.8946 & 0.0002 \\
\hline 7 & 1 & 6 & 5.5 & 6 & 1 & 5 & 5.5 & 12316.5730 & 0.0006 \\
\hline 7 & 3 & 4 & 5.5 & 6 & 3 & 3 & 4.5 & 12322.8138 & 0.0007 \\
\hline 7 & 3 & 4 & 8.5 & 6 & 3 & 3 & 7.5 & 12323.1392 & 0.0004 \\
\hline 7 & 1 & 6 & 6.5 & 6 & 1 & 5 & 6.5 & 12323.8300 & 0.0022 \\
\hline 7 & 3 & 4 & 6.5 & 6 & 3 & 3 & 5.5 & 12325.5426 & 0.0007 \\
\hline 7 & 3 & 4 & 7.5 & 6 & 3 & 3 & 6.5 & 12325.9040 & 0.0008 \\
\hline 7 & 1 & 6 & 7.5 & 6 & 1 & 5 & 6.5 & 12326.0291 & 0.0001 \\
\hline 7 & 1 & 6 & 8.5 & 6 & 1 & 5 & 7.5 & 12328.0887 & 0.0008 \\
\hline 7 & 1 & 6 & 5.5 & 6 & 1 & 5 & 4.5 & 12329.0837 & 0.0002 \\
\hline 5 & 2 & 4 & 5.5 & 4 & 1 & 3 & 4.5 & 12387.7309 & 0.0012 \\
\hline 5 & 2 & 4 & 4.5 & 4 & 1 & 3 & 3.5 & 12392.1229 & 0.0008 \\
\hline 5 & 2 & 4 & 6.5 & 4 & 1 & 3 & 5.5 & 12399.6717 & 0.0009 \\
\hline 5 & 2 & 4 & 3.5 & 4 & 1 & 3 & 2.5 & 12404.1025 & 0.0002 \\
\hline 7 & 2 & 5 & 8.5 & 6 & 2 & 4 & 7.5 & 12787.3312 & 0.0002 \\
\hline 7 & 2 & 5 & 5.5 & 6 & 2 & 4 & 4.5 & 12787.6020 & -0.0010 \\
\hline 7 & 2 & 5 & 7.5 & 6 & 2 & 4 & 6.5 & 12788.3752 & 0.0007 \\
\hline 7 & 2 & 5 & 6.5 & 6 & 2 & 4 & 5.5 & 12788.6535 & -0.0002 \\
\hline 8 & 2 & 7 & 8.5 & 7 & 2 & 6 & 7.5 & 13259.2850 & 0.0006 \\
\hline 8 & 2 & 7 & 9.5 & 7 & 2 & 6 & 8.5 & 13259.9494 & 0.0008 \\
\hline 8 & 2 & 7 & 6.5 & 7 & 2 & 6 & 5.5 & 13260.1310 & 0.0004 \\
\hline 4 & 2 & 2 & 2.5 & 3 & 1 & 3 & 1.5 & 13303.2599 & 0.0000 \\
\hline 4 & 2 & 2 & 5.5 & 3 & 1 & 3 & 4.5 & 13309.3070 & 0.0011 \\
\hline 4 & 2 & 2 & 3.5 & 3 & 1 & 3 & 2.5 & 13318.5014 & 0.0017 \\
\hline 4 & 2 & 2 & 4.5 & 3 & 1 & 3 & 3.5 & 13324.5490 & 0.0012 \\
\hline 6 & 2 & 5 & 6.5 & 5 & 1 & 4 & 5.5 & 13434.9259 & 0.0006 \\
\hline 6 & 2 & 5 & 5.5 & 5 & 1 & 4 & 4.5 & 13438.1178 & 0.0000 \\
\hline 6 & 2 & 5 & 7.5 & 5 & 1 & 4 & 6.5 & 13445.7893 & 0.0015 \\
\hline 6 & 2 & 5 & 4.5 & 5 & 1 & 4 & 3.5 & 13449.0446 & 0.0002 \\
\hline 9 & 1 & 9 & 8.5 & 8 & 1 & 8 & 7.5 & 13583.5757 & 0.0001 \\
\hline 9 & 1 & 9 & 9.5 & 8 & 1 & 8 & 8.5 & 13583.6440 & -0.0005 \\
\hline 9 & 1 & 9 & 7.5 & 8 & 1 & 8 & 6.5 & 13584.1233 & 0.0007 \\
\hline 9 & 1 & 9 & 10.5 & 8 & 1 & 8 & 9.5 & 13584.1900 & -0.0007 \\
\hline 9 & 0 & 9 & 8.5 & 8 & 0 & 8 & 7.5 & \multirow{2}{*}{13628.5975} & \multirow{2}{*}{0.0006} \\
\hline 9 & 0 & 9 & 9.5 & 8 & 0 & 8 & 8.5 & & \\
\hline 9 & 0 & 9 & 10.5 & 8 & 0 & 8 & 9.5 & 13629.4551 & 0.0011 \\
\hline 9 & 1 & 9 & 9.5 & 8 & 0 & 8 & 8.5 & 13682.0447 & 0.0010 \\
\hline 9 & 1 & 9 & 8.5 & 8 & 0 & 8 & 7.5 & 13682.0955 & 0.0010 \\
\hline 9 & 1 & 9 & 10.5 & 8 & 0 & 8 & 9.5 & 13683.2159 & 0.0006 \\
\hline 9 & 1 & 9 & 7.5 & 8 & 0 & 8 & 6.5 & 13683.2430 & -0.0022 \\
\hline 8 & 3 & 6 & 9.5 & 7 & 3 & 5 & 8.5 & 13780.4062 & 0.0009 \\
\hline
\end{tabular}


Table S3: Observed Transition Frequencies (in $\mathrm{MHz}$ ) for $\mathrm{CF}_{2} \mathrm{CH}^{37} \mathrm{Cl}-\mathrm{HCCH}$

\begin{tabular}{|c|c|c|c|c|c|c|c|c|c|}
\hline$J^{\prime}$ & $K_{a}^{\prime}$ & $K_{c}{ }^{\prime}$ & $F^{\prime}$ & $J^{\prime \prime}$ & $K_{a}^{\prime \prime}$ & $K_{c}^{\prime \prime}$ & $F^{\prime \prime}$ & Observed & Obs - Calc \\
\hline 8 & 3 & 6 & 6.5 & 7 & 3 & 5 & 5.5 & 13780.5164 & 0.0003 \\
\hline 8 & 3 & 6 & 7.5 & 7 & 3 & 5 & 6.5 & 13781.0715 & 0.0017 \\
\hline 8 & 3 & 6 & 8.5 & 7 & 3 & 5 & 7.5 & 13781.1058 & -0.0018 \\
\hline 3 & 3 & 1 & 3.5 & 2 & 2 & 0 & 2.5 & 13829.3895 & -0.0006 \\
\hline 3 & 3 & 1 & 2.5 & 2 & 2 & 0 & 1.5 & 13829.4727 & 0.0007 \\
\hline 3 & 3 & 1 & 4.5 & 2 & 2 & 0 & 3.5 & 13832.4103 & 0.0013 \\
\hline 3 & 3 & 1 & 1.5 & 2 & 2 & 0 & 0.5 & 13832.4380 & -0.0021 \\
\hline 3 & 3 & 1 & 2.5 & 2 & 2 & 0 & 2.5 & 13833.3164 & -0.0002 \\
\hline 8 & 1 & 7 & 8.5 & 7 & 1 & 6 & 7.5 & 13844.6973 & 0.0003 \\
\hline 8 & 1 & 7 & 7.5 & 7 & 1 & 6 & 6.5 & 13845.4631 & -0.0002 \\
\hline 8 & 1 & 7 & 9.5 & 7 & 1 & 6 & 8.5 & 13847.2575 & 0.0005 \\
\hline 8 & 1 & 7 & 6.5 & 7 & 1 & 6 & 5.5 & 13847.8580 & 0.0003 \\
\hline 3 & 3 & 0 & 3.5 & 2 & 2 & 1 & 3.5 & 13857.5210 & -0.0024 \\
\hline 3 & 3 & 0 & 2.5 & 2 & 2 & 1 & 1.5 & 13863.4128 & -0.0006 \\
\hline 3 & 3 & 0 & 3.5 & 2 & 2 & 1 & 2.5 & 13864.6432 & 0.0003 \\
\hline 3 & 3 & 0 & 4.5 & 2 & 2 & 1 & 3.5 & 13865.8317 & 0.0011 \\
\hline 3 & 3 & 0 & 2.5 & 2 & 2 & 1 & 2.5 & 13868.5235 & -0.0013 \\
\hline 8 & 3 & 5 & 6.5 & 7 & 3 & 4 & 5.5 & 14244.3559 & -0.0001 \\
\hline 8 & 3 & 5 & 9.5 & 7 & 3 & 4 & 8.5 & 14244.5963 & 0.0000 \\
\hline 8 & 3 & 5 & 7.5 & 7 & 3 & 4 & 6.5 & 14246.8813 & 0.0001 \\
\hline 8 & 3 & 5 & 8.5 & 7 & 3 & 4 & 7.5 & 14247.0902 & 0.0006 \\
\hline 7 & 2 & 6 & 7.5 & 6 & 1 & 5 & 6.5 & 14400.9837 & 0.0011 \\
\hline 7 & 2 & 6 & 6.5 & 6 & 1 & 5 & 5.5 & 14403.3277 & 0.0000 \\
\hline 7 & 2 & 6 & 8.5 & 6 & 1 & 5 & 7.5 & 14410.5943 & 0.0007 \\
\hline 7 & 2 & 6 & 5.5 & 6 & 1 & 5 & 4.5 & 14413.0098 & 0.0022 \\
\hline 8 & 2 & 6 & 9.5 & 7 & 2 & 5 & 8.5 & 14630.4864 & 0.0002 \\
\hline 8 & 2 & 6 & 8.5 & 7 & 2 & 5 & 7.5 & 14630.6570 & 0.0004 \\
\hline 8 & 2 & 6 & 6.5 & 7 & 2 & 5 & 5.5 & 14630.8453 & 0.0002 \\
\hline 8 & 2 & 6 & 7.5 & 7 & 2 & 5 & 6.5 & 14631.0227 & 0.0003 \\
\hline 9 & 2 & 8 & 9.5 & 8 & 2 & 7 & 8.5 & 14802.3170 & -0.0001 \\
\hline 9 & 2 & 8 & 8.5 & 8 & 2 & 7 & 7.5 & 14802.4941 & 0.0019 \\
\hline 9 & 2 & 8 & 10.5 & 8 & 2 & 7 & 9.5 & 14803.1295 & 0.0000 \\
\hline 9 & 2 & 8 & 7.5 & 8 & 2 & 7 & 6.5 & 14803.2986 & 0.0010 \\
\hline 10 & 1 & 10 & 9.5 & 9 & 1 & 9 & 8.5 & 15023.8844 & -0.0016 \\
\hline 10 & 1 & 10 & 10.5 & 9 & 1 & 9 & 9.5 & 15023.9511 & 0.0010 \\
\hline 10 & 1 & 10 & 8.5 & 9 & 1 & 9 & 7.5 & 15024.3703 & -0.0019 \\
\hline 10 & 0 & 10 & 9.5 & 9 & 0 & 9 & 8.5 & 15048.9588 & -0.0006 \\
\hline 10 & 0 & 10 & 10.5 & 9 & 0 & 9 & 9.5 & 15048.9942 & 0.0004 \\
\hline 10 & 0 & 10 & 8.5 & 9 & 0 & 9 & 7.5 & 15049.6014 & 0.0017 \\
\hline 10 & 0 & 10 & 11.5 & 9 & 0 & 9 & 10.5 & 15049.6393 & 0.0008 \\
\hline 9 & 1 & 8 & 9.5 & 8 & 1 & 7 & 8.5 & 15286.8050 & -0.0004 \\
\hline 9 & 1 & 8 & 8.5 & 8 & 1 & 7 & 7.5 & 15287.3647 & -0.0009 \\
\hline 9 & 1 & 8 & 10.5 & 8 & 1 & 7 & 9.5 & 15289.3356 & -0.0003 \\
\hline
\end{tabular}


Table S3: Observed Transition Frequencies (in $\mathrm{MHz}$ ) for $\mathrm{CF}_{2} \mathrm{CH}^{37} \mathrm{Cl}-\mathrm{HCCH}$

\begin{tabular}{|c|c|c|c|c|c|c|c|c|c|}
\hline$J^{\prime}$ & $K_{a}^{\prime}$ & $K_{c}^{\prime}$ & $F^{\prime}$ & $J^{\prime \prime}$ & $K_{a}^{\prime \prime}$ & $K_{c}^{\prime \prime}$ & $F^{\prime \prime}$ & Observed & Obs - Calc \\
\hline 9 & 1 & 8 & 7.5 & 8 & 1 & 7 & 6.5 & 15289.8807 & -0.0018 \\
\hline 4 & 3 & 2 & 4.5 & 3 & 2 & 1 & 3.5 & 15464.4254 & -0.0011 \\
\hline 4 & 3 & 2 & 3.5 & 3 & 2 & 1 & 2.5 & 15466.8575 & -0.0012 \\
\hline 4 & 3 & 2 & 5.5 & 3 & 2 & 1 & 4.5 & 15469.9175 & 0.0011 \\
\hline 4 & 3 & 2 & 2.5 & 3 & 2 & 1 & 1.5 & 15472.2834 & 0.0006 \\
\hline 9 & 3 & 7 & 10.5 & 8 & 3 & 6 & 9.5 & 15472.3612 & 0.0003 \\
\hline 9 & 3 & 7 & 7.5 & 8 & 3 & 6 & 6.5 & 15472.4419 & 0.0000 \\
\hline 9 & 3 & 7 & 9.5 & 8 & 3 & 6 & 8.5 & 15472.5663 & -0.0007 \\
\hline 9 & 3 & 7 & 8.5 & 8 & 3 & 6 & 7.5 & 15472.6608 & 0.0004 \\
\hline 4 & 3 & 1 & 4.5 & 3 & 2 & 2 & 3.5 & 15639.0472 & -0.0002 \\
\hline 4 & 3 & 1 & 3.5 & 3 & 2 & 2 & 2.5 & 15639.3213 & -0.0002 \\
\hline 4 & 3 & 1 & 5.5 & 3 & 2 & 2 & 4.5 & 15639.9485 & 0.0015 \\
\hline 4 & 3 & 1 & 2.5 & 3 & 2 & 2 & 1.5 & 15640.2929 & 0.0008 \\
\hline 5 & 2 & 3 & 3.5 & 4 & 1 & 4 & 2.5 & 16070.0236 & 0.0006 \\
\hline 5 & 2 & 3 & 6.5 & 4 & 1 & 4 & 5.5 & 16075.7459 & -0.0002 \\
\hline 5 & 2 & 3 & 4.5 & 4 & 1 & 4 & 3.5 & 16088.7017 & 0.0008 \\
\hline 5 & 2 & 3 & 5.5 & 4 & 1 & 4 & 4.5 & 16094.3512 & 0.0001 \\
\hline 9 & 3 & 6 & 7.5 & 8 & 3 & 5 & 6.5 & 16209.0993 & 0.0013 \\
\hline 9 & 3 & 6 & 10.5 & 8 & 3 & 5 & 9.5 & 16209.2153 & -0.0010 \\
\hline 9 & 3 & 6 & 8.5 & 8 & 3 & 5 & 7.5 & 16211.2457 & -0.0012 \\
\hline 9 & 3 & 6 & 9.5 & 8 & 3 & 5 & 8.5 & 16211.3608 & 0.0007 \\
\hline 9 & 2 & 7 & 9.5 & 8 & 2 & 6 & 8.5 & 16405.8550 & 0.0002 \\
\hline 9 & 2 & 7 & 8.5 & 8 & 2 & 6 & 7.5 & 16406.2608 & -0.0011 \\
\hline 9 & 2 & 7 & 10.5 & 8 & 2 & 6 & 9.5 & 16406.4065 & -0.0005 \\
\hline 9 & 2 & 7 & 7.5 & 8 & 2 & 6 & 6.5 & 16406.7942 & 0.0006 \\
\hline 10 & 1 & 9 & 10.5 & 9 & 1 & 8 & 9.5 & 16680.5720 & -0.0006 \\
\hline 10 & 1 & 9 & 9.5 & 9 & 1 & 8 & 8.5 & 16680.9855 & -0.0011 \\
\hline 10 & 1 & 9 & 11.5 & 9 & 1 & 8 & 10.5 & 16682.8591 & -0.0002 \\
\hline 10 & 1 & 9 & 8.5 & 9 & 1 & 8 & 7.5 & 16683.2665 & -0.0020 \\
\hline 5 & 3 & 3 & 5.5 & 4 & 2 & 2 & 4.5 & 16984.9972 & -0.0017 \\
\hline 5 & 3 & 3 & 4.5 & 4 & 2 & 2 & 3.5 & 16987.6482 & -0.0013 \\
\hline 5 & 3 & 3 & 6.5 & 4 & 2 & 2 & 5.5 & 16992.0625 & -0.0001 \\
\hline 5 & 3 & 3 & 3.5 & 4 & 2 & 2 & 2.5 & 16994.6819 & -0.0003 \\
\hline 5 & 3 & 2 & 3.5 & 4 & 2 & 3 & 2.5 & 17501.4936 & 0.0006 \\
\hline 5 & 3 & 2 & 6.5 & 4 & 2 & 3 & 5.5 & 17501.6739 & -0.0002 \\
\hline 5 & 3 & 2 & 5.5 & 4 & 2 & 3 & 4.5 & 17502.5103 & 0.0003 \\
\hline 5 & 3 & 2 & 4.5 & 4 & 2 & 3 & 3.5 & 17502.5319 & -0.0027 \\
\hline 4 & 4 & 1 & 3.5 & 3 & 3 & 0 & 2.5 & 19042.2406 & -0.0011 \\
\hline 4 & 4 & 1 & 4.5 & 3 & 3 & 0 & 3.5 & 19042.7668 & -0.0002 \\
\hline 4 & 4 & 1 & 2.5 & 3 & 3 & 0 & 1.5 & 19043.4985 & 0.0005 \\
\hline 4 & 4 & 1 & 5.5 & 3 & 3 & 0 & 4.5 & 19044.0266 & 0.0001 \\
\hline 4 & 4 & 0 & 3.5 & 3 & 3 & 1 & 2.5 & 19044.7220 & 0.0000 \\
\hline 4 & 4 & 0 & 4.5 & 3 & 3 & 1 & 3.5 & 19045.2941 & 0.0002 \\
\hline
\end{tabular}


Table S3: Observed Transition Frequencies (in $\mathrm{MHz}$ ) for $\mathrm{CF}_{2} \mathrm{CH}^{37} \mathrm{Cl}-\mathrm{HCCH}$

\begin{tabular}{|r|r|r|c|c|c|c|c|r|r|}
\hline$J^{\prime}$ & $K_{a}{ }^{\prime}$ & $K_{c}{ }^{\prime}$ & $F^{\prime}$ & $J^{\prime \prime}$ & $K_{a}^{\prime \prime}$ & $K_{c}{ }^{\prime \prime}$ & $F^{\prime \prime}$ & Observed & Obs - Calc \\
\hline 4 & 4 & 0 & 2.5 & 3 & 3 & 1 & 1.5 & 19045.8803 & 0.0020 \\
\hline 4 & 4 & 0 & 5.5 & 3 & 3 & 1 & 4.5 & 19046.4524 & 0.0005 \\
\hline
\end{tabular}


Table S4: Observed Transition Frequencies (in $\mathrm{MHz}$ ) for $\mathrm{CF}_{2} \mathrm{CH}^{35} \mathrm{Cl}-\mathrm{H}^{13} \mathrm{CCH}$

\begin{tabular}{|c|c|c|c|c|c|c|c|c|c|}
\hline$J^{\prime}$ & $K_{a}^{\prime}$ & $K_{c}{ }^{\prime}$ & $F^{\prime}$ & $J^{\prime \prime}$ & $K_{a}^{\prime \prime}$ & $K_{c}^{\prime \prime}$ & $F^{\prime \prime}$ & Observed & Obs - Calc \\
\hline 4 & 0 & 4 & 2.5 & 3 & 1 & 3 & 1.5 & 5266.0765 & 0.0003 \\
\hline 4 & 0 & 4 & 5.5 & 3 & 1 & 3 & 4.5 & 5270.0253 & -0.0004 \\
\hline 4 & 0 & 4 & 3.5 & 3 & 1 & 3 & 2.5 & 5274.5886 & 0.0029 \\
\hline 4 & 0 & 4 & 4.5 & 3 & 1 & 3 & 3.5 & 5278.3347 & 0.0011 \\
\hline 3 & 1 & 3 & 3.5 & 2 & 0 & 2 & 2.5 & 6092.2701 & -0.0010 \\
\hline 3 & 1 & 3 & 2.5 & 2 & 0 & 2 & 1.5 & 6100.7654 & -0.0014 \\
\hline 3 & 1 & 3 & 4.5 & 2 & 0 & 2 & 3.5 & 6106.7366 & 0.0007 \\
\hline 3 & 1 & 3 & 1.5 & 2 & 0 & 2 & 0.5 & 6115.3208 & 0.0020 \\
\hline 4 & 1 & 4 & 2.5 & 3 & 1 & 3 & 1.5 & 6141.6859 & -0.0008 \\
\hline 4 & 1 & 4 & 3.5 & 3 & 1 & 3 & 2.5 & 6141.8840 & -0.0017 \\
\hline 4 & 1 & 4 & 5.5 & 3 & 1 & 3 & 4.5 & 6142.6919 & 0.0001 \\
\hline 4 & 1 & 4 & 4.5 & 3 & 1 & 3 & 3.5 & 6142.7481 & -0.0012 \\
\hline 4 & 0 & 4 & 4.5 & 3 & 0 & 3 & 3.5 & 6449.8137 & -0.0010 \\
\hline 4 & 0 & 4 & 3.5 & 3 & 0 & 3 & 2.5 & 6451.5046 & -0.0002 \\
\hline 4 & 0 & 4 & 5.5 & 3 & 0 & 3 & 4.5 & 6453.1548 & -0.0008 \\
\hline 4 & 0 & 4 & 2.5 & 3 & 0 & 3 & 1.5 & 6454.7451 & 0.0016 \\
\hline 4 & 2 & 3 & 2.5 & 3 & 2 & 2 & 1.5 & 6681.4345 & 0.0001 \\
\hline 4 & 2 & 3 & 5.5 & 3 & 2 & 2 & 4.5 & 6682.0830 & -0.0011 \\
\hline 4 & 2 & 3 & 3.5 & 3 & 2 & 2 & 2.5 & 6683.8877 & -0.0001 \\
\hline 4 & 2 & 3 & 4.5 & 3 & 2 & 2 & 3.5 & 6685.6337 & -0.0005 \\
\hline 4 & 3 & 2 & 2.5 & 3 & 3 & 1 & 1.5 & 6746.1444 & -0.0016 \\
\hline 4 & 3 & 2 & 5.5 & 3 & 3 & 1 & 4.5 & 6750.5308 & -0.0017 \\
\hline 4 & 3 & 2 & 3.5 & 3 & 3 & 1 & 2.5 & 6755.1014 & 0.0002 \\
\hline 4 & 3 & 1 & 2.5 & 3 & 3 & 0 & 1.5 & 6756.4659 & 0.0005 \\
\hline 4 & 3 & 2 & 4.5 & 3 & 3 & 1 & 3.5 & 6759.4803 & -0.0004 \\
\hline 4 & 3 & 1 & 5.5 & 3 & 3 & 0 & 4.5 & 6761.0373 & -0.0007 \\
\hline 4 & 3 & 1 & 3.5 & 3 & 3 & 0 & 2.5 & 6765.8275 & 0.0003 \\
\hline 4 & 3 & 1 & 4.5 & 3 & 3 & 0 & 3.5 & 6770.2226 & -0.0002 \\
\hline 4 & 2 & 2 & 2.5 & 3 & 2 & 1 & 1.5 & 6931.9512 & 0.0017 \\
\hline 4 & 2 & 2 & 5.5 & 3 & 2 & 1 & 4.5 & 6933.6892 & 0.0007 \\
\hline 4 & 2 & 2 & 3.5 & 3 & 2 & 1 & 2.5 & 6938.4326 & 0.0010 \\
\hline 4 & 2 & 2 & 4.5 & 3 & 2 & 1 & 3.5 & 6940.1331 & -0.0012 \\
\hline 5 & 0 & 5 & 3.5 & 4 & 1 & 4 & 2.5 & 7041.4576 & 0.0010 \\
\hline 5 & 0 & 5 & 6.5 & 4 & 1 & 4 & 5.5 & 7042.7915 & 0.0008 \\
\hline 5 & 0 & 5 & 4.5 & 4 & 1 & 4 & 3.5 & 7045.9454 & -0.0013 \\
\hline 5 & 0 & 5 & 5.5 & 4 & 1 & 4 & 4.5 & 7048.1843 & 0.0005 \\
\hline 4 & 1 & 3 & 4.5 & 3 & 1 & 2 & 3.5 & 7157.9067 & 0.0001 \\
\hline 4 & 1 & 3 & 5.5 & 3 & 1 & 2 & 4.5 & 7158.1224 & 0.0002 \\
\hline 4 & 1 & 3 & 3.5 & 3 & 1 & 2 & 2.5 & 7160.2802 & -0.0006 \\
\hline 4 & 1 & 3 & 2.5 & 3 & 1 & 2 & 1.5 & 7160.4273 & -0.0004 \\
\hline 4 & 1 & 4 & 4.5 & 3 & 0 & 3 & 3.5 & 7314.2317 & 0.0012 \\
\hline 4 & 1 & 4 & 3.5 & 3 & 0 & 3 & 2.5 & 7318.8037 & -0.0011 \\
\hline 4 & 1 & 4 & 5.5 & 3 & 0 & 3 & 4.5 & 7325.8240 & 0.0023 \\
\hline
\end{tabular}


Table S4: Observed Transition Frequencies (in $\mathrm{MHz}$ ) for $\mathrm{CF}_{2} \mathrm{CH}^{35} \mathrm{Cl}-\mathrm{H}^{13} \mathrm{CCH}$

\begin{tabular}{|c|c|c|c|c|c|c|c|c|c|}
\hline$J^{\prime}$ & $K_{a}^{\prime}$ & $K_{c}{ }^{\prime}$ & $F^{\prime}$ & $J^{\prime \prime}$ & $K_{a}^{\prime \prime}$ & $K_{c}^{\prime \prime}$ & $F^{\prime \prime}$ & Observed & Obs - Calc \\
\hline 4 & 1 & 4 & 2.5 & 3 & 0 & 3 & 1.5 & 7330.3519 & -0.0021 \\
\hline 5 & 2 & 4 & 6.5 & 5 & 1 & 5 & 6.5 & 7503.5564 & -0.0034 \\
\hline 5 & 2 & 4 & 4.5 & 5 & 1 & 5 & 4.5 & 7512.1114 & 0.0011 \\
\hline 5 & 2 & 4 & 5.5 & 5 & 1 & 5 & 5.5 & 7515.5757 & -0.0001 \\
\hline 5 & 1 & 5 & 4.5 & 4 & 1 & 4 & 3.5 & 7638.0787 & -0.0004 \\
\hline 5 & 1 & 5 & 5.5 & 4 & 1 & 4 & 4.5 & 7638.5045 & -0.0005 \\
\hline 5 & 1 & 5 & 3.5 & 4 & 1 & 4 & 2.5 & 7638.6134 & -0.0004 \\
\hline 5 & 1 & 5 & 6.5 & 4 & 1 & 4 & 5.5 & 7638.8864 & -0.0006 \\
\hline 5 & 0 & 5 & 5.5 & 4 & 0 & 4 & 4.5 & 7912.5989 & -0.0006 \\
\hline 5 & 0 & 5 & 4.5 & 4 & 0 & 4 & 3.5 & 7913.2453 & -0.0013 \\
\hline 5 & 0 & 5 & 6.5 & 4 & 0 & 4 & 5.5 & 7915.4561 & -0.0008 \\
\hline 5 & 0 & 5 & 3.5 & 4 & 0 & 4 & 2.5 & 7917.0663 & -0.0008 \\
\hline 5 & 2 & 4 & 3.5 & 4 & 2 & 3 & 2.5 & 8320.4757 & 0.0011 \\
\hline 5 & 2 & 4 & 6.5 & 4 & 2 & 3 & 5.5 & 8320.8725 & -0.0003 \\
\hline 5 & 2 & 4 & 5.5 & 4 & 2 & 3 & 4.5 & 8321.7200 & -0.0010 \\
\hline 5 & 2 & 4 & 4.5 & 4 & 2 & 3 & 3.5 & 8322.0173 & -0.0012 \\
\hline 5 & 3 & 3 & 3.5 & 4 & 3 & 2 & 2.5 & 8452.7054 & -0.0003 \\
\hline 5 & 3 & 3 & 6.5 & 4 & 3 & 2 & 5.5 & 8454.1250 & -0.0004 \\
\hline 5 & 3 & 3 & 4.5 & 4 & 3 & 2 & 3.5 & 8457.2963 & -0.0001 \\
\hline 5 & 3 & 3 & 5.5 & 4 & 3 & 2 & 4.5 & 8458.7393 & -0.0005 \\
\hline 5 & 3 & 2 & 3.5 & 4 & 3 & 1 & 2.5 & 8488.5779 & 0.0005 \\
\hline 5 & 3 & 2 & 6.5 & 4 & 3 & 1 & 5.5 & 8490.0677 & -0.0008 \\
\hline 5 & 3 & 2 & 4.5 & 4 & 3 & 1 & 3.5 & 8493.7288 & 0.0001 \\
\hline 5 & 3 & 2 & 5.5 & 4 & 3 & 1 & 4.5 & 8495.4114 & -0.0004 \\
\hline 5 & 1 & 5 & 5.5 & 4 & 0 & 4 & 4.5 & 8502.9204 & -0.0003 \\
\hline 5 & 1 & 5 & 4.5 & 4 & 0 & 4 & 3.5 & 8505.3790 & -0.0001 \\
\hline 5 & 1 & 5 & 6.5 & 4 & 0 & 4 & 5.5 & 8511.5517 & -0.0014 \\
\hline 5 & 1 & 5 & 3.5 & 4 & 0 & 4 & 2.5 & 8514.2221 & -0.0022 \\
\hline 2 & 2 & 1 & 2.5 & 1 & 1 & 0 & 1.5 & 8708.8789 & -0.0004 \\
\hline 2 & 2 & 1 & 1.5 & 1 & 1 & 0 & 1.5 & 8715.3816 & -0.0018 \\
\hline 2 & 2 & 1 & 2.5 & 1 & 1 & 0 & 2.5 & 8717.9284 & -0.0016 \\
\hline 2 & 2 & 1 & 3.5 & 1 & 1 & 0 & 2.5 & 8726.9867 & 0.0017 \\
\hline 2 & 2 & 1 & 1.5 & 1 & 1 & 0 & 0.5 & 8731.7418 & -0.0010 \\
\hline 6 & 0 & 6 & 4.5 & 5 & 1 & 5 & 3.5 & 8734.1105 & 0.0004 \\
\hline 6 & 0 & 6 & 7.5 & 5 & 1 & 5 & 6.5 & 8735.0334 & 0.0002 \\
\hline 6 & 0 & 6 & 5.5 & 5 & 1 & 5 & 4.5 & 8736.7045 & -0.0002 \\
\hline 6 & 0 & 6 & 6.5 & 5 & 1 & 5 & 5.5 & 8737.7243 & -0.0005 \\
\hline 2 & 2 & 1 & 0.5 & 1 & 1 & 0 & 0.5 & 8740.7958 & -0.0024 \\
\hline 5 & 2 & 3 & 3.5 & 4 & 2 & 2 & 2.5 & 8784.2631 & 0.0007 \\
\hline 5 & 2 & 3 & 6.5 & 4 & 2 & 2 & 5.5 & 8784.8444 & 0.0016 \\
\hline 5 & 2 & 3 & 4.5 & 4 & 2 & 2 & 3.5 & 8788.7176 & -0.0006 \\
\hline 5 & 2 & 3 & 5.5 & 4 & 2 & 2 & 4.5 & 8789.0894 & 0.0001 \\
\hline 5 & 1 & 4 & 5.5 & 4 & 1 & 3 & 4.5 & 8884.4196 & -0.0002 \\
\hline
\end{tabular}


Table S4: Observed Transition Frequencies (in $\mathrm{MHz}$ ) for $\mathrm{CF}_{2} \mathrm{CH}^{35} \mathrm{Cl}-\mathrm{H}^{13} \mathrm{CCH}$

\begin{tabular}{|c|c|c|c|c|c|c|c|c|c|}
\hline$J^{\prime}$ & $K_{a}^{\prime}$ & $K_{c}^{\prime}$ & $F^{\prime}$ & $J^{\prime \prime}$ & $K_{a}^{\prime \prime}$ & $K_{c}^{\prime \prime}$ & $F^{\prime \prime}$ & Observed & Obs - Calc \\
\hline 5 & 1 & 4 & 6.5 & 4 & 1 & 3 & 5.5 & 8885.5273 & 0.0007 \\
\hline 5 & 1 & 4 & 4.5 & 4 & 1 & 3 & 3.5 & 8886.0800 & -0.0002 \\
\hline 5 & 1 & 4 & 3.5 & 4 & 1 & 3 & 2.5 & 8887.1689 & 0.0004 \\
\hline 2 & 2 & 0 & 1.5 & 1 & 1 & 1 & 0.5 & 8987.8578 & 0.0002 \\
\hline 2 & 2 & 0 & 0.5 & 1 & 1 & 1 & 0.5 & 8995.0665 & 0.0008 \\
\hline 2 & 2 & 0 & 2.5 & 1 & 1 & 1 & 2.5 & 8997.3885 & -0.0024 \\
\hline 2 & 2 & 0 & 3.5 & 1 & 1 & 1 & 2.5 & 9004.4588 & 0.0000 \\
\hline 2 & 2 & 0 & 2.5 & 1 & 1 & 1 & 1.5 & 9015.5138 & 0.0019 \\
\hline 2 & 2 & 0 & 1.5 & 1 & 1 & 1 & 1.5 & 9020.5406 & 0.0020 \\
\hline 7 & 1 & 6 & 5.5 & 6 & 2 & 5 & 4.5 & 9083.4536 & 0.0021 \\
\hline 7 & 1 & 6 & 8.5 & 6 & 2 & 5 & 7.5 & 9085.5046 & 0.0008 \\
\hline 7 & 1 & 6 & 6.5 & 6 & 2 & 5 & 5.5 & 9093.4776 & -0.0007 \\
\hline 7 & 1 & 6 & 7.5 & 6 & 2 & 5 & 6.5 & 9095.1895 & 0.0024 \\
\hline 6 & 1 & 6 & 5.5 & 5 & 1 & 5 & 4.5 & 9115.4303 & -0.0014 \\
\hline 6 & 1 & 6 & 6.5 & 5 & 1 & 5 & 5.5 & 9115.6166 & -0.0010 \\
\hline 6 & 1 & 6 & 4.5 & 5 & 1 & 5 & 3.5 & 9116.0915 & -0.0005 \\
\hline 6 & 1 & 6 & 7.5 & 5 & 1 & 5 & 6.5 & 9116.2963 & -0.0012 \\
\hline 6 & 0 & 6 & 6.5 & 5 & 0 & 5 & 5.5 & 9328.0446 & -0.0013 \\
\hline 6 & 0 & 6 & 5.5 & 5 & 0 & 5 & 4.5 & 9328.8363 & -0.0009 \\
\hline 6 & 0 & 6 & 7.5 & 5 & 0 & 5 & 6.5 & 9331.1284 & -0.0011 \\
\hline 6 & 0 & 6 & 4.5 & 5 & 0 & 5 & 3.5 & 9331.2665 & -0.0007 \\
\hline 6 & 1 & 6 & 6.5 & 5 & 0 & 5 & 5.5 & 9705.9386 & -0.0001 \\
\hline 6 & 1 & 6 & 5.5 & 5 & 0 & 5 & 4.5 & 9707.5634 & -0.0007 \\
\hline 6 & 1 & 6 & 7.5 & 5 & 0 & 5 & 6.5 & 9712.3928 & -0.0010 \\
\hline 6 & 1 & 6 & 4.5 & 5 & 0 & 5 & 3.5 & 9713.2493 & 0.0001 \\
\hline 6 & 2 & 5 & 7.5 & 5 & 2 & 4 & 6.5 & 9936.6416 & -0.0003 \\
\hline 6 & 2 & 5 & 6.5 & 5 & 2 & 4 & 5.5 & 9936.7739 & -0.0001 \\
\hline 6 & 2 & 5 & 4.5 & 5 & 2 & 4 & 3.5 & 9936.8068 & -0.0022 \\
\hline 6 & 2 & 5 & 5.5 & 5 & 2 & 4 & 4.5 & 9936.9526 & -0.0005 \\
\hline 3 & 2 & 2 & 3.5 & 2 & 1 & 1 & 2.5 & 10129.0815 & -0.0007 \\
\hline 3 & 2 & 2 & 2.5 & 2 & 1 & 1 & 1.5 & 10142.2390 & -0.0014 \\
\hline 6 & 4 & 3 & 4.5 & 5 & 4 & 2 & 3.5 & 10143.6579 & -0.0005 \\
\hline 6 & 4 & 3 & 7.5 & 5 & 4 & 2 & 6.5 & 10144.9819 & -0.0002 \\
\hline 6 & 4 & 2 & 4.5 & 5 & 4 & 1 & 3.5 & 10147.0422 & -0.0008 \\
\hline 3 & 2 & 2 & 4.5 & 2 & 1 & 1 & 3.5 & 10147.3108 & 0.0036 \\
\hline 6 & 4 & 2 & 7.5 & 5 & 4 & 1 & 6.5 & 10148.3904 & 0.0001 \\
\hline 6 & 4 & 3 & 6.5 & 5 & 4 & 2 & 5.5 & 10149.7101 & -0.0011 \\
\hline 6 & 4 & 2 & 5.5 & 5 & 4 & 1 & 4.5 & 10151.8656 & -0.0013 \\
\hline 6 & 4 & 2 & 6.5 & 5 & 4 & 1 & 5.5 & 10153.1787 & 0.0000 \\
\hline 6 & 3 & 4 & 4.5 & 5 & 3 & 3 & 3.5 & 10158.3758 & 0.0002 \\
\hline 6 & 3 & 4 & 7.5 & 5 & 3 & 3 & 6.5 & 10158.9829 & 0.0002 \\
\hline 3 & 2 & 2 & 1.5 & 2 & 1 & 1 & 0.5 & 10160.1623 & -0.0027 \\
\hline 6 & 3 & 4 & 5.5 & 5 & 3 & 3 & 4.5 & 10161.1676 & -0.0010 \\
\hline
\end{tabular}


Table S4: Observed Transition Frequencies (in $\mathrm{MHz}$ ) for $\mathrm{CF}_{2} \mathrm{CH}^{35} \mathrm{Cl}-\mathrm{H}^{13} \mathrm{CCH}$

\begin{tabular}{|c|c|c|c|c|c|c|c|c|c|}
\hline$J^{\prime}$ & $K_{a}^{\prime}$ & $K_{c}^{\prime}$ & $F^{\prime}$ & $J^{\prime \prime}$ & $K_{a}^{\prime \prime}$ & $K_{c}^{\prime \prime}$ & $F^{\prime \prime}$ & Observed & Obs - Calc \\
\hline 6 & 3 & 4 & 6.5 & 5 & 3 & 3 & 5.5 & 10161.5498 & 0.0000 \\
\hline 6 & 3 & 3 & 4.5 & 5 & 3 & 2 & 3.5 & 10251.6701 & 0.0006 \\
\hline 6 & 3 & 3 & 7.5 & 5 & 3 & 2 & 6.5 & 10252.4007 & -0.0007 \\
\hline 6 & 3 & 3 & 5.5 & 5 & 3 & 2 & 4.5 & 10255.4742 & 0.0003 \\
\hline 6 & 3 & 3 & 6.5 & 5 & 3 & 2 & 5.5 & 10256.1864 & -0.0005 \\
\hline 7 & 0 & 7 & 5.5 & 6 & 1 & 6 & 4.5 & 10344.9586 & -0.0003 \\
\hline 7 & 0 & 7 & 8.5 & 6 & 1 & 6 & 7.5 & 10345.4399 & 0.0002 \\
\hline 7 & 0 & 7 & 6.5 & 6 & 1 & 6 & 5.5 & 10346.0783 & -0.0001 \\
\hline 7 & 0 & 7 & 7.5 & 6 & 1 & 6 & 6.5 & 10346.6164 & -0.0005 \\
\hline 6 & 1 & 5 & 6.5 & 5 & 1 & 4 & 5.5 & 10560.2598 & -0.0004 \\
\hline 6 & 1 & 5 & 5.5 & 5 & 1 & 4 & 4.5 & 10561.5404 & -0.0005 \\
\hline 6 & 1 & 5 & 7.5 & 5 & 1 & 4 & 6.5 & 10562.0747 & 0.0000 \\
\hline 6 & 1 & 5 & 4.5 & 5 & 1 & 4 & 3.5 & 10563.3288 & -0.0001 \\
\hline 7 & 1 & 7 & 6.5 & 6 & 1 & 6 & 5.5 & 10576.1361 & -0.0004 \\
\hline 7 & 1 & 7 & 7.5 & 6 & 1 & 6 & 6.5 & 10576.2607 & -0.0005 \\
\hline 7 & 1 & 7 & 5.5 & 6 & 1 & 6 & 4.5 & 10576.8792 & -0.0010 \\
\hline 7 & 1 & 7 & 8.5 & 6 & 1 & 6 & 7.5 & 10577.0103 & -0.0001 \\
\hline 6 & 2 & 4 & 7.5 & 5 & 2 & 3 & 6.5 & 10658.5278 & 0.0003 \\
\hline 6 & 2 & 4 & 4.5 & 5 & 2 & 3 & 3.5 & 10658.6717 & -0.0010 \\
\hline 6 & 2 & 4 & 5.5 & 5 & 2 & 3 & 4.5 & 10661.4425 & -0.0006 \\
\hline 6 & 2 & 4 & 6.5 & 5 & 2 & 3 & 5.5 & 10661.4937 & 0.0014 \\
\hline 7 & 0 & 7 & 7.5 & 6 & 0 & 6 & 6.5 & 10724.5086 & -0.0011 \\
\hline 7 & 0 & 7 & 6.5 & 6 & 0 & 6 & 5.5 & 10724.8037 & -0.0016 \\
\hline 7 & 0 & 7 & 8.5 & 6 & 0 & 6 & 7.5 & 10726.7031 & -0.0009 \\
\hline 7 & 0 & 7 & 5.5 & 6 & 0 & 6 & 4.5 & 10726.9394 & -0.0014 \\
\hline 7 & 1 & 7 & 7.5 & 6 & 0 & 6 & 6.5 & 10954.1530 & -0.0010 \\
\hline 7 & 1 & 7 & 6.5 & 6 & 0 & 6 & 5.5 & 10954.8618 & -0.0016 \\
\hline 7 & 1 & 7 & 8.5 & 6 & 0 & 6 & 7.5 & 10958.2734 & -0.0012 \\
\hline 7 & 1 & 7 & 5.5 & 6 & 0 & 6 & 4.5 & 10958.8609 & -0.0012 \\
\hline 3 & 2 & 1 & 1.5 & 2 & 1 & 2 & 0.5 & 11031.5346 & 0.0002 \\
\hline 3 & 2 & 1 & 4.5 & 2 & 1 & 2 & 3.5 & 11040.2898 & -0.0003 \\
\hline 3 & 2 & 1 & 2.5 & 2 & 1 & 2 & 1.5 & 11045.3364 & -0.0004 \\
\hline 3 & 2 & 1 & 3.5 & 2 & 1 & 2 & 2.5 & 11054.1126 & 0.0016 \\
\hline 8 & 1 & 7 & 6.5 & 7 & 2 & 6 & 5.5 & 11261.9033 & 0.0019 \\
\hline 8 & 1 & 7 & 9.5 & 7 & 2 & 6 & 8.5 & 11263.0941 & 0.0011 \\
\hline 8 & 1 & 7 & 7.5 & 7 & 2 & 6 & 6.5 & 11269.2453 & 0.0025 \\
\hline 8 & 1 & 7 & 8.5 & 7 & 2 & 6 & 7.5 & 11270.4724 & 0.0012 \\
\hline 4 & 2 & 3 & 4.5 & 3 & 1 & 2 & 3.5 & 11418.6604 & 0.0021 \\
\hline 4 & 2 & 3 & 3.5 & 3 & 1 & 2 & 2.5 & 11426.3212 & -0.0021 \\
\hline 4 & 2 & 3 & 5.5 & 3 & 1 & 2 & 4.5 & 11434.9637 & 0.0028 \\
\hline 4 & 2 & 3 & 2.5 & 3 & 1 & 2 & 1.5 & 11443.3749 & -0.0016 \\
\hline 7 & 2 & 6 & 7.5 & 6 & 2 & 5 & 6.5 & 11527.2211 & -0.0005 \\
\hline 7 & 2 & 6 & 6.5 & 6 & 2 & 5 & 5.5 & 11527.4714 & -0.0003 \\
\hline
\end{tabular}


Table S4: Observed Transition Frequencies (in $\mathrm{MHz}$ ) for $\mathrm{CF}_{2} \mathrm{CH}^{35} \mathrm{Cl}-\mathrm{H}^{13} \mathrm{CCH}$

\begin{tabular}{|c|c|c|c|c|c|c|c|c|c|}
\hline$J^{\prime}$ & $K_{a}{ }^{\prime}$ & $K_{c}{ }^{\prime}$ & $F^{\prime}$ & $J^{\prime \prime}$ & $K_{a}^{\prime \prime}$ & $K_{c}^{\prime \prime}$ & $F^{\prime \prime}$ & Observed & Obs - Calc \\
\hline 7 & 2 & 6 & 8.5 & 6 & 2 & 5 & 7.5 & 11527.6492 & 0.0002 \\
\hline 7 & 2 & 6 & 5.5 & 6 & 2 & 5 & 4.5 & 11527.8425 & 0.0002 \\
\hline 7 & 3 & 5 & 5.5 & 6 & 3 & 4 & 4.5 & 11859.5183 & 0.0007 \\
\hline 7 & 3 & 5 & 8.5 & 6 & 3 & 4 & 7.5 & 11859.5334 & 0.0010 \\
\hline 7 & 4 & 4 & 5.5 & 6 & 4 & 3 & 4.5 & 11859.9072 & 0.0024 \\
\hline 7 & 4 & 4 & 8.5 & 6 & 4 & 3 & 7.5 & 11860.5125 & 0.0003 \\
\hline 7 & 3 & 5 & 6.5 & 6 & 3 & 4 & 5.5 & 11860.9354 & 0.0003 \\
\hline 7 & 3 & 5 & 7.5 & 6 & 3 & 4 & 6.5 & 11861.2471 & 0.0003 \\
\hline 7 & 4 & 4 & 6.5 & 6 & 4 & 3 & 5.5 & 11863.0111 & 0.0002 \\
\hline 7 & 4 & 4 & 7.5 & 6 & 4 & 3 & 6.5 & 11863.6540 & -0.0002 \\
\hline 7 & 4 & 3 & 5.5 & 6 & 4 & 2 & 4.5 & 11871.0658 & -0.0013 \\
\hline 7 & 4 & 3 & 8.5 & 6 & 4 & 2 & 7.5 & 11871.7012 & -0.0001 \\
\hline 7 & 4 & 3 & 6.5 & 6 & 4 & 2 & 5.5 & 11874.3269 & 0.0007 \\
\hline 7 & 4 & 3 & 7.5 & 6 & 4 & 2 & 6.5 & 11874.9988 & -0.0001 \\
\hline 8 & 0 & 8 & 6.5 & 7 & 1 & 7 & 5.5 & 11888.6396 & 0.0000 \\
\hline 8 & 0 & 8 & 9.5 & 7 & 1 & 7 & 8.5 & 11888.8976 & -0.0001 \\
\hline 8 & 0 & 8 & 7.5 & 7 & 1 & 7 & 6.5 & 11888.9176 & 0.0032 \\
\hline 8 & 0 & 8 & 8.5 & 7 & 1 & 7 & 7.5 & 11889.2124 & -0.0002 \\
\hline 8 & 1 & 8 & 7.5 & 7 & 1 & 7 & 6.5 & 12023.3507 & 0.0006 \\
\hline 8 & 1 & 8 & 8.5 & 7 & 1 & 7 & 7.5 & 12023.4443 & 0.0000 \\
\hline 8 & 1 & 8 & 6.5 & 7 & 1 & 7 & 5.5 & 12024.0889 & -0.0001 \\
\hline 8 & 1 & 8 & 9.5 & 7 & 1 & 7 & 8.5 & 12024.1852 & 0.0002 \\
\hline 7 & 3 & 4 & 6.5 & 6 & 3 & 3 & 5.5 & 12062.8904 & 0.0006 \\
\hline 8 & 0 & 8 & 8.5 & 7 & 0 & 7 & 7.5 & 12118.8570 & 0.0001 \\
\hline 8 & 0 & 8 & 7.5 & 7 & 0 & 7 & 6.5 & 12118.9723 & -0.0002 \\
\hline 8 & 0 & 8 & 9.5 & 7 & 0 & 7 & 8.5 & 12120.4687 & 0.0003 \\
\hline 8 & 0 & 8 & 6.5 & 7 & 0 & 7 & 5.5 & 12120.5605 & -0.0004 \\
\hline 7 & 1 & 6 & 7.5 & 6 & 1 & 5 & 6.5 & 12169.7534 & -0.0002 \\
\hline 7 & 1 & 6 & 6.5 & 6 & 1 & 5 & 5.5 & 12170.8711 & -0.0001 \\
\hline 7 & 1 & 6 & 8.5 & 6 & 1 & 5 & 7.5 & 12172.2560 & 0.0002 \\
\hline 7 & 1 & 6 & 5.5 & 6 & 1 & 5 & 4.5 & 12173.1856 & -0.0007 \\
\hline 8 & 1 & 8 & 8.5 & 7 & 0 & 7 & 7.5 & 12253.0884 & -0.0002 \\
\hline 8 & 1 & 8 & 7.5 & 7 & 0 & 7 & 6.5 & 12253.4082 & 0.0001 \\
\hline 8 & 1 & 8 & 9.5 & 7 & 0 & 7 & 8.5 & 12255.7570 & 0.0013 \\
\hline 8 & 1 & 8 & 6.5 & 7 & 0 & 7 & 5.5 & 12256.0107 & 0.0004 \\
\hline 7 & 2 & 5 & 8.5 & 6 & 2 & 4 & 7.5 & 12522.0170 & 0.0015 \\
\hline 7 & 2 & 5 & 5.5 & 6 & 2 & 4 & 4.5 & 12522.2847 & 0.0008 \\
\hline 7 & 2 & 5 & 7.5 & 6 & 2 & 4 & 6.5 & 12523.6205 & 0.0004 \\
\hline 7 & 2 & 5 & 6.5 & 6 & 2 & 4 & 5.5 & 12523.9023 & 0.0008 \\
\hline 5 & 2 & 4 & 5.5 & 4 & 1 & 3 & 4.5 & 12582.4756 & 0.0030 \\
\hline 5 & 2 & 4 & 4.5 & 4 & 1 & 3 & 3.5 & 12588.0624 & 0.0014 \\
\hline 5 & 2 & 4 & 6.5 & 4 & 1 & 3 & 5.5 & 12597.7159 & 0.0045 \\
\hline 5 & 2 & 4 & 3.5 & 4 & 1 & 3 & 2.5 & 12603.4235 & 0.0002 \\
\hline
\end{tabular}


Table S4: Observed Transition Frequencies (in $\mathrm{MHz}$ ) for $\mathrm{CF}_{2} \mathrm{CH}^{35} \mathrm{Cl}-\mathrm{H}^{13} \mathrm{CCH}$

\begin{tabular}{|c|c|c|c|c|c|c|c|c|c|}
\hline$J^{\prime}$ & $K_{a}{ }^{\prime}$ & $K_{c}{ }^{\prime}$ & $F^{\prime}$ & $J^{\prime \prime}$ & $K_{a}^{\prime \prime}$ & $K_{c}^{\prime \prime}$ & $F^{\prime \prime}$ & Observed & Obs - Calc \\
\hline 8 & 2 & 7 & 8.5 & 7 & 2 & 6 & 7.5 & 13090.9450 & 0.0002 \\
\hline 8 & 2 & 7 & 7.5 & 7 & 2 & 6 & 6.5 & 13091.1891 & 0.0007 \\
\hline 8 & 2 & 7 & 9.5 & 7 & 2 & 6 & 8.5 & 13091.6860 & 0.0010 \\
\hline 8 & 2 & 7 & 6.5 & 7 & 2 & 6 & 5.5 & 13091.9061 & 0.0001 \\
\hline 4 & 2 & 2 & 2.5 & 3 & 1 & 3 & 1.5 & 13339.1583 & 0.0003 \\
\hline 4 & 2 & 2 & 5.5 & 3 & 1 & 3 & 4.5 & 13346.5663 & -0.0019 \\
\hline 4 & 2 & 2 & 3.5 & 3 & 1 & 3 & 2.5 & 13357.7486 & 0.0006 \\
\hline 4 & 2 & 2 & 4.5 & 3 & 1 & 3 & 3.5 & 13365.1586 & -0.0009 \\
\hline 9 & 0 & 9 & 8.5 & 8 & 1 & 8 & 7.5 & 13384.1640 & 0.0010 \\
\hline 9 & 0 & 9 & 7.5 & 8 & 1 & 8 & 6.5 & 13384.3211 & -0.0001 \\
\hline 9 & 0 & 9 & 9.5 & 8 & 1 & 8 & 8.5 & 13384.3450 & -0.0002 \\
\hline 9 & 0 & 9 & 10.5 & 8 & 1 & 8 & 9.5 & 13384.4744 & 0.0001 \\
\hline 9 & 1 & 9 & 8.5 & 8 & 1 & 8 & 7.5 & 13460.4713 & 0.0005 \\
\hline 9 & 1 & 9 & 9.5 & 8 & 1 & 8 & 8.5 & 13460.5523 & -0.0002 \\
\hline 9 & 1 & 9 & 7.5 & 8 & 1 & 8 & 6.5 & 13461.1626 & 0.0003 \\
\hline 9 & 1 & 9 & 10.5 & 8 & 1 & 8 & 9.5 & 13461.2435 & 0.0000 \\
\hline 9 & 0 & 9 & 9.5 & 8 & 0 & 8 & 8.5 & 13518.5777 & 0.0008 \\
\hline 9 & 0 & 9 & 8.5 & 8 & 0 & 8 & 7.5 & 13518.5956 & -0.0030 \\
\hline 9 & 0 & 9 & 10.5 & 8 & 0 & 8 & 9.5 & 13519.7597 & -0.0019 \\
\hline 9 & 0 & 9 & 7.5 & 8 & 0 & 8 & 6.5 & 13519.7747 & 0.0040 \\
\hline 8 & 3 & 6 & 9.5 & 7 & 3 & 5 & 8.5 & 13550.2212 & 0.0008 \\
\hline 8 & 3 & 6 & 8.5 & 7 & 3 & 5 & 7.5 & 13551.1120 & 0.0001 \\
\hline 8 & 3 & 6 & 7.5 & 7 & 3 & 5 & 6.5 & 13551.1341 & -0.0019 \\
\hline 9 & 1 & 9 & 9.5 & 8 & 0 & 8 & 8.5 & 13594.7847 & 0.0006 \\
\hline 9 & 1 & 9 & 8.5 & 8 & 0 & 8 & 7.5 & 13594.9058 & -0.0006 \\
\hline 9 & 1 & 9 & 10.5 & 8 & 0 & 8 & 9.5 & 13596.5317 & 0.0009 \\
\hline 9 & 1 & 9 & 7.5 & 8 & 0 & 8 & 6.5 & 13596.6111 & -0.0006 \\
\hline 6 & 2 & 5 & 6.5 & 5 & 1 & 4 & 5.5 & 13634.8296 & 0.0029 \\
\hline 6 & 2 & 5 & 5.5 & 5 & 1 & 4 & 4.5 & 13638.9359 & 0.0020 \\
\hline 6 & 2 & 5 & 7.5 & 5 & 1 & 4 & 6.5 & 13648.8295 & 0.0028 \\
\hline 6 & 2 & 5 & 4.5 & 5 & 1 & 4 & 3.5 & 13653.0660 & 0.0022 \\
\hline 8 & 1 & 7 & 8.5 & 7 & 1 & 6 & 7.5 & 13702.5050 & -0.0007 \\
\hline 8 & 1 & 7 & 7.5 & 7 & 1 & 6 & 6.5 & 13703.2361 & -0.0001 \\
\hline 8 & 1 & 7 & 9.5 & 7 & 1 & 6 & 8.5 & 13705.2379 & -0.0003 \\
\hline 8 & 1 & 7 & 6.5 & 7 & 1 & 6 & 5.5 & 13706.2914 & -0.0009 \\
\hline 8 & 3 & 5 & 6.5 & 7 & 3 & 4 & 5.5 & 13920.1708 & 0.0012 \\
\hline 8 & 3 & 5 & 9.5 & 7 & 3 & 4 & 8.5 & 13920.4175 & 0.0007 \\
\hline 8 & 3 & 5 & 7.5 & 7 & 3 & 4 & 6.5 & 13923.1636 & -0.0001 \\
\hline 8 & 3 & 5 & 8.5 & 7 & 3 & 4 & 7.5 & 13923.4546 & 0.0005 \\
\hline 3 & 3 & 1 & 2.5 & 2 & 2 & 0 & 1.5 & \multirow{2}{*}{14179.1606} & \multirow{2}{*}{0.0006} \\
\hline 3 & 3 & 1 & 3.5 & 2 & 2 & 0 & 2.5 & & \\
\hline 3 & 3 & 1 & 1.5 & 2 & 2 & 0 & 0.5 & 14182.7324 & 0.0036 \\
\hline 3 & 3 & 1 & 4.5 & 2 & 2 & 0 & 3.5 & 14182.8465 & 0.0006 \\
\hline
\end{tabular}


Table S4: Observed Transition Frequencies (in $\mathrm{MHz}$ ) for $\mathrm{CF}_{2} \mathrm{CH}^{35} \mathrm{Cl}-\mathrm{H}^{13} \mathrm{CCH}$

\begin{tabular}{|c|c|c|c|c|c|c|c|c|c|}
\hline$J^{\prime}$ & $K_{a}^{\prime}$ & $K_{c}{ }^{\prime}$ & $F^{\prime}$ & $J^{\prime \prime}$ & $K_{a}^{\prime \prime}$ & $K_{c}^{\prime \prime}$ & $F^{\prime \prime}$ & Observed & Obs - Calc \\
\hline 3 & 3 & 0 & 2.5 & 2 & 2 & 1 & 1.5 & 14207.7114 & 0.0004 \\
\hline 3 & 3 & 0 & 3.5 & 2 & 2 & 1 & 2.5 & 14209.2359 & -0.0002 \\
\hline 3 & 3 & 0 & 1.5 & 2 & 2 & 1 & 0.5 & 14209.3413 & 0.0045 \\
\hline 3 & 3 & 0 & 4.5 & 2 & 2 & 1 & 3.5 & 14210.8349 & 0.0006 \\
\hline 8 & 2 & 6 & 9.5 & 7 & 2 & 5 & 8.5 & 14349.0209 & 0.0015 \\
\hline 8 & 2 & 6 & 6.5 & 7 & 2 & 5 & 5.5 & 14349.4096 & 0.0013 \\
\hline 8 & 2 & 6 & 8.5 & 7 & 2 & 5 & 7.5 & 14349.5737 & 0.0003 \\
\hline 8 & 2 & 6 & 7.5 & 7 & 2 & 5 & 6.5 & 14349.9717 & 0.0006 \\
\hline 7 & 2 & 6 & 7.5 & 6 & 1 & 5 & 6.5 & 14601.7904 & 0.0023 \\
\hline 7 & 2 & 6 & 6.5 & 6 & 1 & 5 & 5.5 & 14604.8678 & 0.0031 \\
\hline 7 & 2 & 6 & 8.5 & 6 & 1 & 5 & 7.5 & 14614.4034 & 0.0024 \\
\hline 7 & 2 & 6 & 5.5 & 6 & 1 & 5 & 4.5 & 14617.5780 & 0.0008 \\
\hline 9 & 2 & 8 & 9.5 & 8 & 2 & 7 & 8.5 & 14627.2810 & 0.0003 \\
\hline 9 & 2 & 8 & 8.5 & 8 & 2 & 7 & 7.5 & 14627.4981 & -0.0005 \\
\hline 9 & 2 & 8 & 10.5 & 8 & 2 & 7 & 9.5 & 14628.2204 & 0.0012 \\
\hline 9 & 2 & 8 & 7.5 & 8 & 2 & 7 & 6.5 & 14628.4238 & -0.0003 \\
\hline 10 & 0 & 10 & 9.5 & 9 & 1 & 9 & 8.5 & 14848.1988 & 0.0002 \\
\hline 10 & 0 & 10 & 10.5 & 9 & 1 & 9 & 9.5 & 14848.3240 & 0.0000 \\
\hline 10 & 0 & 10 & 8.5 & 9 & 1 & 9 & 7.5 & 14848.5493 & -0.0002 \\
\hline 10 & 0 & 10 & 11.5 & 9 & 1 & 9 & 10.5 & 14848.6546 & 0.0003 \\
\hline 10 & 1 & 10 & 9.5 & 9 & 1 & 9 & 8.5 & 14890.5612 & 0.0010 \\
\hline 10 & 1 & 10 & 10.5 & 9 & 1 & 9 & 9.5 & 14890.6353 & -0.0007 \\
\hline 10 & 1 & 10 & 8.5 & 9 & 1 & 9 & 7.5 & 14891.1855 & 0.0002 \\
\hline 10 & 1 & 10 & 11.5 & 9 & 1 & 9 & 10.5 & 14891.2591 & -0.0003 \\
\hline 10 & 0 & 10 & 9.5 & 9 & 0 & 9 & 8.5 & 14924.5059 & -0.0004 \\
\hline 10 & 0 & 10 & 10.5 & 9 & 0 & 9 & 9.5 & 14924.5293 & -0.0019 \\
\hline 9 & 1 & 8 & 9.5 & 8 & 1 & 7 & 8.5 & 15159.9186 & -0.0010 \\
\hline 9 & 1 & 8 & 8.5 & 8 & 1 & 7 & 7.5 & 15160.6479 & -0.0006 \\
\hline 9 & 1 & 8 & 10.5 & 8 & 1 & 7 & 9.5 & 15163.0067 & -0.0007 \\
\hline 9 & 1 & 8 & 7.5 & 8 & 1 & 7 & 6.5 & 15163.6853 & -0.0013 \\
\hline 9 & 3 & 7 & 10.5 & 8 & 3 & 6 & 9.5 & 15223.9834 & 0.0010 \\
\hline 9 & 3 & 7 & 9.5 & 8 & 3 & 6 & 8.5 & 15224.3530 & -0.0001 \\
\hline 9 & 3 & 7 & 8.5 & 8 & 3 & 6 & 7.5 & 15224.4569 & 0.0005 \\
\hline 8 & 2 & 7 & 8.5 & 7 & 1 & 6 & 7.5 & 15522.9822 & 0.0029 \\
\hline 8 & 2 & 7 & 7.5 & 7 & 1 & 6 & 6.5 & 15525.1843 & 0.0024 \\
\hline 8 & 2 & 7 & 9.5 & 7 & 1 & 6 & 8.5 & 15533.8323 & 0.0021 \\
\hline 8 & 2 & 7 & 6.5 & 7 & 1 & 6 & 5.5 & 15536.2950 & -0.0018 \\
\hline 4 & 3 & 2 & 4.5 & 3 & 2 & 1 & 3.5 & 15796.2712 & -0.0016 \\
\hline 4 & 3 & 2 & 3.5 & 3 & 2 & 1 & 2.5 & 15799.1646 & -0.0019 \\
\hline 4 & 3 & 2 & 5.5 & 3 & 2 & 1 & 4.5 & 15802.8709 & 0.0001 \\
\hline 4 & 3 & 2 & 2.5 & 3 & 2 & 1 & 1.5 & 15805.6909 & -0.0019 \\
\hline 9 & 3 & 6 & 7.5 & 8 & 3 & 5 & 6.5 & 15827.3596 & 0.0006 \\
\hline 9 & 3 & 6 & 10.5 & 8 & 3 & 5 & 9.5 & 15827.5320 & 0.0002 \\
\hline
\end{tabular}


Table S4: Observed Transition Frequencies (in $\mathrm{MHz}$ ) for $\mathrm{CF}_{2} \mathrm{CH}^{35} \mathrm{Cl}-\mathrm{H}^{13} \mathrm{CCH}$

\begin{tabular}{|c|c|c|c|c|c|c|c|c|c|}
\hline$J^{\prime}$ & $K_{a}^{\prime}$ & $K_{c}^{\prime}$ & $F^{\prime}$ & $J^{\prime \prime}$ & $K_{a}^{\prime \prime}$ & $K_{c}^{\prime \prime}$ & $F^{\prime \prime}$ & Observed & Obs - Calc \\
\hline 9 & 3 & 6 & 8.5 & 8 & 3 & 5 & 7.5 & 15830.0915 & 0.0006 \\
\hline 9 & 3 & 6 & 9.5 & 8 & 3 & 5 & 8.5 & 15830.2518 & 0.0003 \\
\hline 4 & 3 & 1 & 4.5 & 3 & 2 & 2 & 3.5 & 15944.3961 & -0.0018 \\
\hline 4 & 3 & 1 & 3.5 & 3 & 2 & 2 & 2.5 & 15944.8251 & -0.0019 \\
\hline 4 & 3 & 1 & 3.5 & 3 & 2 & 2 & 3.5 & 15944.9812 & 0.0000 \\
\hline 4 & 3 & 1 & 5.5 & 3 & 2 & 2 & 4.5 & 15945.8071 & -0.0004 \\
\hline 4 & 3 & 1 & 2.5 & 3 & 2 & 2 & 1.5 & 15946.3050 & -0.0013 \\
\hline 9 & 2 & 7 & 9.5 & 8 & 2 & 6 & 8.5 & 16121.5390 & -0.0005 \\
\hline 9 & 2 & 7 & 10.5 & 8 & 2 & 6 & 9.5 & 16121.8565 & 0.0000 \\
\hline 9 & 2 & 7 & 8.5 & 8 & 2 & 6 & 7.5 & 16121.9908 & -0.0016 \\
\hline 9 & 2 & 7 & 7.5 & 8 & 2 & 6 & 6.5 & 16122.2956 & 0.0004 \\
\hline 10 & 2 & 9 & 10.5 & 9 & 2 & 8 & 9.5 & 16137.3238 & -0.0013 \\
\hline 10 & 2 & 9 & 9.5 & 9 & 2 & 8 & 8.5 & 16137.5143 & -0.0017 \\
\hline 10 & 2 & 9 & 11.5 & 9 & 2 & 8 & 10.5 & 16138.3769 & 0.0025 \\
\hline 10 & 2 & 9 & 8.5 & 9 & 2 & 8 & 7.5 & 16138.5560 & -0.0010 \\
\hline 11 & 1 & 11 & 10.5 & 10 & 1 & 10 & 9.5 & 16316.0325 & 0.0013 \\
\hline 11 & 1 & 11 & 11.5 & 10 & 1 & 10 & 10.5 & 16316.1049 & 0.0022 \\
\hline 11 & 1 & 11 & 12.5 & 10 & 1 & 10 & 11.5 & 16316.6545 & -0.0004 \\
\hline 11 & 0 & 11 & 10.5 & 10 & 0 & 10 & 9.5 & 16335.2842 & 0.0013 \\
\hline 11 & 0 & 11 & 11.5 & 10 & 0 & 10 & 10.5 & 16335.3287 & -0.0007 \\
\hline 11 & 0 & 11 & 9.5 & 10 & 0 & 10 & 8.5 & 16335.9719 & -0.0002 \\
\hline 10 & 1 & 9 & 10.5 & 9 & 1 & 8 & 9.5 & 16561.2372 & -0.0018 \\
\hline 10 & 1 & 9 & 9.5 & 9 & 1 & 8 & 8.5 & 16561.7920 & -0.0031 \\
\hline 10 & 1 & 9 & 11.5 & 9 & 1 & 8 & 10.5 & 16564.1722 & -0.0008 \\
\hline 10 & 1 & 9 & 8.5 & 9 & 1 & 8 & 7.5 & 16564.7159 & -0.0005 \\
\hline 5 & 3 & 3 & 5.5 & 4 & 2 & 2 & 4.5 & 17314.8770 & -0.0012 \\
\hline 5 & 3 & 3 & 4.5 & 4 & 2 & 2 & 3.5 & 17318.0293 & -0.0019 \\
\hline 5 & 3 & 3 & 6.5 & 4 & 2 & 2 & 5.5 & 17323.3072 & -0.0004 \\
\hline 5 & 3 & 3 & 3.5 & 4 & 2 & 2 & 2.5 & 17326.4493 & 0.0003 \\
\hline 5 & 3 & 2 & 3.5 & 4 & 2 & 3 & 2.5 & 17753.4477 & -0.0017 \\
\hline 5 & 3 & 2 & 6.5 & 4 & 2 & 3 & 5.5 & 17753.7913 & -0.0007 \\
\hline 5 & 3 & 2 & 5.5 & 4 & 2 & 3 & 4.5 & 17754.1746 & -0.0009 \\
\hline 5 & 3 & 2 & 4.5 & 4 & 2 & 3 & 3.5 & 17754.6666 & -0.0014 \\
\hline 10 & 2 & 8 & 10.5 & 9 & 2 & 7 & 9.5 & 17825.9020 & -0.0022 \\
\hline 10 & 2 & 8 & 9.5 & 9 & 2 & 7 & 8.5 & 17826.4126 & -0.0031 \\
\hline 10 & 2 & 8 & 11.5 & 9 & 2 & 7 & 10.5 & 17827.0010 & -0.0005 \\
\hline 4 & 4 & 1 & 3.5 & 3 & 3 & 0 & 2.5 & 19534.3455 & -0.0011 \\
\hline 4 & 4 & 1 & 4.5 & 3 & 3 & 0 & 3.5 & 19535.0340 & -0.0017 \\
\hline 4 & 4 & 1 & 2.5 & 3 & 3 & 0 & 1.5 & 19535.9130 & 0.0012 \\
\hline 4 & 4 & 0 & 3.5 & 3 & 3 & 1 & 2.5 & 19536.2299 & -0.0014 \\
\hline 4 & 4 & 1 & 5.5 & 3 & 3 & 0 & 4.5 & 19536.6081 & 0.0004 \\
\hline 4 & 4 & 0 & 4.5 & 3 & 3 & 1 & 3.5 & 19536.9671 & -0.0007 \\
\hline 4 & 4 & 0 & 2.5 & 3 & 3 & 1 & 1.5 & 19537.6999 & 0.0045 \\
\hline
\end{tabular}


Table S4: Observed Transition Frequencies (in $\mathrm{MHz}$ ) for $\mathrm{CF}_{2} \mathrm{CH}^{35} \mathrm{Cl}-\mathrm{H}^{13} \mathrm{CCH}$

\begin{tabular}{|r|r|r|c|c|c|c|c|r|r|}
\hline$J^{\prime}$ & $K_{a}{ }^{\prime}$ & $K_{c}{ }^{\prime}$ & $F^{\prime}$ & $J^{\prime \prime}$ & $K_{a}{ }^{\prime \prime}$ & $K_{c}{ }^{\prime \prime}$ & $F^{\prime \prime}$ & Observed & Obs - Calc \\
\hline 4 & 4 & 0 & 5.5 & 3 & 3 & 1 & 4.5 & 19538.4370 & 0.0009 \\
\hline
\end{tabular}


Table S5: Observed Transition Frequencies (in $\mathrm{MHz}$ ) for $\mathrm{CF}_{2} \mathrm{CH}^{35} \mathrm{Cl}-\mathrm{HC}{ }^{13} \mathrm{CH}$

\begin{tabular}{|c|c|c|c|c|c|c|c|c|c|}
\hline$J^{\prime}$ & $K_{a}{ }^{\prime}$ & $K_{c}{ }^{\prime}$ & $F^{\prime}$ & $J^{\prime \prime}$ & $K_{a}{ }^{\prime \prime}$ & $K_{c}^{\prime \prime}$ & $F^{\prime \prime}$ & Observed & Obs - Calc \\
\hline 3 & 1 & 3 & 4 & 2 & 0 & 2 & 3 & 6055.5479 & 0.0009 \\
\hline 3 & 1 & 3 & 3 & 2 & 0 & 2 & 2 & 6064.0957 & -0.0014 \\
\hline 3 & 1 & 3 & 5 & 2 & 0 & 2 & 4 & 6070.0774 & -0.0009 \\
\hline 3 & 1 & 3 & 2 & 2 & 0 & 2 & 1 & 6078.7204 & -0.0002 \\
\hline 4 & 1 & 4 & 3 & 3 & 1 & 3 & 2 & 6118.7008 & 0.0012 \\
\hline 4 & 1 & 4 & 4 & 3 & 1 & 3 & 3 & 6118.9120 & -0.0008 \\
\hline 4 & 1 & 4 & 6 & 3 & 1 & 3 & 5 & 6119.7177 & -0.0010 \\
\hline 4 & 1 & 4 & 5 & 3 & 1 & 3 & 4 & 6119.7679 & -0.0004 \\
\hline 4 & 0 & 4 & 5 & 3 & 0 & 3 & 4 & 6425.1312 & 0.0005 \\
\hline 4 & 0 & 4 & 4 & 3 & 0 & 3 & 3 & 6426.8446 & 0.0003 \\
\hline 4 & 0 & 4 & 6 & 3 & 0 & 3 & 5 & 6428.5182 & -0.0014 \\
\hline 4 & 0 & 4 & 3 & 3 & 0 & 3 & 2 & 6430.1186 & -0.0048 \\
\hline 4 & 2 & 3 & 3 & 3 & 2 & 2 & 2 & 6659.6660 & 0.0009 \\
\hline 4 & 2 & 3 & 6 & 3 & 2 & 2 & 5 & 6660.3704 & -0.0004 \\
\hline 4 & 2 & 3 & 4 & 3 & 2 & 2 & 3 & 6662.2168 & 0.0004 \\
\hline 4 & 2 & 3 & 5 & 3 & 2 & 2 & 4 & 6663.9273 & 0.0004 \\
\hline 4 & 3 & 2 & 3 & 3 & 3 & 1 & 2 & 6725.2395 & 0.0024 \\
\hline 4 & 3 & 2 & 6 & 3 & 3 & 1 & 5 & 6729.6832 & -0.0012 \\
\hline 4 & 3 & 2 & 4 & 3 & 3 & 1 & 3 & 6734.3164 & -0.0002 \\
\hline 4 & 3 & 1 & 3 & 3 & 3 & 0 & 2 & 6735.8291 & -0.0020 \\
\hline 4 & 3 & 2 & 5 & 3 & 3 & 1 & 4 & 6738.7593 & 0.0012 \\
\hline 4 & 3 & 1 & 6 & 3 & 3 & 0 & 5 & 6741.9517 & 0.0007 \\
\hline 4 & 3 & 1 & 4 & 3 & 3 & 0 & 3 & 6745.6562 & -0.0007 \\
\hline 4 & 3 & 1 & 5 & 3 & 3 & 0 & 4 & 6749.9490 & 0.0011 \\
\hline 4 & 2 & 2 & 6 & 3 & 2 & 1 & 5 & 6915.1189 & -0.0001 \\
\hline 4 & 2 & 2 & 4 & 3 & 2 & 1 & 3 & 6919.9275 & 0.0000 \\
\hline 4 & 2 & 2 & 5 & 3 & 2 & 1 & 4 & 6921.6517 & 0.0003 \\
\hline 5 & 0 & 5 & 4 & 4 & 1 & 4 & 3 & 7026.9161 & 0.0009 \\
\hline 5 & 0 & 5 & 7 & 4 & 1 & 4 & 6 & 7028.2969 & 0.0022 \\
\hline 5 & 0 & 5 & 5 & 4 & 1 & 4 & 4 & 7031.4076 & -0.0014 \\
\hline 5 & 0 & 5 & 6 & 4 & 1 & 4 & 5 & 7033.5924 & 0.0006 \\
\hline 4 & 1 & 3 & 5 & 3 & 1 & 2 & 4 & 7136.4423 & 0.0006 \\
\hline 4 & 1 & 3 & 6 & 3 & 1 & 2 & 5 & 7136.6617 & 0.0009 \\
\hline 4 & 1 & 3 & 4 & 3 & 1 & 2 & 3 & 7138.8332 & -0.0002 \\
\hline 4 & 1 & 3 & 3 & 3 & 1 & 2 & 2 & 7138.9998 & 0.0009 \\
\hline 4 & 1 & 4 & 5 & 3 & 0 & 3 & 4 & 7272.0866 & -0.0009 \\
\hline 4 & 1 & 4 & 4 & 3 & 0 & 3 & 3 & 7276.6762 & -0.0027 \\
\hline 4 & 1 & 4 & 6 & 3 & 0 & 3 & 5 & 7283.7086 & 0.0021 \\
\hline 4 & 1 & 4 & 3 & 3 & 0 & 3 & 2 & 7288.2405 & 0.0002 \\
\hline 5 & 1 & 5 & 5 & 4 & 1 & 4 & 4 & 7608.9232 & -0.0019 \\
\hline 5 & 1 & 5 & 6 & 4 & 1 & 4 & 5 & 7609.3514 & -0.0003 \\
\hline 5 & 1 & 5 & 4 & 4 & 1 & 4 & 3 & 7609.4774 & 0.0007 \\
\hline 5 & 1 & 5 & 7 & 4 & 1 & 4 & 6 & 7609.7346 & 0.0002 \\
\hline
\end{tabular}


Table S5: Observed Transition Frequencies (in $\mathrm{MHz}$ ) for $\mathrm{CF}_{2} \mathrm{CH}^{35} \mathrm{Cl}-\mathrm{HC}{ }^{13} \mathrm{CH}$

\begin{tabular}{|c|c|c|c|c|c|c|c|c|c|}
\hline$J^{\prime}$ & $K_{a}{ }^{\prime}$ & $K_{c}{ }^{\prime}$ & $F^{\prime}$ & $J^{\prime \prime}$ & $K_{a}{ }^{\prime \prime}$ & $K_{c}^{\prime \prime}$ & $F^{\prime \prime}$ & Observed & Obs - Calc \\
\hline 5 & 0 & 5 & 6 & 4 & 0 & 4 & 5 & 7880.5480 & -0.0004 \\
\hline 5 & 0 & 5 & 5 & 4 & 0 & 4 & 4 & 7881.2434 & -0.0001 \\
\hline 5 & 0 & 5 & 7 & 4 & 0 & 4 & 6 & 7883.4806 & -0.0010 \\
\hline 5 & 0 & 5 & 4 & 4 & 0 & 4 & 3 & 7885.0304 & -0.0018 \\
\hline 5 & 2 & 4 & 4 & 4 & 2 & 3 & 3 & 8292.8184 & -0.0019 \\
\hline 5 & 2 & 4 & 7 & 4 & 2 & 3 & 6 & 8293.1665 & -0.0012 \\
\hline 5 & 2 & 4 & 6 & 4 & 2 & 3 & 5 & 8294.0717 & 0.0001 \\
\hline 5 & 2 & 4 & 5 & 4 & 2 & 3 & 4 & 8294.3010 & -0.0001 \\
\hline 5 & 3 & 3 & 4 & 4 & 3 & 2 & 3 & 8426.7251 & 0.0008 \\
\hline 5 & 3 & 3 & 7 & 4 & 3 & 2 & 6 & 8428.1664 & -0.0011 \\
\hline 5 & 3 & 3 & 5 & 4 & 3 & 2 & 4 & 8431.3842 & -0.0001 \\
\hline 5 & 3 & 3 & 6 & 4 & 3 & 2 & 5 & 8432.8398 & -0.0002 \\
\hline 5 & 1 & 5 & 6 & 4 & 0 & 4 & 5 & 8456.3089 & 0.0005 \\
\hline 5 & 1 & 5 & 5 & 4 & 0 & 4 & 4 & 8458.7596 & 0.0000 \\
\hline 5 & 3 & 2 & 4 & 4 & 3 & 1 & 3 & 8463.5362 & -0.0002 \\
\hline 5 & 3 & 2 & 7 & 4 & 3 & 1 & 6 & 8463.5674 & -0.0006 \\
\hline 5 & 1 & 5 & 7 & 4 & 0 & 4 & 6 & 8464.9243 & 0.0030 \\
\hline 5 & 1 & 5 & 4 & 4 & 0 & 4 & 3 & 8467.5935 & -0.0002 \\
\hline 5 & 3 & 2 & 5 & 4 & 3 & 1 & 4 & 8468.4404 & -0.0002 \\
\hline 5 & 3 & 2 & 6 & 4 & 3 & 1 & 5 & 8470.3148 & -0.0004 \\
\hline 2 & 2 & 1 & 3 & 1 & 1 & 0 & 2 & 8639.6165 & 0.0013 \\
\hline 2 & 2 & 1 & 2 & 1 & 1 & 0 & 2 & 8646.2090 & 0.0000 \\
\hline 2 & 2 & 1 & 4 & 1 & 1 & 0 & 3 & 8657.8479 & 0.0016 \\
\hline 2 & 2 & 1 & 2 & 1 & 1 & 0 & 1 & 8662.5703 & 0.0023 \\
\hline 6 & 0 & 6 & 5 & 5 & 1 & 5 & 4 & 8709.9147 & -0.0001 \\
\hline 6 & 0 & 6 & 8 & 5 & 1 & 5 & 7 & 8710.8236 & 0.0000 \\
\hline 6 & 0 & 6 & 6 & 5 & 1 & 5 & 5 & 8712.4529 & -0.0009 \\
\hline 6 & 0 & 6 & 7 & 5 & 1 & 5 & 6 & 8713.4597 & -0.0008 \\
\hline 5 & 2 & 3 & 4 & 4 & 2 & 2 & 3 & 8761.4125 & 0.0012 \\
\hline 5 & 2 & 3 & 7 & 4 & 2 & 2 & 6 & 8762.3227 & 0.0004 \\
\hline 5 & 2 & 3 & 6 & 4 & 2 & 2 & 5 & 8765.1261 & 0.0008 \\
\hline 5 & 2 & 3 & 5 & 4 & 2 & 2 & 4 & 8766.0788 & 0.0016 \\
\hline 5 & 1 & 4 & 6 & 4 & 1 & 3 & 5 & 8856.5318 & 0.0005 \\
\hline 5 & 1 & 4 & 7 & 4 & 1 & 3 & 6 & 8857.6638 & 0.0001 \\
\hline 5 & 1 & 4 & 5 & 4 & 1 & 3 & 4 & 8858.2081 & -0.0001 \\
\hline 5 & 1 & 4 & 4 & 4 & 1 & 3 & 3 & 8859.3212 & -0.0011 \\
\hline 2 & 2 & 0 & 3 & 1 & 1 & 1 & 3 & 8928.9559 & -0.0044 \\
\hline 2 & 2 & 0 & 4 & 1 & 1 & 1 & 3 & 8936.1200 & -0.0015 \\
\hline 6 & 1 & 6 & 6 & 5 & 1 & 5 & 5 & 9079.9743 & -0.0014 \\
\hline 6 & 1 & 6 & 7 & 5 & 1 & 5 & 6 & 9080.1572 & -0.0029 \\
\hline 6 & 1 & 6 & 5 & 5 & 1 & 5 & 4 & 9080.6442 & -0.0006 \\
\hline 6 & 1 & 6 & 8 & 5 & 1 & 5 & 7 & 9080.8468 & 0.0002 \\
\hline 6 & 0 & 6 & 7 & 5 & 0 & 5 & 6 & 9289.2186 & -0.0017 \\
\hline
\end{tabular}


Table S5: Observed Transition Frequencies (in $\mathrm{MHz}$ ) for $\mathrm{CF}_{2} \mathrm{CH}^{35} \mathrm{Cl}-\mathrm{HC}{ }^{13} \mathrm{CH}$

\begin{tabular}{|c|c|c|c|c|c|c|c|c|c|}
\hline$J^{\prime}$ & $K_{a}^{\prime}$ & $K_{c}{ }^{\prime}$ & $F^{\prime}$ & $J^{\prime \prime}$ & $K_{a}^{\prime \prime}$ & $K_{c}{ }^{\prime \prime}$ & $F^{\prime \prime}$ & Observed & Obs - Calc \\
\hline 6 & 0 & 6 & 6 & 5 & 0 & 5 & 5 & 9289.9691 & -0.0009 \\
\hline 6 & 0 & 6 & 8 & 5 & 0 & 5 & 7 & 9292.2629 & -0.0005 \\
\hline 6 & 0 & 6 & 5 & 5 & 0 & 5 & 4 & 9292.4753 & -0.0009 \\
\hline 6 & 1 & 6 & 7 & 5 & 0 & 5 & 6 & 9655.9195 & -0.0005 \\
\hline 6 & 1 & 6 & 6 & 5 & 0 & 5 & 5 & 9657.4926 & 0.0008 \\
\hline 6 & 1 & 6 & 8 & 5 & 0 & 5 & 7 & 9662.2855 & -0.0009 \\
\hline 6 & 1 & 6 & 5 & 5 & 0 & 5 & 4 & 9663.2027 & -0.0035 \\
\hline 6 & 2 & 5 & 8 & 5 & 2 & 4 & 7 & 9902.7950 & 0.0013 \\
\hline 6 & 2 & 5 & 7 & 5 & 2 & 4 & 6 & 9902.9226 & 0.0012 \\
\hline 6 & 2 & 5 & 6 & 5 & 2 & 4 & 5 & 9903.1063 & 0.0006 \\
\hline 3 & 2 & 2 & 4 & 2 & 1 & 1 & 3 & 10054.0124 & -0.0001 \\
\hline 3 & 2 & 2 & 3 & 2 & 1 & 1 & 2 & 10067.2765 & -0.0020 \\
\hline 3 & 2 & 2 & 5 & 2 & 1 & 1 & 4 & 10072.3741 & 0.0035 \\
\hline 3 & 2 & 2 & 2 & 2 & 1 & 1 & 1 & 10085.3060 & -0.0010 \\
\hline 6 & 4 & 3 & 5 & 5 & 4 & 2 & 4 & 10112.6920 & 0.0015 \\
\hline 6 & 4 & 3 & 8 & 5 & 4 & 2 & 7 & 10114.0296 & -0.0025 \\
\hline 6 & 4 & 2 & 5 & 5 & 4 & 1 & 4 & 10116.2102 & 0.0021 \\
\hline 6 & 4 & 3 & 6 & 5 & 4 & 2 & 5 & 10117.5100 & -0.0003 \\
\hline 6 & 4 & 2 & 8 & 5 & 4 & 1 & 7 & 10117.5730 & -0.0030 \\
\hline 6 & 4 & 3 & 7 & 5 & 4 & 2 & 6 & 10118.8284 & -0.0001 \\
\hline 6 & 4 & 2 & 6 & 5 & 4 & 1 & 5 & 10121.1038 & -0.0002 \\
\hline 6 & 4 & 2 & 7 & 5 & 4 & 1 & 6 & 10122.4334 & 0.0005 \\
\hline 6 & 3 & 4 & 5 & 5 & 3 & 3 & 4 & 10127.1581 & 0.0001 \\
\hline 6 & 3 & 4 & 8 & 5 & 3 & 3 & 7 & 10127.7962 & 0.0005 \\
\hline 6 & 3 & 4 & 6 & 5 & 3 & 3 & 5 & 10130.0143 & 0.0014 \\
\hline 6 & 3 & 4 & 7 & 5 & 3 & 3 & 6 & 10130.3703 & -0.0020 \\
\hline 6 & 3 & 3 & 5 & 5 & 3 & 2 & 4 & 10222.8251 & 0.0002 \\
\hline 6 & 3 & 3 & 8 & 5 & 3 & 2 & 7 & 10223.5673 & -0.0011 \\
\hline 6 & 3 & 3 & 6 & 5 & 3 & 2 & 5 & 10226.6931 & 0.0004 \\
\hline 6 & 3 & 3 & 7 & 5 & 3 & 2 & 6 & 10227.4167 & -0.0015 \\
\hline 7 & 0 & 7 & 6 & 6 & 1 & 6 & 5 & 10311.1583 & -0.0005 \\
\hline 7 & 0 & 7 & 9 & 6 & 1 & 6 & 8 & 10311.6299 & 0.0005 \\
\hline 7 & 0 & 7 & 7 & 6 & 1 & 6 & 6 & 10312.2323 & -0.0006 \\
\hline 7 & 0 & 7 & 8 & 6 & 1 & 6 & 7 & 10312.7606 & -0.0013 \\
\hline 6 & 1 & 5 & 7 & 5 & 1 & 4 & 6 & 10525.0657 & -0.0004 \\
\hline 6 & 1 & 5 & 6 & 5 & 1 & 4 & 5 & 10526.3619 & 0.0002 \\
\hline 6 & 1 & 5 & 8 & 5 & 1 & 4 & 7 & 10526.9202 & -0.0001 \\
\hline 6 & 1 & 5 & 5 & 5 & 1 & 4 & 4 & 10528.1880 & 0.0000 \\
\hline 7 & 1 & 7 & 7 & 6 & 1 & 6 & 6 & 10534.3445 & -0.0010 \\
\hline 7 & 1 & 7 & 8 & 6 & 1 & 6 & 7 & 10534.4672 & -0.0012 \\
\hline 7 & 1 & 7 & 6 & 6 & 1 & 6 & 5 & 10535.0956 & -0.0004 \\
\hline 7 & 1 & 7 & 9 & 6 & 1 & 6 & 8 & 10535.2239 & -0.0001 \\
\hline 6 & 2 & 4 & 8 & 5 & 2 & 3 & 7 & 10631.2336 & 0.0012 \\
\hline
\end{tabular}


Table S5: Observed Transition Frequencies (in $\mathrm{MHz}$ ) for $\mathrm{CF}_{2} \mathrm{CH}^{35} \mathrm{Cl}-\mathrm{HC}{ }^{13} \mathrm{CH}$

\begin{tabular}{|c|c|c|c|c|c|c|c|c|c|}
\hline$J^{\prime}$ & $K_{a}^{\prime}$ & $K_{c}{ }^{\prime}$ & $F^{\prime}$ & $J^{\prime \prime}$ & $K_{a}^{\prime \prime}$ & $K_{c}{ }^{\prime \prime}$ & $F^{\prime \prime}$ & Observed & Obs - Calc \\
\hline 6 & 2 & 4 & 5 & 5 & 2 & 3 & 4 & 10631.7154 & 0.0009 \\
\hline 6 & 2 & 4 & 6 & 5 & 2 & 3 & 5 & 10634.3243 & 0.0013 \\
\hline 6 & 2 & 4 & 7 & 5 & 2 & 3 & 6 & 10635.6853 & -0.0009 \\
\hline 7 & 0 & 7 & 8 & 6 & 0 & 6 & 7 & 10679.4605 & -0.0011 \\
\hline 7 & 0 & 7 & 7 & 6 & 0 & 6 & 6 & 10679.7539 & -0.0008 \\
\hline 7 & 0 & 7 & 9 & 6 & 0 & 6 & 8 & 10681.6517 & -0.0008 \\
\hline 7 & 0 & 7 & 6 & 6 & 0 & 6 & 5 & 10681.8875 & -0.0014 \\
\hline 7 & 1 & 7 & 8 & 6 & 0 & 6 & 7 & 10901.1684 & 0.0003 \\
\hline 7 & 1 & 7 & 7 & 6 & 0 & 6 & 6 & 10901.8670 & -0.0003 \\
\hline 7 & 1 & 7 & 9 & 6 & 0 & 6 & 8 & 10905.2468 & -0.0002 \\
\hline 7 & 1 & 7 & 6 & 6 & 0 & 6 & 5 & 10905.8262 & 0.0001 \\
\hline 3 & 2 & 1 & 2 & 2 & 1 & 2 & 1 & 10959.6610 & 0.0007 \\
\hline 3 & 2 & 1 & 5 & 2 & 1 & 2 & 4 & 10968.4534 & -0.0017 \\
\hline 3 & 2 & 1 & 3 & 2 & 1 & 2 & 2 & 10973.5421 & -0.0019 \\
\hline 3 & 2 & 1 & 4 & 2 & 1 & 2 & 3 & 10982.3546 & -0.0012 \\
\hline 4 & 2 & 3 & 5 & 3 & 1 & 2 & 4 & 11337.5525 & -0.0002 \\
\hline 4 & 2 & 3 & 4 & 3 & 1 & 2 & 3 & 11345.3351 & -0.0007 \\
\hline 4 & 2 & 3 & 6 & 3 & 1 & 2 & 5 & 11353.9978 & 0.0033 \\
\hline 4 & 2 & 3 & 3 & 3 & 1 & 2 & 2 & 11362.4224 & -0.0023 \\
\hline 7 & 2 & 6 & 8 & 6 & 2 & 5 & 7 & 11486.8706 & 0.0000 \\
\hline 7 & 2 & 6 & 7 & 6 & 2 & 5 & 6 & 11487.1201 & -0.0005 \\
\hline 7 & 2 & 6 & 9 & 6 & 2 & 5 & 8 & 11487.3034 & 0.0002 \\
\hline 7 & 2 & 6 & 6 & 6 & 2 & 5 & 5 & 11487.5044 & 0.0008 \\
\hline 7 & 3 & 5 & 9 & 6 & 3 & 4 & 8 & 11822.7840 & -0.0017 \\
\hline 7 & 3 & 5 & 6 & 6 & 3 & 4 & 5 & 11822.8002 & 0.0034 \\
\hline 7 & 4 & 4 & 6 & 6 & 4 & 3 & 5 & 11824.0594 & -0.0001 \\
\hline 7 & 3 & 5 & 7 & 6 & 3 & 4 & 6 & 11824.1974 & 0.0001 \\
\hline 7 & 3 & 5 & 8 & 6 & 3 & 4 & 7 & 11824.5410 & 0.0010 \\
\hline 7 & 4 & 4 & 9 & 6 & 4 & 3 & 8 & 11824.6776 & -0.0008 \\
\hline 7 & 4 & 4 & 7 & 6 & 4 & 3 & 6 & 11827.2122 & -0.0012 \\
\hline 7 & 4 & 4 & 8 & 6 & 4 & 3 & 7 & 11827.8602 & -0.0012 \\
\hline 7 & 4 & 3 & 6 & 6 & 4 & 2 & 5 & 11835.6574 & 0.0012 \\
\hline 7 & 4 & 3 & 9 & 6 & 4 & 2 & 8 & 11836.2985 & -0.0015 \\
\hline 7 & 4 & 3 & 7 & 6 & 4 & 2 & 6 & 11838.9653 & -0.0005 \\
\hline 7 & 4 & 3 & 8 & 6 & 4 & 2 & 7 & 11839.6470 & -0.0012 \\
\hline 8 & 0 & 8 & 7 & 7 & 1 & 7 & 6 & 11845.8816 & 0.0005 \\
\hline 8 & 0 & 8 & 8 & 7 & 1 & 7 & 7 & 11846.1210 & -0.0029 \\
\hline 8 & 0 & 8 & 10 & 7 & 1 & 7 & 9 & 11846.1366 & 0.0035 \\
\hline 8 & 0 & 8 & 9 & 7 & 1 & 7 & 8 & 11846.4177 & 0.0012 \\
\hline 8 & 1 & 8 & 8 & 7 & 1 & 7 & 7 & 11975.2589 & 0.0000 \\
\hline 8 & 1 & 8 & 9 & 7 & 1 & 7 & 8 & 11975.3532 & 0.0009 \\
\hline 8 & 1 & 8 & 7 & 7 & 1 & 7 & 6 & 11976.0038 & 0.0008 \\
\hline 8 & 1 & 8 & 10 & 7 & 1 & 7 & 9 & 11976.0979 & 0.0001 \\
\hline
\end{tabular}


Table S5: Observed Transition Frequencies (in $\mathrm{MHz}$ ) for $\mathrm{CF}_{2} \mathrm{CH}^{35} \mathrm{Cl}-\mathrm{HC}{ }^{13} \mathrm{CH}$

\begin{tabular}{|c|c|c|c|c|c|c|c|c|c|}
\hline$J^{\prime}$ & $K_{a}^{\prime}$ & $K_{c}{ }^{\prime}$ & $F^{\prime}$ & $J^{\prime \prime}$ & $K_{a}^{\prime \prime}$ & $K_{c}{ }^{\prime \prime}$ & $F^{\prime \prime}$ & Observed & Obs - Calc \\
\hline 7 & 3 & 4 & 6 & 6 & 3 & 3 & 5 & 12027.7056 & 0.0006 \\
\hline 7 & 3 & 4 & 9 & 6 & 3 & 3 & 8 & 12028.1492 & 0.0009 \\
\hline 7 & 3 & 4 & 7 & 6 & 3 & 3 & 6 & 12031.0394 & 0.0003 \\
\hline 7 & 3 & 4 & 8 & 6 & 3 & 3 & 7 & 12031.4444 & 0.0015 \\
\hline 8 & 0 & 8 & 9 & 7 & 0 & 7 & 8 & 12068.1250 & 0.0019 \\
\hline 8 & 0 & 8 & 8 & 7 & 0 & 7 & 7 & 12068.2359 & -0.0006 \\
\hline 8 & 0 & 8 & 10 & 7 & 0 & 7 & 9 & 12069.7276 & -0.0002 \\
\hline 8 & 0 & 8 & 7 & 7 & 0 & 7 & 6 & 12069.8187 & 0.0003 \\
\hline 7 & 1 & 6 & 8 & 6 & 1 & 5 & 7 & 12126.1763 & 0.0005 \\
\hline 7 & 1 & 6 & 7 & 6 & 1 & 5 & 6 & 12127.3316 & 0.0004 \\
\hline 7 & 1 & 6 & 9 & 6 & 1 & 5 & 8 & 12128.7536 & 0.0000 \\
\hline 7 & 1 & 6 & 6 & 6 & 1 & 5 & 5 & 12129.6627 & -0.0002 \\
\hline 8 & 1 & 8 & 9 & 7 & 0 & 7 & 8 & 12197.0586 & -0.0003 \\
\hline 8 & 1 & 8 & 8 & 7 & 0 & 7 & 7 & 12197.3709 & -0.0006 \\
\hline 8 & 1 & 8 & 10 & 7 & 0 & 7 & 9 & 12199.6934 & 0.0010 \\
\hline 8 & 1 & 8 & 7 & 7 & 0 & 7 & 6 & 12199.9413 & 0.0011 \\
\hline 7 & 2 & 5 & 9 & 6 & 2 & 4 & 8 & 12488.7331 & 0.0013 \\
\hline 7 & 2 & 5 & 6 & 6 & 2 & 4 & 5 & 12489.0121 & 0.0009 \\
\hline 7 & 2 & 5 & 8 & 6 & 2 & 4 & 7 & 12490.3211 & 0.0017 \\
\hline 7 & 2 & 5 & 7 & 6 & 2 & 4 & 6 & 12490.6120 & 0.0010 \\
\hline 5 & 2 & 4 & 6 & 4 & 1 & 3 & 5 & 12495.1863 & 0.0037 \\
\hline 5 & 2 & 4 & 5 & 4 & 1 & 3 & 4 & 12500.8076 & 0.0042 \\
\hline 5 & 2 & 4 & 7 & 4 & 1 & 3 & 6 & 12510.5064 & 0.0050 \\
\hline 5 & 2 & 4 & 4 & 4 & 1 & 3 & 3 & 12516.2459 & -0.0002 \\
\hline 8 & 2 & 7 & 9 & 7 & 2 & 6 & 8 & 13043.8101 & 0.0012 \\
\hline 8 & 2 & 7 & 8 & 7 & 2 & 6 & 7 & 13044.0562 & 0.0000 \\
\hline 8 & 2 & 7 & 10 & 7 & 2 & 6 & 9 & 13044.5615 & 0.0009 \\
\hline 8 & 2 & 7 & 7 & 7 & 2 & 6 & 6 & 13044.7870 & 0.0012 \\
\hline 4 & 2 & 2 & 6 & 3 & 1 & 3 & 5 & 13273.1381 & -0.0029 \\
\hline 4 & 2 & 2 & 4 & 3 & 1 & 3 & 3 & 13284.4190 & 0.0020 \\
\hline 4 & 2 & 2 & 5 & 3 & 1 & 3 & 4 & 13291.8758 & -0.0009 \\
\hline 9 & 0 & 9 & 9 & 8 & 1 & 8 & 8 & 13333.2263 & -0.0012 \\
\hline 9 & 0 & 9 & 8 & 8 & 1 & 8 & 7 & \multirow{2}{*}{13333.4069} & \multirow{2}{*}{0.0004} \\
\hline 9 & 0 & 9 & 10 & 8 & 1 & 8 & 9 & & \\
\hline 9 & 0 & 9 & 11 & 8 & 1 & 8 & 10 & 13333.5566 & 0.0007 \\
\hline 9 & 0 & 9 & 10 & 8 & 0 & 8 & 9 & 13462.3427 & 0.0003 \\
\hline 9 & 0 & 9 & 9 & 8 & 0 & 8 & 8 & 13462.3607 & -0.0018 \\
\hline 9 & 0 & 9 & 11 & 8 & 0 & 8 & 10 & 13463.5199 & -0.0007 \\
\hline 9 & 0 & 9 & 8 & 8 & 0 & 8 & 7 & 13463.5292 & 0.0011 \\
\hline 8 & 3 & 6 & 10 & 7 & 3 & 5 & 9 & 13507.6201 & 0.0010 \\
\hline 8 & 3 & 6 & 7 & 7 & 3 & 5 & 6 & 13507.6391 & 0.0042 \\
\hline 8 & 3 & 6 & 9 & 7 & 3 & 5 & 8 & 13508.5127 & -0.0005 \\
\hline 8 & 3 & 6 & 8 & 7 & 3 & 5 & 7 & 13508.5392 & 0.0003 \\
\hline
\end{tabular}


Table S5: Observed Transition Frequencies (in $\mathrm{MHz}$ ) for $\mathrm{CF}_{2} \mathrm{CH}^{35} \mathrm{Cl}-\mathrm{HC}{ }^{13} \mathrm{CH}$

\begin{tabular}{|c|c|c|c|c|c|c|c|c|c|}
\hline$J^{\prime}$ & $K_{a}^{\prime}$ & $K_{c}{ }^{\prime}$ & $F^{\prime}$ & $J^{\prime \prime}$ & $K_{a}^{\prime \prime}$ & $K_{c}{ }^{\prime \prime}$ & $F^{\prime \prime}$ & Observed & Obs - Calc \\
\hline 9 & 1 & 9 & 10 & 8 & 0 & 8 & 9 & 13535.1772 & 0.0004 \\
\hline 9 & 1 & 9 & 9 & 8 & 0 & 8 & 8 & 13535.2947 & 0.0000 \\
\hline 9 & 1 & 9 & 11 & 8 & 0 & 8 & 10 & 13536.9008 & 0.0010 \\
\hline 9 & 1 & 9 & 8 & 8 & 0 & 8 & 7 & 13536.9767 & 0.0003 \\
\hline 6 & 2 & 5 & 8 & 5 & 1 & 4 & 7 & 13555.6352 & 0.0037 \\
\hline 6 & 2 & 5 & 5 & 5 & 1 & 4 & 4 & 13559.8832 & 0.0007 \\
\hline 8 & 1 & 7 & 9 & 7 & 1 & 6 & 8 & 13649.7951 & 0.0007 \\
\hline 8 & 1 & 7 & 8 & 7 & 1 & 6 & 7 & 13650.5098 & 0.0006 \\
\hline 8 & 1 & 7 & 10 & 7 & 1 & 6 & 9 & 13652.5481 & 0.0005 \\
\hline 8 & 1 & 7 & 7 & 7 & 1 & 6 & 6 & 13653.6433 & 0.0005 \\
\hline 8 & 3 & 5 & 7 & 7 & 3 & 4 & 6 & 13885.6732 & 0.0019 \\
\hline 8 & 3 & 5 & 10 & 7 & 3 & 4 & 9 & 13885.9241 & 0.0022 \\
\hline 8 & 3 & 5 & 8 & 7 & 3 & 4 & 7 & 13888.7107 & 0.0001 \\
\hline 8 & 3 & 5 & 9 & 7 & 3 & 4 & 8 & 13889.0046 & 0.0002 \\
\hline 3 & 3 & 1 & 4 & 2 & 2 & 0 & 3 & 14065.8557 & 0.0014 \\
\hline 3 & 3 & 1 & 5 & 2 & 2 & 0 & 4 & 14069.5939 & 0.0011 \\
\hline 3 & 3 & 0 & 3 & 2 & 2 & 1 & 2 & 14094.8406 & 0.0012 \\
\hline 3 & 3 & 0 & 4 & 2 & 2 & 1 & 3 & 14096.3877 & 0.0003 \\
\hline 3 & 3 & 0 & 5 & 2 & 2 & 1 & 4 & 14098.0045 & 0.0003 \\
\hline 8 & 2 & 6 & 10 & 7 & 2 & 5 & 9 & 14308.5475 & -0.0004 \\
\hline 8 & 2 & 6 & 7 & 7 & 2 & 5 & 6 & 14308.9485 & 0.0012 \\
\hline 8 & 2 & 6 & 9 & 7 & 2 & 5 & 8 & 14309.0692 & -0.0013 \\
\hline 8 & 2 & 6 & 8 & 7 & 2 & 5 & 7 & 14309.4781 & -0.0006 \\
\hline 9 & 2 & 8 & 10 & 8 & 2 & 7 & 9 & 14573.1525 & 0.0000 \\
\hline 9 & 2 & 8 & 9 & 8 & 2 & 7 & 8 & 14573.3737 & 0.0008 \\
\hline 9 & 2 & 8 & 11 & 8 & 2 & 7 & 10 & 14574.1048 & 0.0012 \\
\hline 9 & 2 & 8 & 8 & 8 & 2 & 7 & 7 & 14574.3123 & 0.0008 \\
\hline 10 & 0 & 10 & 10 & 9 & 1 & 9 & 9 & 14789.8356 & -0.0008 \\
\hline 10 & 0 & 10 & 11 & 9 & 1 & 9 & 10 & 14789.9605 & 0.0002 \\
\hline 10 & 0 & 10 & 9 & 9 & 1 & 9 & 8 & 14790.1971 & -0.0026 \\
\hline 10 & 0 & 10 & 12 & 9 & 1 & 9 & 11 & 14790.3038 & 0.0012 \\
\hline 10 & 1 & 10 & 10 & 9 & 1 & 9 & 9 & 14830.1246 & 0.0009 \\
\hline 10 & 1 & 10 & 11 & 9 & 1 & 9 & 10 & 14830.1985 & -0.0008 \\
\hline 10 & 1 & 10 & 9 & 9 & 1 & 9 & 8 & 14830.7522 & 0.0016 \\
\hline 10 & 1 & 10 & 12 & 9 & 1 & 9 & 11 & 14830.8248 & 0.0003 \\
\hline 10 & 0 & 10 & 9 & 9 & 0 & 9 & 8 & 14863.6490 & 0.0011 \\
\hline 10 & 0 & 10 & 12 & 9 & 0 & 9 & 11 & 14863.6828 & 0.0010 \\
\hline 9 & 1 & 8 & 10 & 8 & 1 & 7 & 9 & 15097.9609 & 0.0007 \\
\hline 9 & 1 & 8 & 9 & 8 & 1 & 7 & 8 & 15098.6897 & -0.0025 \\
\hline 9 & 1 & 8 & 11 & 8 & 1 & 7 & 10 & 15101.0790 & -0.0011 \\
\hline 9 & 1 & 8 & 8 & 8 & 1 & 7 & 7 & 15101.7673 & 0.0002 \\
\hline 9 & 3 & 7 & 11 & 8 & 3 & 6 & 10 & 15175.0565 & 0.0002 \\
\hline 9 & 3 & 7 & 10 & 8 & 3 & 6 & 9 & 15175.4251 & 0.0008 \\
\hline
\end{tabular}


Table S5: Observed Transition Frequencies (in $\mathrm{MHz}$ ) for $\mathrm{CF}_{2} \mathrm{CH}^{35} \mathrm{Cl}-\mathrm{HC}{ }^{13} \mathrm{CH}$

\begin{tabular}{|r|r|c|c|c|c|c|c|r|r|}
\hline$J^{\prime}$ & $K_{a}^{\prime}$ & $K_{c}{ }^{\prime}$ & $F^{\prime}$ & $J^{\prime \prime}$ & $K_{a}^{\prime \prime}$ & $K_{c}{ }^{\prime \prime}$ & $F^{\prime \prime}$ & Observed & Obs - Calc \\
\hline 9 & 3 & 7 & 9 & 8 & 3 & 6 & 8 & 15175.5176 & -0.0025 \\
\hline 4 & 3 & 2 & 5 & 3 & 2 & 1 & 4 & 15676.7588 & -0.0010 \\
\hline 4 & 3 & 2 & 4 & 3 & 2 & 1 & 3 & 15679.6966 & -0.0011 \\
\hline 4 & 3 & 2 & 6 & 3 & 2 & 1 & 5 & 15683.4597 & -0.0005 \\
\hline 4 & 3 & 2 & 3 & 3 & 2 & 1 & 2 & 15686.3281 & 0.0005 \\
\hline 9 & 3 & 6 & 8 & 8 & 3 & 5 & 7 & 15789.8735 & 0.0029 \\
\hline 9 & 3 & 6 & 11 & 8 & 3 & 5 & 10 & 15790.0426 & 0.0007 \\
\hline 9 & 3 & 6 & 9 & 8 & 3 & 5 & 8 & 15792.6232 & -0.0025 \\
\hline 9 & 3 & 6 & 10 & 8 & 3 & 5 & 9 & 15792.7842 & -0.0023 \\
\hline 4 & 3 & 1 & 5 & 3 & 2 & 2 & 4 & 15827.2602 & 0.0002 \\
\hline 4 & 3 & 1 & 4 & 3 & 2 & 2 & 3 & 15827.8412 & -0.0016 \\
\hline 4 & 3 & 1 & 3 & 3 & 2 & 2 & 2 & 15829.0229 & -0.0004 \\
\hline 4 & 3 & 1 & 6 & 3 & 2 & 2 & 5 & 15829.9942 & 0.0005 \\
\hline 9 & 2 & 7 & 10 & 8 & 2 & 6 & 9 & 16072.6873 & 0.0006 \\
\hline 9 & 2 & 7 & 11 & 8 & 2 & 6 & 10 & 16073.0449 & -0.0009 \\
\hline 9 & 2 & 7 & 9 & 8 & 2 & 6 & 8 & 16073.1488 & -0.0012 \\
\hline 9 & 2 & 7 & 8 & 8 & 2 & 6 & 7 & 16073.4909 & -0.0028 \\
\hline 10 & 1 & 9 & 11 & 9 & 1 & 8 & 10 & 16491.0324 & -0.0009 \\
\hline 10 & 1 & 9 & 10 & 9 & 1 & 8 & 9 & 16491.5895 & -0.0006 \\
\hline 10 & 1 & 9 & 12 & 9 & 1 & 8 & 11 & 16493.9798 & -0.0018 \\
\hline 10 & 1 & 9 & 9 & 9 & 1 & 8 & 8 & 16494.5246 & -0.0020 \\
\hline 5 & 3 & 3 & 6 & 4 & 2 & 2 & 5 & 17187.9480 & -0.0005 \\
\hline 5 & 3 & 3 & 5 & 4 & 2 & 2 & 4 & 17191.1524 & -0.0022 \\
\hline 5 & 3 & 3 & 7 & 4 & 2 & 2 & 6 & 17196.5086 & -0.0002 \\
\hline 5 & 3 & 3 & 4 & 4 & 2 & 2 & 3 & 17199.6908 & -0.0020 \\
\hline 5 & 3 & 2 & 4 & 4 & 2 & 3 & 3 & 17632.8952 & 0.0007 \\
\hline 5 & 3 & 2 & 7 & 4 & 2 & 3 & 6 & 17633.1902 & -0.0007 \\
\hline 5 & 3 & 2 & 6 & 4 & 2 & 3 & 5 & 17633.6466 & -0.0017 \\
\hline 5 & 3 & 2 & 5 & 4 & 2 & 3 & 4 & 17634.0660 & -0.0010 \\
\hline 4 & 4 & 1 & 4 & 3 & 3 & 0 & 3 & 19377.1315 & 0.0017 \\
\hline 4 & 4 & 1 & 5 & 3 & 3 & 0 & 4 & 19377.8267 & -0.0006 \\
\hline 4 & 4 & 1 & 3 & 3 & 3 & 0 & 2 & 19378.7196 & 0.0016 \\
\hline 4 & 4 & 0 & 4 & 3 & 3 & 1 & 3 & 19379.0650 & -0.0013 \\
\hline 4 & 4 & 1 & 6 & 3 & 3 & 0 & 5 & 19379.4223 & -0.0003 \\
\hline 4 & 4 & 0 & 5 & 3 & 3 & 1 & 4 & 19379.8123 & -0.0005 \\
\hline 4 & 4 & 0 & 6 & 3 & 3 & 1 & 5 & 19381.3023 & 0.0011 \\
\hline
\end{tabular}

